\title{
Modulation of the ROCK pathway in models of Parkinson's disease
}

\author{
PhD Thesis \\ for the award of the degree \\ "Doctor rerum naturalium" \\ within the doctoral basic program Biology \\ of the Georg-August University School of Science
}

submitted by

Kim Ann Saal

born in

Bad Harzburg (Germany)

Göttingen, December 2014 


\section{Declaration}

I hereby declare that the thesis:

"Modulation of the ROCK pathway in models of Parkinson's disease"

has been written independently and with no other sources and aids than quoted.

Kim Ann Saal

Göttingen, December 2014 
Thesis committee:

Prof. Dr. Paul Lingor (Department of Neurology, University Medicine Göttingen)

Prof. Dr. Mathias Bähr (Department of Neurology, University Medicine Göttingen)

Prof. Dr. Martin Göpfert (Department of Cellular Neurobiology, Schwann-Schleiden Research Centre, Georg-August-University Göttingen)

\section{Examination committee:}

1. Referee: Prof. Dr. Mathias Bähr (Department of Neurology, University Medicine Göttingen)

2. Referee: Prof. Dr. Martin Göpfert (Department of Cellular Neurobiology, SchwannSchleiden Research Centre, Georg-August-University Göttingen)

\section{Extended examination committee:}

Prof. Dr. Silvio Rizzoli (Department of Neuro-and Sensoryphysiology, University Medicine Göttingen)

Prof. Dr. Sigrid Hoyer-Fender (Department of Developmental Biology, Johann-FriedrichBlumenbach-Institute of Zoology und Anthropology, Georg-August-University Göttingen)

Dr. Sebastian Kügler (Department of Neurology, University Medicine Göttingen)

Prof. Dr. Gerhard Braus (Department of Molecular Microbiology and Genetics, Institute for Microbiology and Genetics, Georg-August-University Göttingen)

Day of disputation: $\quad 16^{\text {th }}$ of January 2015 


\section{Table of contents}

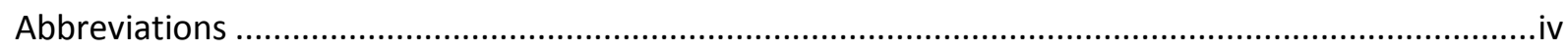

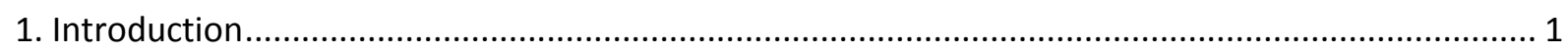

1.1. Preface

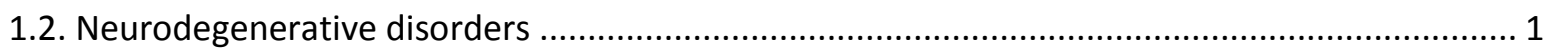

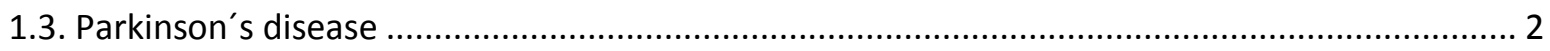

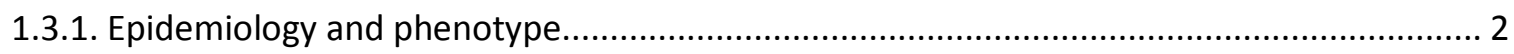

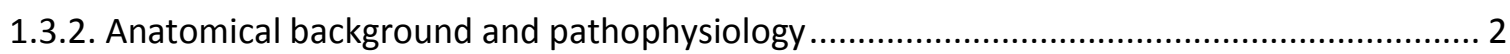

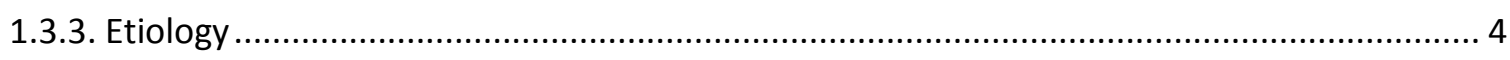

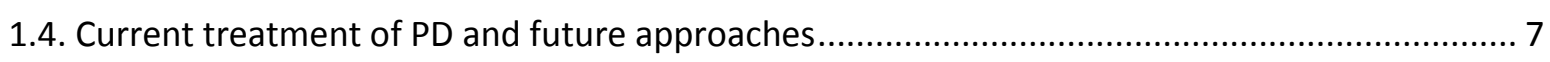

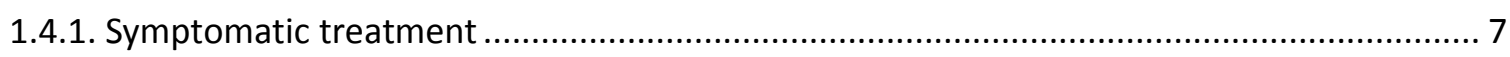

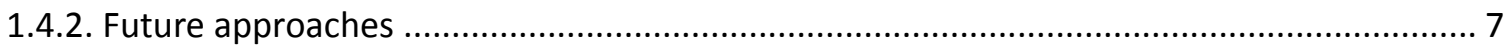

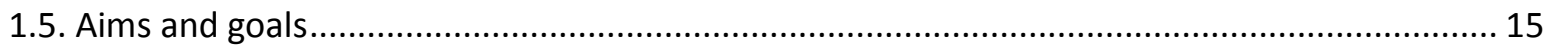

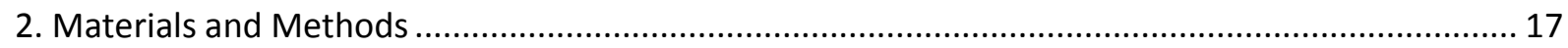

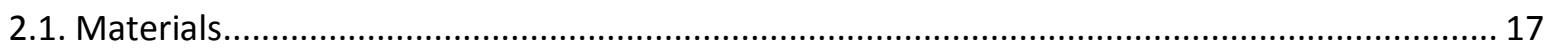

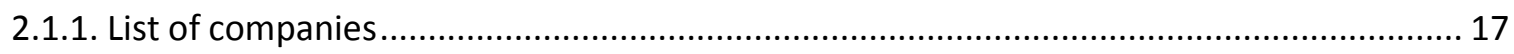

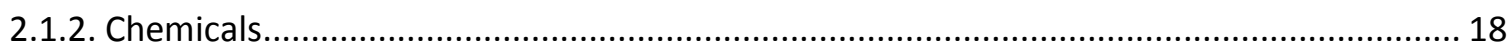

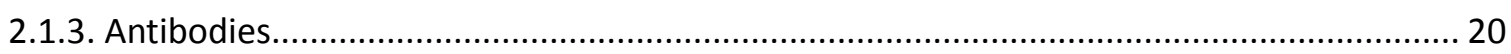

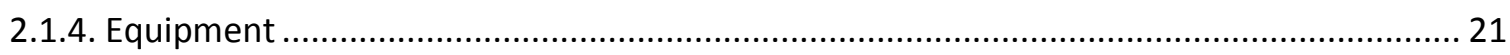

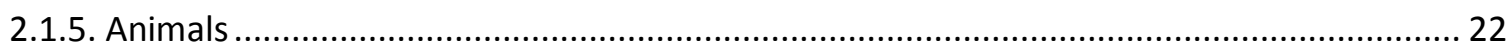

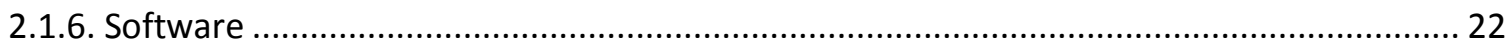

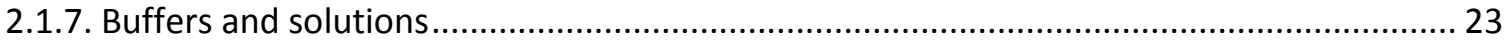

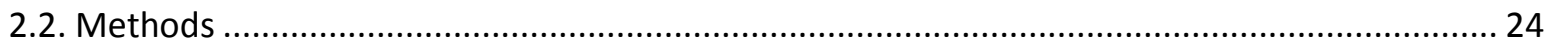

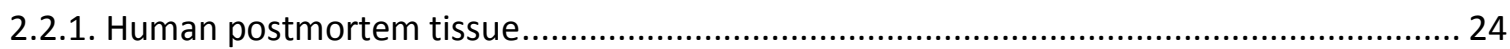

2.2.2. The 6-OHDA mouse model of PD and intracranial injection of AAV.shRNA ...................... 28

2.2.3. In vitro inhibition of ROCK2 in primary neuron cell culture .............................................. 40

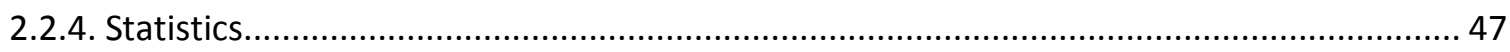

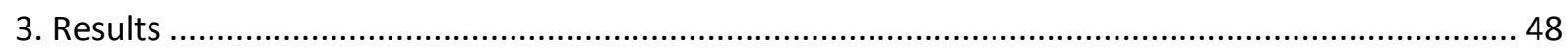

3.1. Analysing the human brain: Is there a regeneration failure in PD? ....................................... 48

3.1.1. Immunohistochemical characterization of patient cases ................................................. 48

3.1.2. Analysis of growth-associated proteins and presynaptic configuration ............................ 53

3.1.3. Investigation of the growth-inhibitory protein Rho kinase (ROCK2) in human brain sections

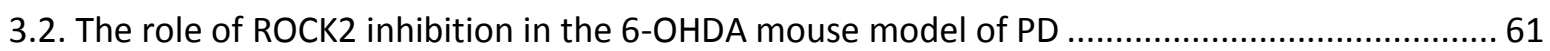


3.2.1. AAV mediated gene transfer leads to effective dsRed expression in neurons of the substantia nigra

3.2.2. shRNA-mediated ROCK2 downregulation increases dopaminergic neuron survival after 6OHDA intoxication

3.2.3. ROCK2 downregulation has only mild effects on dopaminergic fiber density and dopamine levels in the striatum. 65

3.2.4. ROCK2 downregulation via AAV-shRNA has only minor effects on behavioral outcome... 68

3.3. Effect of long-term ROCK modulation on synaptic vesicle dynamics in vitro ..........................72

3.3.1. Fasudil treatment has no negative effects on cell viability on DIV15 ........................... 72

3.3.2. Pharmacological ROCK inhibition alters synaptic vesicle dynamics in vitro ....................73

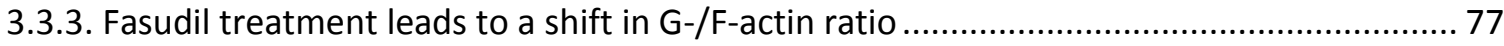

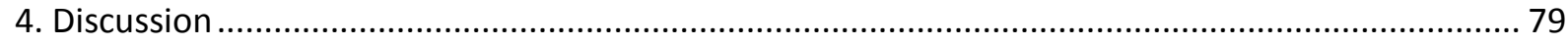

4.1. Is there intrinsic regenerative capacity or a regeneration failure in Parkinson's disease?....... 79

4.1.1. The investigated brain sections mirror characteristic features of PD ............................80 80

4.1.2. GAP-43 and synaptophysin expression are altered in the nigrostriatal system in PD ........82

4.1.3. There is diffuse ROCK2 expression in the nigrostriatal system of the human brain ..........84

4.1.4. ROCK2 expression is altered in striatal astrocytes and microglia ..................................85

4.2. The role of neuronal ROCK inhibition in the 6-OHDA mouse model of PD ............................ 88

4.2.1. shRNA mediated ROCK2 downregulation increases dopaminergic survival after 6-OHDA

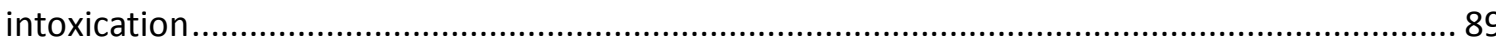

4.2.2. Neuronal ROCK2 downregulation has only minor effects on behavioral outcome ............91

4.3. The role of pharmacological ROCK inhibition on dynamic presynaptic function.................... 92

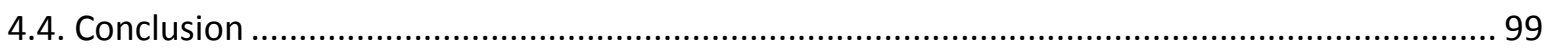

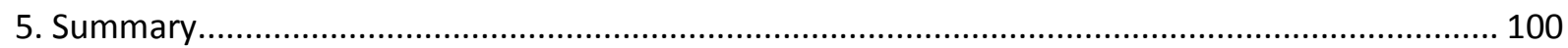

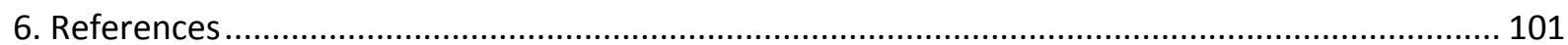

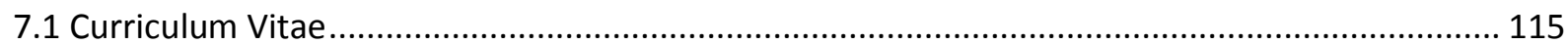

7.2. Publications .............................................................................................................. 116

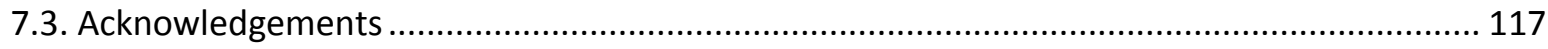




\section{Abbreviations}

6-OHDA: 6-hydroxydopamine

AAV: adeno-accociated virus

AD: Alzheimer's disease

ADP: adenosine diphosphate

AK: adenylate kinase

ALS: amyotrophic lateral sclerosis

amc: age-matched control

AP: anterior-posterior

APS: ammonium peroxide sulfate

ATP: adenosine triphosphate

a.u.: arbitrary units

BSA: bovine serum albumine

BDNF: brain derived neurotrophic factor

$\mathrm{CaCl}_{2}$ : calcium chloride

CERAD: Consortium to Establish a Registry for Alzheimer's disease

CCD: charge-coupled device

$\mathrm{CH}_{4} \mathrm{O}$ : methanol

$\mathrm{C}_{6} \mathrm{H}_{8} \mathrm{O}$ : citric acid

$\mathrm{C}_{2} \mathrm{H}_{4} \mathrm{O}_{2}$ : acetic acid

Cl: capsula interna

CNS: central nervous system

CNTF: ciliary neurotrophic factor

$\mathrm{CO}_{2}$ : carbon dioxide

CRMP2: collapsing response mediator protein 2

CSPG: chondroitin sulphate proteoglycan

Ctl: contralateral

Cy: cyanine

DA: dopamine

DAB: 3,3'-diaminobenzidine

DAPI: 4',6-diamidino-2-phenylindole 
DIV: days in vitro

DMEM: Dulbecco's Modified Eagle Medium

DOPAC: 3,4-dihydroxyphenylacetic acid

DPX: distrene-plastilizer-xylene

dsRed: Discosoma Red

DV: dorso-ventral

EAAT: excitatory amino acid transporter

ECL: enhanced chemiluminescence

EDTA: ethylenediaminetetraacetic acid

EGFP: enhanced green fluorescent protein

F-actin: filamentous actin

FCS: fetal calf serum

G-actin: globular actin

GAP-43: growth associated protein-43

GFAP: glial fibrillary acidic protein

GPCR: G-protein-coupled receptor

GPe: globus pallidus externus

GPi: globus pallidus internus

HBSS: Hank's balanced salt solution

$\mathrm{H}_{3} \mathrm{BO}_{3}$ : boric acid

$\mathrm{HCl}$ : hydrochloric acid

$\mathrm{HClO}_{4}$ : perchloric acid

$\mathrm{H}_{2} \mathrm{C}_{6} \mathrm{O}$ : ethanol

HD: Huntington's disease

HEPES: 2-[4-(2-hydroxyethyl) piperazin-1-yl] ethanesulfonic acid

$\mathrm{HNO}_{3}$ : nitric acid

$\mathrm{H}_{\mathbf{2}} \mathrm{O}$ : water

$\mathrm{H}_{2} \mathrm{O}_{2}$ : hydrogen peroxide

HPLC: high performance liquid chromatography

HRP: horseradish peroxidase

HS: horse serum

hsyn: human synapsin 
HVA: homovanillic acid

Iba1: ionized calcium binding adapter molecule 1

ICC: immunocytochemistry

IF: immunofluorescence

IHC: immunohistochemistry

Ipl: ipsilateral

IR: immunoreactivity/immunoreactive

$\mathrm{KCl}$ : potassium chloride

L-AA: L-ascorbic acid

LAS2: Iysis and stabilization2

LB: Lewy body

LF: left front

LH: left hind

LINGO-1: leucine rich repeat and Ig domain-containing Nogo receptor-interacting protein-1 LPA: phospholipid lysophosphatidic acid

MEM: minimum essential medium

$\mathrm{MgCl}_{2}$ : magnesium chloride

ML: medio-lateral

MLC: myosin light chain

MLCP: myosin light chain phosphatase

MPTP: 1-methyl-4-phenyl-1,2,3,6-tetrahydropyridine

$\mathrm{Na}_{2} \mathrm{~B}_{4} \mathrm{O}_{7}$ : sodium tetraborate

$\mathrm{NaCl}$ : sodium chloride

$\mathrm{NaHCO}_{3}$ : sodium hydrogen carbonate

$\mathrm{Na}_{2} \mathrm{HPO}_{4}$ : disodium hydrogen phosphate

$\mathrm{NaN}_{3}$ : sodium azide

$\mathrm{NaO}_{2} \mathrm{C}_{2} \mathrm{H}_{3}$ : sodium acetate

$\mathrm{NaOH}$ : sodium hydroxide

NC: caudate nucleus

NDD: neurodegenerative disorder

NDS: normal donkey serum 
NeuN: neuronal nuclei

NFT: neurofibrillary tangles

NgR1: Nogo receptor 1

NGS: normal goat serum

$\mathbf{N H}_{4} \mathrm{Cl}$ : ammonium chloride

NNP: no neuropathology

NR: red nucleus

NTF: neurotrophic factor

OMgp: oligodendrocyte-myelin glycoprotein

ON: over night

PBS: phosphate buffered saline

PC: cerebral peduncle

PD: Parkinson's disease

Pen/strep: penicillin/streptomycin

PFA: paraformaldehyde

$\mathbf{P I}(3,4,5) \mathbf{P}_{3}$ : phosphatidylinositol $(3,4,5)$ triphosphate

PLL: poly-L-lysine

PMI: post mortem interval

PPN: pedunculo pontine nucleus

PU: putamen

RBD: rho binding domain

RF: right front

RH: right hind

RNA: ribonucleic acid

ROCK: rho kinase

rpm: rounds per minute

RRP: readily releasable pool

RT: room temperature

SDS: sodium dodecyl sulfate

SDS-page: SDS-polyacrylamide gel electrophoresis

SEM: standard error of the mean

shRNA: small hairpin RNA 
siRNA: small interfering RNA

SN: substantia nigra

SNpc: substantia nigra pars compacta

SNpr: substantia nigra pars reticulata

S1P: sphingosine-1-phosphate

stim: stimulated/stimulation

STN: subthalamic nucleus

Synphy: synaptophysin

syt1: synaptotagmin1

TBS: tris-buffered saline

TBS-T: TBS-Tween20

TEMED: tetramethylethylenediamine

TH: tyrosine hydroxylase

TU: transforming unit

Tris: 2-amino-2-hydroxymethyl-propane-1,3-diol

TTX: tetrodotoxin

UMG: University Medicine Göttingen

VTA: ventral tegmental area

yc: young control 


\section{Introduction}

\subsection{Preface}

Improvements in the environmental and occupational conditions as well as advances in medical care resulted in an increased life expectancy in the industrialized world. However, higher age also comes with an increased risk for neurodegenerative diseases. These disorders are accompanied by physical or mental impairments and lead to a long period of suffering. Most of the pathogenic causes are still not known, thus no curative therapies are available.

\subsection{Neurodegenerative disorders}

The progressive damage or death of neurons in the central nervous system (CNS) is one of the main reasons for disability in industrialized countries. The underlying disorders strongly impair quality of life by deterioration of sensory, motor and higher cognitive functions. Neurodegeneration occurs mainly from spontaneous or inherited causes as well as from traumatic lesions and is mostly associated with atrophy of the central or peripheral nervous system, leading to reduced innervation of the target organs. The most frequently occurring neurodegenerative disorders (NDD) are Alzheimer's disease (AD), Parkinson's disease (PD), Huntington's disease (HD), amyotrophic lateral sclerosis (ALS) and prion diseases. Among the induction of neurodegeneration by inherited mutations of certain proteins, multifactorial endogenous pathomechanisms, for instance oxidative stress, excitotoxicity, protein misfolding and spontaneous mutations can result in protein accumulation and lead to the activation of signal-transduction pathways initiating cell death (Ross \& Poirier, 2004; Hutchins \& Barger, 1998; Martin, 2001). For most NDD, no curative therapy exists. Even though there are numerous therapies to relieve symptoms of these disorders, they cannot prevent the progression of pathology. In the last decades, research succeeded in discovering candidate molecules involved in the pathophysiology, but their particular roles are still not fully understood (Forman et al., 2004; Prusiner, 2001). Next to the necessity to develop strategies to improve symptomatic treatment and to elucidate the 
reasons for neurodegeneration, there is thus prime importance to establish therapies counteracting neuronal degeneration and facilitate regeneration.

\subsection{Parkinson's disease}

\subsubsection{Epidemiology and phenotype}

The first scientific description of the disorder was done by the British physician James Parkinson in 1817, when he examined six patients who showed the core clinical features comprising today's PD symptomatic. This includes resting tremor, flexed posture with a tendency to fall, bradykinesia, which is the paucity of voluntary movements and muscle rigidity. Moreover, additional symptoms are found in Parkinson's disease, for instance insomnia, dysphagia, urinary incontinence, pain and psychiatric disorders such as depression and dementia (Parkinson, 1817; Dexter \& Jenner, 2013; Dauer \& Przedborski, 2003; Smith et al., 2012). In industrialized countries the prevalence of PD is estimated with $0.3 \%$ of the entire population, but considering that it is an age-dependent disorder with an average disease onset at $\sim 60$ years, the prevalence increases to $1 \%$ in the population older than 60 years (Lau \& Breteler, 2006; Dexter \& Jenner, 2013) and is therefore the second most frequent NDD after AD. In 5\% of the affected patients the disease is inherited in the form of point missense mutations and multiplications of certain genes, while in the other $95 \%$ the disease occurs sporadically (Chartier-Harlin et al., 2004; Singleton et al., 2004; Federoff et al., 2003; Dauer \& Przedborski, 2003).

\subsubsection{Anatomical background and pathophysiology}

Parkinson's disease is a system disorder involving many parts of the brain. It's bestknown pathological hallmarks include the prominent loss of dopaminergic neurons in the substantia nigra (SN) in the midbrain, although the degeneration is not limited to this neuron type (Pillon et al., 1989; Pereira et al., 2012). These dark pigmented dopaminergic neurons, containing neuromelanin, are part of the basal ganglia and involved in complex regulatory circuits of voluntary movements. From the cell bodies located in the substantia nigra pars compacta $(\mathrm{SNpc})$ the unmyelinated axons project into the striatum, which is divided in the putaminal part and the caudate nucleus. Dopaminergic terminals provide input to the spiny 
striatal neurons and therefore modulate the activity of striatal cells mediated by two dopamine receptors D1 and D2 (Kandel et al., 2000; Purves et al., 2004). They provide excitatory input mediated by D1 type receptors on spiny cells targeting the internal globus pallidus (GPi) via the direct pathway, while D2 type receptors mediate inhibitory input on the spiny neurons and thereby inhibit activity of the external globus pallidus (GPe). Repressing input in the GPe leads to excitatory outputs of the subthalamic nucleus that in turn projects to the GPi and represents the indirect pathway. Afferent fibers of the GPi target the ventral nucleus of the thalamus that projects to the frontal cortex and back to the spiny neurons of the striatum (Parent \& Hazrati, 1995; Obeso et al., 2002; Crittenden \& Graybiel, 2011). The interconnection of cortical inputs into the striatum and substantia nigra pars reticulata (SNpr) mirrors the complexity and importance of these regulatory circuits to generate voluntary movements. Any imbalance in transmitter levels targeting the striatal spiny neurons leads to more or less pronounced motor dysfunctions. Therefore, degeneration of nigral dopaminergic neurons leads to dopamine depletion in the striatum and generates the characteristic symptoms of PD by underactivation of the GPi (Bernheimer et al., 1973; Hornykiewicz, 2001; Bergman \& Deuschl, 2002; Obeso et al., 2002; DeLong \& Wichmann, 2007) (see Figure 1.1.: Schematic diagram of the direct and indirect pathways of the basal ganglia in normal and PD conditions, from Smith et al., 2012). Due to the fact that the terminal loss of dopaminergic fibers is much more pronounced in the striatum than the dopaminergic cell death in the SNpc, it is assumed that the degeneration of the nigrostriatal system follows a dying back mechanism, starting at the axonal terminals and involves mechanisms of axonal degeneration (Burke \& O’Malley, 2013; Tönges et al., 2012). 

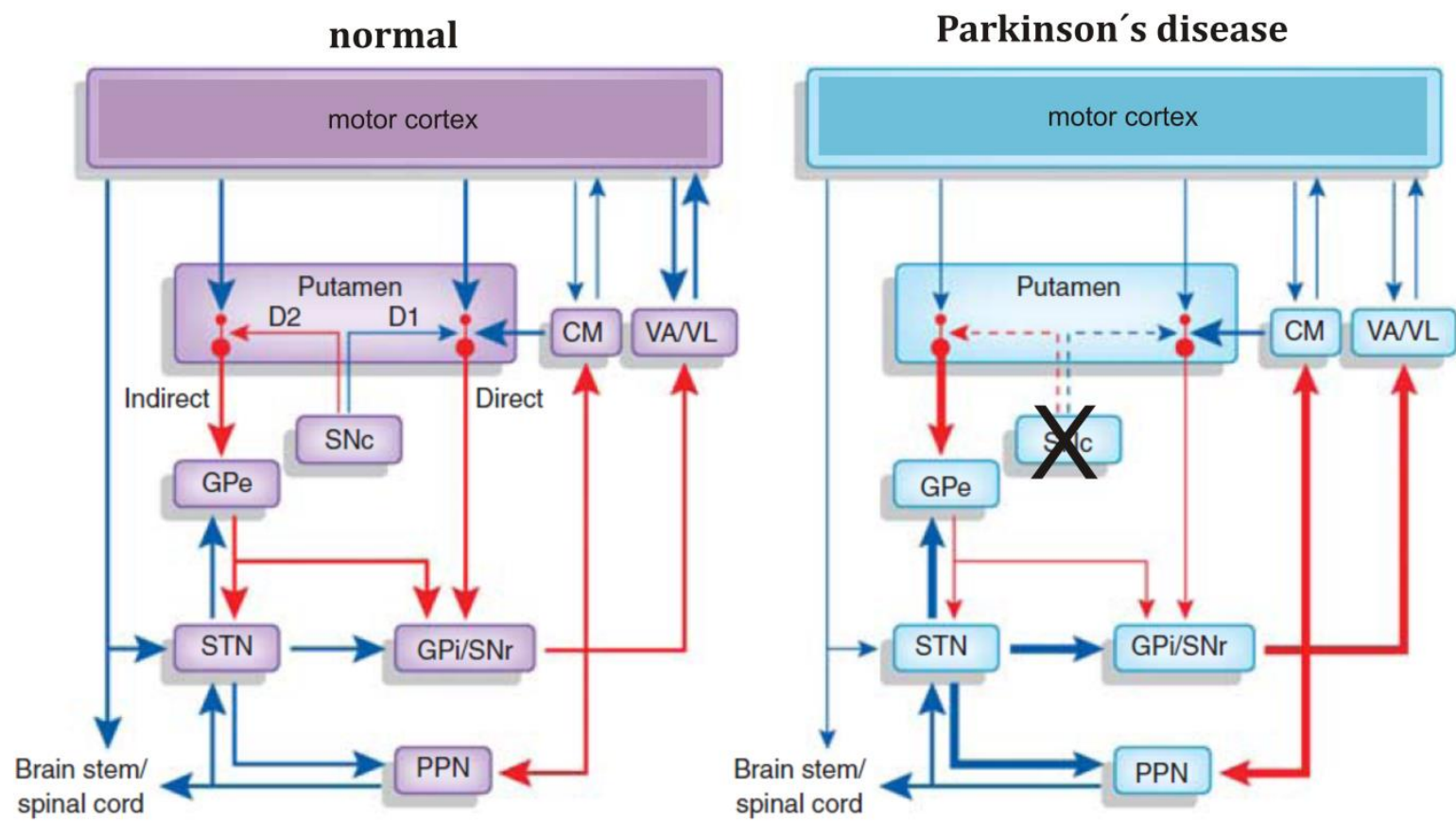

Figure 1.1: Scheme of the direct and indirect circuits of the basal ganglia in healthy and PD conditions. Red arrows denote inhibitory and blue arrows indicate excitatory input in the target area. The thickness of arrows signifies the strength of transmission. Eliminating output from the SNpc in Parkinsonism (right diagram), changes the transmission strength in the following structures resulting in less cortical input (modified from Smith et al., 2012).

\subsubsection{Etiology}

\subsubsection{Lewy bodies and their contents}

At the time of clinical disease onset, around $50 \%$ of the dopaminergic neurons are already degenerated, resulting in motor dysfunction (Fearnley \& Lees, 1991). One of the disorder's hallmarks discovered in 1912 by Friedrich Lewy, is the presence of intra cytoplasmatic inclusion bodies, later called Lewy bodies (LBs), which are found in the remaining dopaminergic neurons and their neurites in the SNpc, but also in other parts of the brain (Forman et al., 2004; Gibb \& Lees, 1988; Dickson et al., 2009). The role of the Lewy body, which also occur in other so-called Lewy body diseases, is still under debate. Following the theory of Braak, who proposes a staging of PD based upon an expanding LB load from the brainstem to cortical structures, they are have a detrimental function inducing neuronal death during disease progression (Braak et al., 2003; Braak \& Braak, 1991; Braak et al., 2003). On the other hand, in regard to their composition consisting mainly of neurofilaments, ubiquitin and alpha-synuclein and their wide distribution throughout the 
brain, LBs could also have protective properties by isolating toxic material from the surrounding cytoplasm and therefore promoting survival of the LB-containing neurons (Tompkins \& Hill, 1997; Burke \& Barnes, 2006; Parkkinen et al., 2011).

\subsubsection{Alpha-synuclein}

In 1997, Maria G. Spillantini discovered the main component of LBs, which is the 140 amino acid protein alpha-synuclein (Spillantini et al. 1997; Spillantini et al. 1998). Normally, this soluble protein is abundantly localized in the cytosol and presynaptic nerve terminals. The physiological role of alpha-synuclein is associated with the SNAREing of synaptic vesicles, reversibly linking them to membranes by containing an $\mathrm{N}$-terminus similar to apolipoproteins (Sidhu et al., 2004; Lashuel et al., 2013; Chandra et al., 2003). Additional functions are very likely and not yet completely understood.

Alpha-synuclein protein mutation or misfolding leads to aggregation of cytosolic oligomer and protofibrillar alpha-synuclein, which is widely accepted to be the toxic species of this protein. Thus, it is likely that fibrils, which are encased in the LBs, are less toxic (Choi et al., 2013; Rochet et al., 2004; Bucciantini et al., 2002). In the alpha-synuclein encoding gene (SNCA) there are five alpha-synuclein missense mutations causing PD known today: A53T, A30P, E46K and the recently discovered G51D and H50Q (Kiely et al., 2013; Proukakis et al., 2013). Mutations and polymorphisms in the SNCA gene can lead to the inherited disease by favouring the formation and accumulation of oligomeric and fibrillar structures (Lashuel et al., 2013; Chartier-Harlin et al., 2004; Lázaro et al., 2014). In addition to mutations or multiplications in the SNCA gene, other mutated proteins were discovered to cause the rare forms of familiar PD, e.g. Parkin, Pink1, LRRK2, DJ-1 and ATP13A2 (Burke \& O’Malley, 2013; Dawson \& Dawson, 2003; Dexter \& Jenner, 2013).

\subsubsection{Oxidative stress}

Dysfunction of proteins can also be triggered by oxidative stress generated by reactive oxygen species (ROS), catalyzed by transition metals or the dysfunctional production of ATP from the mitochondrial respiratory chain complex I. Mitochondria normally release powerful oxidants as by-products of energy production, but it is still not clear whether the 
impairment of complex I is the cause or the consequence of neurodegeneration (Yan et al., 2013; Dauer \& Przedborski, 2003; Blesa et al., 2012; Dexter \& Jenner, 2013).

The specific vulnerability of dopaminergic neurons in PD could result from different additional factors: The neurotransmitter dopamine is degraded by enzymatic deamination through monoamine oxidases (MAOs) producing 3,4-dihydroxyphenylacetic acid (DOPAC) and $\mathrm{H}_{2} \mathrm{O}_{2}$. The interaction of transition metals and $\mathrm{H}_{2} \mathrm{O}_{2}$ results in the production of toxic hydroxyl radicals while dopamine can be oxidized and form quinines, superoxide free radicals and hydrogen peroxide (Sidhu et al., 2004). Excess dopamine amounts and the consequent generation of toxic quinones and semiquinones are prevented by the removal of excessed catecholamines from the cytosol in the synthesis of neuromelanin, which occurs as a characteristic substance in dopaminergic neurons of the SN (Graham, 1978). Additionally to remove excess catecholamines from the cytosol, it chelates redox-active metals by forming complexes and therefore reducing the production of hydroxyradicals (Zecca et al., 2003). Therefore, the combination of mitochondria-related energy failure, dysfunctional neuromelanin synthesis leading to ROS production from dopamine metabolism and altered scavenging capacity of transition metal levels may contribute equally to neurodegeneration of especially nigral dopaminergic neurons.

\subsubsection{Glial reactions in PD}

Another hallmark in neurodegenerative disorders is the accompanying glial infiltration to the damaged region. In the healthy brain microglia are found throughout the parenchyma and are constantly active. Their ramified processes scan the extracellular space for cues revealing injury. After sensing, microglia move to the place of damage, where they agglomerate and become phagocytotic to remove cell debris from injury (McGeer \& McGeer, 2008; Hanisch \& Kettenmann, 2007). This inflammatory reaction occurs also in the SN of PD patients and it was shown that microglia infiltration evokes dopaminergic cell damage in vitro and in vivo. After activation, microglia are thought to produce large amounts of superoxide radicals, which in turn could be responsible for dopaminergic damage via oxidative stress (Hirsch \& Hunot, 2009; Hunter et al., 2007; McGeer \& McGeer, 2008; Su et al., 2009; Kim \& Joh, 2006). 
The role of astrocytes in the lesioned CNS is also not completely elucidated yet. Some of the normal functions of astrocytes in the brain are the maintenance of neurons by supply with essential nutrients and neurotrophic factors, the regulation of ion concentrations and the uptake and metabolism of extracellular neurotransmitters (Maragakis \& Rothstein, 2006). After injury, activated astrocytes are building a wall-like structure in damaged regions, the so-called astrogliosis. In PD, astroglia seems to play a neuroprotective role against oxidative stress, depending on the quantity of the antioxidatively acting enzyme glutathione peroxidase, which was inversely correlated with the severity of dopaminergic degeneration in human post mortem brains (Damier et al., 1993).

\subsection{Current treatment of PD and future approaches}

\subsubsection{Symptomatic treatment}

Until now there is no curative treatment for PD available and the medication used in PD is merely symptomatic. Available treatments to attenuate symptoms of PD are usually orally applied, for example dopamine agonists, levodopa (as the precursor of dopamine biosynthesis), monoamine oxidase $\mathrm{B}$ (MAOB) inhibitors (to prevent dopamine degradation after release) and anti-glutamatergic or anti-cholinergic substances to adjust transmitter levels in the basal ganglia. Alternatively, some substances can be delivered continuously via subcutaneous or intrajejunal infusions (Dunnett \& Björklund, 1999; Obeso et al., 2010). Deep brain stimulation is reserved for more advanced cases of the disease (Deuschl \& Bergman, 2002; Stefani et al., 2007).

\subsubsection{Future approaches}

One of the difficulties in PD therapy arises from the very late onset of motor symptoms, when already more than the half of the nigrostriatal projections is degenerated (Bernheimer et al., 1973). Because until now there is no biomarker approved that can predict onset of motor symptoms, the main challenge would be to either prevent the remaining neurons from further degeneration or to facilitate restorative neuronal growth after diagnosis of PD. 


\subsubsection{Regenerative strategies}

There are several approaches to prevent neurons from dying as well as to enhance neuronal regeneration in vivo and in vitro. It is now also established that also adult aged neurons have the intrinsic ability to sprout, although it is lower than in young or even embryonic neurons (Fawcett, 1992; Verma et al., 2005). In animal models of PD reinnervation of the striatum by nigral dopaminergic fibers was observed after toxin-induced nigrostriatal degeneration (Hagg \& Oudega, 2006; Finkelstein et al., 2000; Mitsumoto et al., 1998). One indicator of regenerative growth or sprouting of neurons in the CNS is the expression of growth associated proteins (GAP), such as GAP-43, which is known to regulate membrane motility and neuronal plasticity by modulating F-actin polymerisation (Schmidt, 2004; Laux et al., 2000). Thus, the lesioned dopaminergic system generally has the ability to regenerate, but it is unclear whether compensatory regeneration takes place in Parkinson's disease brains or if this mechanism is impaired in pathology.

\subsubsection{Neurotrophic factors}

The application of neurotrophic factors (NTF) in several pre-clinical studies revealed a promising potential to facilitate nigrostriatal restoration, but these approaches mostly failed in human trials (Aron \& Klein, 2011; Hidalgo-Figueroa et al., 2012; Winkler et al., 1996). Nevertheless, there were single promising results in cases of unilateral intrastriatal infusion of the glial cell-line derived neurotrophic factor (GDNF), which is essential for development and survival of dopaminergic neurons in vitro and in vivo (Tomac et al., 1995; Kirik et al., 2004; Clarkson et al., 1997). For example, the putaminal infusion in a 62-year old PD patient with advanced disease resulted in improved motor performance within 24 months after continuous infusion. Post mortem immunohistology demonstrated a local increase in tyrosine hydroxylase (the rate limiting enzyme for dopamine synthesis and marker for dopaminergic neurons), accompanied by enhanced immunoreactivity for growth-associated protein-43, which speaks in favour of fiber sprouting to the treated side (Love, 2005). 


\subsubsection{Overcoming growth restricting obstacles}

Extracellular cues from surrounding cells, e.g. astroglia or oligodendrocytes, are involved in growth inhibition and provoke regenerative failure (Kottis et al., 2002; Cafferty et al., 2010; Yiu \& Zhigang, 2009). Via neuronal surface receptors growth inhibitory signaling cascades are activated involving Rho associated kinase (ROCK), which results in the stabilization of the dynamic actin cytoskeleton and growth cone collapse (Gehler et al., 2004; Gallo \& Letourneau, 2004; Mueller et al., 2005). Recently our group could show that ROCK inhibition improves regenerative neurite outgrowth, axonal regeneration and neuronal survival in different in vitro and in vivo models of optic nerve trauma, Parkinson's disease and amyotrophic lateral sclerosis (Lingor et al., 2007; Bermel et al., 2009; Tatenhorst et al., 2014; Tönges et al., 2012; Günther et al., 2014; Tönges et al., 2014). Pharmacologically induced ROCK inhibition via the small molecule inhibitors fasudil or Y-27632 stimulated cytoskeleton reorganization, neurite outgrowth and axonal regeneration, while the improved survival was mediated by the activation of survival cascades, e.g. Akt/PKB, which were also activated by trophic factors, such as the ciliary neurotrophic factor (CNTF) (Chadi et al., 1993; Peterson et al., 2000) in vitro and in vivo (Lingor et al., 2007; Lingor et al., 2008; Bermel et al., 2009).

In the 1-methyl-4-phenyl-1,2,3,6-tetrahydropyridine (MPTP) and the 6-hydroxydopamine (6-OHDA) toxin-induced animal models of PD, the respective toxin is applied systemically (MPTP) or via intracranial injection (6-OHDA). MPTP crosses the blood brain barrier and the MAO-B from astrocytes subsequently metabolizes it into its active ion $\mathrm{MPP}^{+}$. 6-OHDA, on the other hand, has to be applied directly to the brain area of interest. Both active toxins are taken up into dopaminergic cells via the dopamine transporter (DAT), where they inhibit the complex I of the mitochondria, which leads to production of ROS, oxidative stress, energy failure and consequently to the degeneration of the dopaminergic cell (Cannon \& Greenamyre, 2010; Blesa et al., 2012; Dauer \& Przedborski, 2003).

As we could previously demonstrate, the pharmacological ROCK inhibitor fasudil induced enhanced dopaminergic cell survival in the SN of MPTP intoxicated mice, accompanied by a preservation of dopaminergic terminals in the striatum and improved motor performance of the animals (Tönges et al., 2012). Additionally, in the 6-OHDA model, ROCK inhibition via fasudil resulted in increased striatal DOPAC levels at 12 weeks after intoxication, indicating regenerative sprouting (Tatenhorst et al., 2014). 
ROCK inhibition via fasudil or Y-27632 not only results in beneficial effects on neurons, but also on astrocytes and microglia (Lau et al. 2012; Barcia et al. 2012; Yu et al. 2010; Monnier et al. 2003). Here, pharmacological inhibition of ROCK induces a morphological change in astrocytes, accompanied by determined changes in the astrocytic transcriptome, as for example the increase in the expression levels of the brain derived neurotrophic factor (BDNF) and the excitatory amino acid transporters (EAATs), indicating a astrocytic phenotype which supports neuronal survival (Lau et al. 2012; Lau et al. 2011). Furthermore, pharmacological ROCK inhibition resulted in reduced numbers of microglia engulfing dopaminergic neurons and attenuated cell loss in the MPTP animal model of PD, suggesting a preservation of degenerating neurons from phagocytotic elimination by activated microglia in regions of nigrostriatal cell death (Barcia et al. 2012; Villar-Cheda et al. 2012).

\subsubsection{The ROCK signaling cascade}

ROCK is a serine/threonine (Ser/Thr) protein kinase with a molecular mass of 160 kDa. Two different genes encode two ROCK isoforms: ROCK1 (or ROCK $\beta$ ) is mainly expressed in non-neuronal tissue, but it is also found in synaptic structures of motoneurons in the rat brain, while ROCK2 (or ROCK $\alpha$ ) is the dominant isoform in neurons of the vertebrate brain (Gonzalez-Forero et al., 2012; Komagome et al., 2000; Hashimoto et al., 1999) although its abundance in the human brain is not sufficiently investigated yet.

The catalytic domain of ROCK is located at the amino terminus, while the carboxyl terminus contains a pleckstrin-homology region with a cysteine rich-repeat sequence. The central part encompasses a coiled-coil-forming domain, containing the domain binding site for the GTP-bound Rho (RBD), which is the activator of ROCK. In the absence of GTP-Rho ROCK is forming an auto-inhibitory loop by the binding of the C-terminus to the kinase domain. In this conformation ROCK is inactive (Ishizaki et al., 1996; Shi \& Wei, 2008; Mueller et al., 2005).

The extraneuronal cellular composition in the CNS is a major limitation for regeneration mainly mediated by extracellular signaling from myelin or lesion-induced scar tissue via the ROCK cascade (Yiu \& Zhigang, 2009; Mueller et al. 2005). These extracellular cues consist of myelin-associated proteins, such as Nogo-A, myelin-associated glycoprotein 
(MAG), oligodendrocyte-myelin glycoprotein (OMgp) and several proteoglycans as well as by ephrins, semaphorins and chondroitin sulphate proeteoglycans (CSPGs), which are either normally expressed in CNS or upregulated after brain injury, for example in oligodendrocytes and astrocytes (Cafferty et al., 2010; Kottis et al., 2002; Yiu \& Zhigang, 2009; Goldshmit et al., 2006; Goldberg et al., 2004; Monnier et al., 2003).The binding of the myelin-associated ligands to a neuronal receptor complex, comprising the Nogo receptor 1 (NgR1), the leucine rich repeat and Ig domain-containing Nogo receptor-interacting protein-1 (LINGO-1) and the p75 neutrophin receptor $\left(p 75^{\mathrm{NTR}}\right.$ ) or TROY, on the neuronal surface results in the transformation of the inactive GDP-bound small GTPase RhoA into the active GTPase RhoA (Mi et al., 2004; Dudek \& Garcia, 2003). The phospholipid lysophosphatidic acid (LPA) as well as sphingosine-1-phosphate (S1P), which can be secreted from various cells and are present in several biological fluids (Eichholtz et al., 1993; Fyrst \& Saba, 2011), can stimulate the neuronal G-protein-coupled receptor (GPCR), which leads to the activation of GTPase RhoA (Goetzl, 2007; Seasholtz et al., 1999). Activated GTPase RhoA is binding at the RhoA binding domain of ROCK, causing thereby a conformational change of the kinase by abandoning its auto-inhibitory conformation and turning it into the active state (Amano et al., 2010; Ishizaki et al., 1996; Mueller et al., 2005). ROCK activation leads to an increased kinase activity, a translocation to cellular membranes and to phosphorylation of diverse target proteins. One of the main downstream targets is the myosin light chain (MLC), which is directly phosphorylated by ROCK and thereby stimulating interaction of actin-myosin contractility and the formation of stress fibers (Totsukawa et al., 2000; Raad et al., 2012). Additionally, ROCK activity leads to phosphorylation of the MYPT1 subunit of the myosin light chain phosphatase (MLCP), that results in less MLCP activity and therefore indirectly in increased amounts of phosphorylated MLC (Amano et al., 2010; Shi \& Wei, 2008). Another function of ROCK is to phosphorylate LIM-kinase and therefore increase its activity leading to more kinase activity towards the small actin-depolymerization factor cofilin, which in turn is inactivated by phosphorylation and stopped in its activity, what results in stress fiber formation and growth cone collapse (Maekawa, 1999). Active cofilin is maintaining a rapid filament assembly and disassembly of filamentous actin (F-actin) at the so called pointed ends by generating G-actin monomers through depolymerization at low concentrations of monomeric actin (Meyer \& Feldman, 2002). 
Further downstream targets of ROCK are the collapsing response mediator protein 2 (CRMP2), which plays an essential role in semaphorin-mediated axonal guidance during CNS development (Mueller et al., 2005) and proteins associated with the interconnection of the F-actin cytoskeleton and the plasma membrane, such as adducin or ezrin, radixin and moesin (ERMs) (Rêdowicz, 2002). ERMs are required for the formation of focal adhesion complexes and stress fibers, regulating cell-cell-junction dynamics, membrane ruffling and cell motility (Arpin et al., 2014). In regard to its function in synapses, ROCK is also thought to modulate dynamic neuronal physiology, neurotransmitter release and electrical activity (GonzalezForero et al., 2012). The actin cytoskeleton builds a stable, intricate network of several synaptic protein-protein interactions that is closely linked to synaptic vesicles and active zones and thereby modulating the guidance of synaptic vesicles to the plasma membrane (Dillon \& Goda, 2005; Brodin et al., 2000; Morales et al., 2000; Sakaba \& Neher, 2003). There is evidence that ROCK activity is necessary to maintain transmitter release and the motor output on presynapses from hypoglossal motoneurons in vitro (Gonzalez-Forero et al., 2012), but the exact underlying mechanisms are not fully understood.

Apart from the regulation of the cytoskeleton, ROCK signaling is involved in protein synthesis and cell survival. The protein tyrosine phosphatase and tensin homolog (PTEN) mediated survival cascades are well-known from cancer studies, where PTEN activation leads to tumor suppression (Guertin \& Sabatini, 2007; McCarroll et al., 2014). However, PTEN can be also directly phosphorylated by ROCK (Li et al., 2005), which leads to a decrease in intracellular levels of phosphatidylinositol $(3,4,5)$ triphosphate $\left(\operatorname{PI}(3,4,5) \mathrm{P}_{3}\right)$ by dephosphorylation leading to decreased activity of the serine-threonine kinase Akt, which in turn regulates the activity of Ras homolog enriched in brain (Rheb) (Meier et al., 2009; Dudek et al., 1997; Stambolic et al., 1998). Briefly, ROCK indirectly decreases the Rheb activity resulting in the inhibition of the mammalian target of rapamycin (mTOR) subunit mTORC1, thereby suppressing signals for cell growth and survival by negatively controlling mRNA translation, ribosome biogenesis, autophagy and metabolism (Guertin \& Sabatini, 2007; Park et al., 2011). Furthermore ROCK inhibition decreases cleaved caspase3 activity and therefore leads to suppression of caspase 3 mediated apoptotic signaling (Koch et al., 2014). The upstream activators and downstream targets of ROCK are summarized in Figure 1.2. 


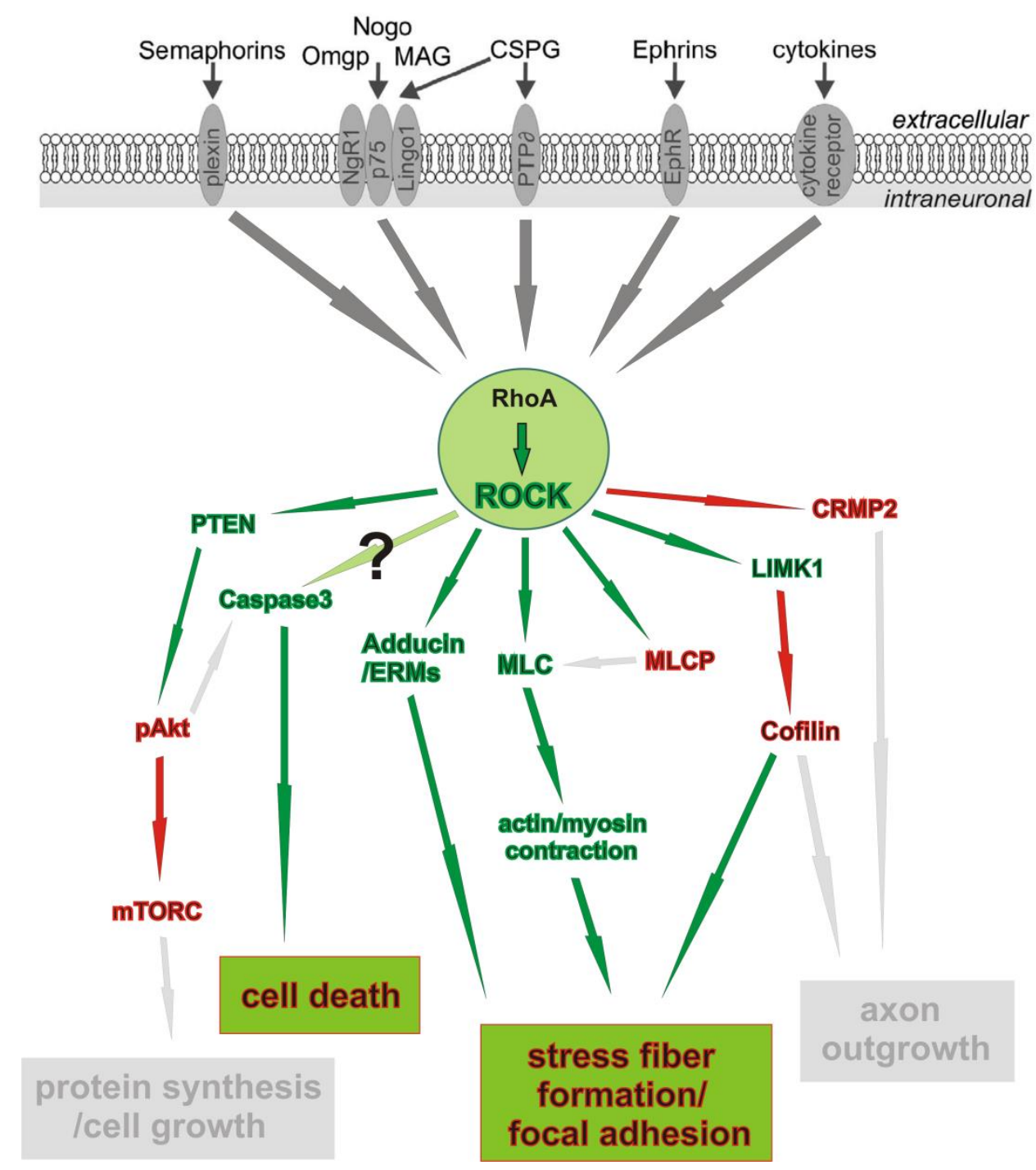

Figure 1.2: Scheme summarizing upstream activators and downstream targets of ROCK. Binding extracellular ligands propagate growth inhibitory cues through RhoA activation to activate ROCK (green). Phosphorylation of downstream targets (green) leads to actin cytoskeletal reorganisation, which results in stress fiber formation and growth cone collapse. Next to PTEN phosphorylation, ROCK activity furthermore results in negative regulation of downstream proteins (red), leading via mTORC to suppression (light grey) of cell growth and survival. These pathways might all be inverted after ROCK inhibition leading to axon outgrowth, cell growth and survival (modified from Koch et al., 2014).

\subsubsection{ROCK inhibition via fasudil}

The important role of the ROCK signalling cascade for survival and growth of neuronal cells became clear by inhibiting its activity with different substances. One of these ROCK inhibitors is the isoquinoline derivative fasudil, which in Japan is in clinical use since 1995 for 
the treatment of cerebral vasospasms following subarachnoid haemorrhage (Mueller et al., 2005). After fasudil administration over a time period of 14 days, patients suffering from severe subarachnoid haemorrhage showed reduced vasospasm and there were decreased numbers of patients with vasospasm-related clinical deterioration (Shibuya et al., 1992). Additionally, fasudil treatment was also employed in patients suffering from a stable angina pectoris, resulting in prolonged exercise time in the treadmill exercise testing without blood pressure and heart rate change (Shimokawa et al., 2002). Fasudil treatment was well tolerated in both studies and did not cause any serious side effects. In treatment of vasospasm fasudil operates via ROCK inhibition and the activation of MLCP, thereby antagonistically modulating the calcium sensitization for the $\mathrm{Ca}^{2+}$-depending muscle contraction and preventing vascular smooth muscle from inappropriate coronary hypercontraction, presumably caused by enhanced ROCK expression, which was also observed in a porcine model for vasospasms (Shimokawa et al., 2002).

As mentioned above, ROCK inhibition via fasudil leads in pre-clinical studies to attenuated neuronal cell loss and to enhanced neurite regenerative responses in different cell culture trials and in vivo models for neurode- and regeneration (Lingor et al., 2007; Lingor et al., 2008; Bermel et al., 2009; Tatenhorst et al., 2014; Tönges et al., 2012; Günther et al., 2014; Tönges et al., 2014, see 1.3.: Treatments of PD and promising candidates). However, fasudil is not a completely specific ROCK inhibitor. There is no special selectivity for ROCK1 and ROCK2 (Tang et al., 2013) and it was shown that fasudil affects several other kinases, for example AMP-activated protein kinase (AMPK) or phosphorylase kinase (PHK) (Davies et al., 2000; Cohen, 2002). Therefore it is important to first delineate the proregenerative effects and the neuroprotective mechanisms observed in vitro and in vivo more particularly to the specific inhibition of the Rho kinase in order to understand more about the mode of action of fasudil. Because it is used in Japan as licensed medication in humans, the safety profile and the pharmacokinetic behaviour is well-known, which makes fasudil a promising candidate for further evaluation in the treatment of neurodegenerative diseases.

\subsubsection{Viral vector gene transfer}

Viral vectors are employed to deliver particular DNA sequences into cells in vitro and in vivo due to their ability to infect cells and express their genome. The usage of adeno- 
associated viral (AAV) vectors seems to be a safe tool, since the wild-type virus is not causing disease in mammals per se (During \& Leone, 1995; Flotte \& Carter, 1995). The serotype of a virus confers different transduction properties dependent on the affinity and presence of specific receptors for AAV capsids (Tenenbaum, 2004). For example, it has been shown that $<5 \%$ of all transduced cells after stereotactical injection of the serotype AAV2 into the mouse striatum or substantia nigra are glia cells, while virtually all transfected cells were neurons (Tenenbaum et al., 2003; Bartlett et al., 1998; McCown et al., 1996; Klein et al., 1999).

Next to the introduction of foreign DNA sequences into the target cells, there is also the possibility to interfere with the cell genome by viral vectors resulting in the expression of small hairpin RNAs (shRNA) (Reynolds et al., 2004). Once introduced into the cell, the shRNAs are recognised by a RNase III family nuclease called Dicer and processed into functional siRNA, which enables complete pairing with the messenger RNA (mRNA) of the target gene followed by subsequent degradation of the target transcript (Cheng et al., 2003; Paddison et al., 2002). The introduction of viral vector-mediated RNA interference offers the possibility to target selected genes from particular cells downregulating their expression without regulating the transcription of other genes.

\subsection{Aims and goals}

Because Parkinson's disease can be diagnosed only when the characteristic motor symptoms occur following the degeneration of more than half of the nigrostriatal projections, it is of great interest to find ways to impede further cell loss and to facilitate neuronal regenerative responses to stop disease progression and to restore impaired motor functions.

In order to evaluate whether there is a specific intrinsic regeneration failure in dopaminergic neurons, in this work, human brain sections were examined by immunohistochemistry. To this, the expression levels and distribution of growth-associated proteins and the growth-inhibitory ROCK protein were evaluated in the brains of patients, who suffered from Parkinson's disease as well as from young and age-matched controls post mortem.

In the second part of the thesis, I focused on the role of ROCK2 in an animal model for PD. From recent studies we already knew that pharmacological inhibition of ROCK results 
in prolonged survival and improved regeneration in models of neurodegeneration in vitro and in vivo. Now we wanted to determine, if these effects were specifically mediated by the regulation of the ROCK2 isoform. Since there was no specific pharmacological inhibitor for ROCK2 available, we used viral vector-mediated introduction of a ROCK2 small-hairpin RNA (ROCK2-shRNA) into the substantia nigra of 6-OHDA-intoxicated mice to study the neuronspecific effect of ROCK2-downregulation on dopaminergic survival and degeneration. The unilateral striatal injection of the toxin and the ipsilateral introduction of the ROCK2-shRNA therefore enabled the investigation of specific neuronal effects of ROCK2-gene silencing on dopaminergic neuron with prime relevance to PD.

In the last part, I wanted to elucidate the impact of long-term ROCK inhibition especially on synaptic vesicle dynamics. Investigating the mode of action of fasudil on the synaptic actin cytoskeleton in primary hippocampal neuron cultures would give us further insights into the mechanistic action of ROCK inhibition. This is interesting, because the effects of ROCK inhibition on neurotransmitter release and signal transmission in neuronal circuits could additionally play an important role in the treatment of PD and other neurodegenerative diseases. 


\section{Materials and Methods}

\subsection{Materials}

\subsubsection{List of companies}

Abcam (Cambridge, UK); Agfa (Köln, Germany); Applichem (Darmstadt, Germany); Beckman Coulter Inc. (Krefeld, Germany); Biochrom (Berlin, Germany); BIO-RAD (München, Germany); Biozol (Eching, Germany); Braun (Melsungen, Germany); Calbiochem, by Merck Millipore (Darmstadt, Germany); Canon (Krefeld, Germany); Carl Zeiss Microimaging (Göttingen, Germany); Cell Signalling Technology (Cambridge, UK); Charles River (Sulzfeld, Germany); Corel Corporation (Ottawa, Canada); Cytoskeleton Inc. (Denver, USA); DAKO (Hamburg, Germany); David Kopf Instruments (Tujunga, USA); Dianova (Hamburg, Germany); Dionex, by Thermo Fisher Scientific GmbH (Dreieich, Germany); Dremel (Leinfelden-Echterdingen, Germany); Ecuphar (Greifswald, Germany); Eppendorf (WesselingBerzdorf, Germany); ESA (Bedfort, USA); Fluka (Steinheim, Germany); GE Healthcare (Chalfont St. Gilles, UK); Gibco (Karlsruhe, Germany); GMI (Minnesota, USA) GraphPad Software Inc. (La Jolla, USA); Hitachi GmbH (Düsseldorf, Germany); IVC, Tecniplast (Hohenpeißenberg, Germany); Jackson ImmunoResearch Laboratory Inc. (Suffolk, UK); KyensLab Incorporated (Tokyo, Japan); Leica Microsystems (Mannheim, Germany); Lonza (Köln, Germany); Lc Labs (Woburn, USA); Micro Bright Field Inc. (MBF, Colchester, VT, USA); Medistar (Ascheberg, Germany); Menzel (Braunschweig, Germany); Merck (Darmstadt, Germany); Millipore (Darmstadt, Germany); N.I.H. (Bethesda, USA); Nikon Instruments (Düsseldorf, Germany); Noldus (Wageningen, the Netherlands); Okolab (Pozzuoli, Italy); PAA (Pasching, Germany); Precellys by Peqlab (Erlangen, Germany); Roth (Karlsruhe, Germany); Santa Cruz (Heidelberg, Germany); Sarstedt (Nümbrecht, Germany); Sigma Aldrich (Taufkirchen, Germany); Serotec (Oxfort, UK); Serumwerk (Bernburg, Germany); Synaptic Systems (Göttingen, Germany); Systat Software GmbH (Erkrath, Germany); Teclapharm (Lüneburg,Germany); The MathWorks Inc. (Natick, USA); Thermo Scientifc (Waltham, USA); UgoBasile (Comerio, Italy); Vector Laboratories (Burlingame, USA); Wako Pure Chemical Industries (Neuss, Germany); World Precision Intruments (WPI, Berlin, Germany); Worthington (Lakewood, NJ, USA); Zytomed (Berlin, Germany) 
2.1.2. Chemicals

\begin{tabular}{|c|c|}
\hline 6-OHDA (Sigma) & glutamine (Lonza) \\
\hline 5-Fluoro-2'-desoxyuridine (Sigma) & glycerol (Roth) \\
\hline $30 \% \mathrm{H}_{2} \mathrm{O}_{2}$ (Applichem) & glycine (Applichem) \\
\hline $0,9 \%$ saline (Braun) & $\mathrm{H}_{3} \mathrm{BO}_{3}$ (Sigma) \\
\hline apomorphine (Teclapharm) & HBSS (Gibco) \\
\hline APS (Sigma) & $\mathrm{HClO}_{4}($ Merck) \\
\hline acrylamide (Applichem) & $\mathrm{HCl}$ (Roth) \\
\hline B-27 Supplement (Gibco) & HEPES (Applichem, Merck) \\
\hline Bepanthene (Braun) & Histoacrylic glue (Braun) \\
\hline BSA (Applichem) & $\mathrm{HNO}_{3}(\mathrm{Sigma})$ \\
\hline $\begin{array}{l}\text { BSA, immunoglobulin and protease free } \\
\text { (Jackson ImmunoResearch Laboratory Inc) }\end{array}$ & HPLC water (Merck) \\
\hline $\mathrm{CaCl}_{2}$ (Merck) & HS (Biochrom) \\
\hline $\mathrm{C}_{2} \mathrm{H}_{4} \mathrm{O}_{2}$ (Roth) & Isopropanole (Applichem) \\
\hline $\mathrm{C}_{6} \mathrm{H}_{8} \mathrm{O}_{7} * \mathrm{H}_{2} \mathrm{O}$ (Roth) & $\mathrm{KCl}$ (Merck) \\
\hline $\begin{array}{l}\text { DAB peroxidase substrate Kit, SK-4100 } \\
\text { (Vector laboratories) }\end{array}$ & ketamine (Medistar) \\
\hline DAPI (Sigma) & L-AA (Sigma) \\
\hline DMEM (Gibco, PAA) & L-Cysteine (Fluka) \\
\hline DPX (Fluka) & luminol (Calbiochem) \\
\hline Dual Proteine Standard (BIO-RAD) & MEM (Gibco) \\
\hline EDTA (Applichem, Merck) & Metapyrin (Serumwerk) \\
\hline ethanol absolute (Applichem, Merck) & methanol (Applichem, Roth) \\
\hline ethanol denatured (Applichem, Merck) & mineral oil (Sigma) \\
\hline fasudil (Lc Labs) & $\mathrm{MgCl}_{2}$ (Merck) \\
\hline FCS (Biochrom, PAA) & mowiol-488 (Merck, Sigma) \\
\hline $\begin{array}{l}\text { G-actin/F-actin in Vivo Assay Kit } \\
\text { (Cytoskeleton Inc.) }\end{array}$ & $\mathrm{Na}_{2} \mathrm{~B}_{4} \mathrm{O}_{7}($ Sigma $)$ \\
\hline glucose (Merck) & $\mathrm{NaCl}$ (Merck) \\
\hline GlutaMAX Supplement (Gibco) & $\mathrm{NaHCO}_{3}($ Merck) \\
\hline $\mathrm{Na}_{2} \mathrm{HPO}_{4}$ (Merck) & uridine (Sigma) \\
\hline
\end{tabular}




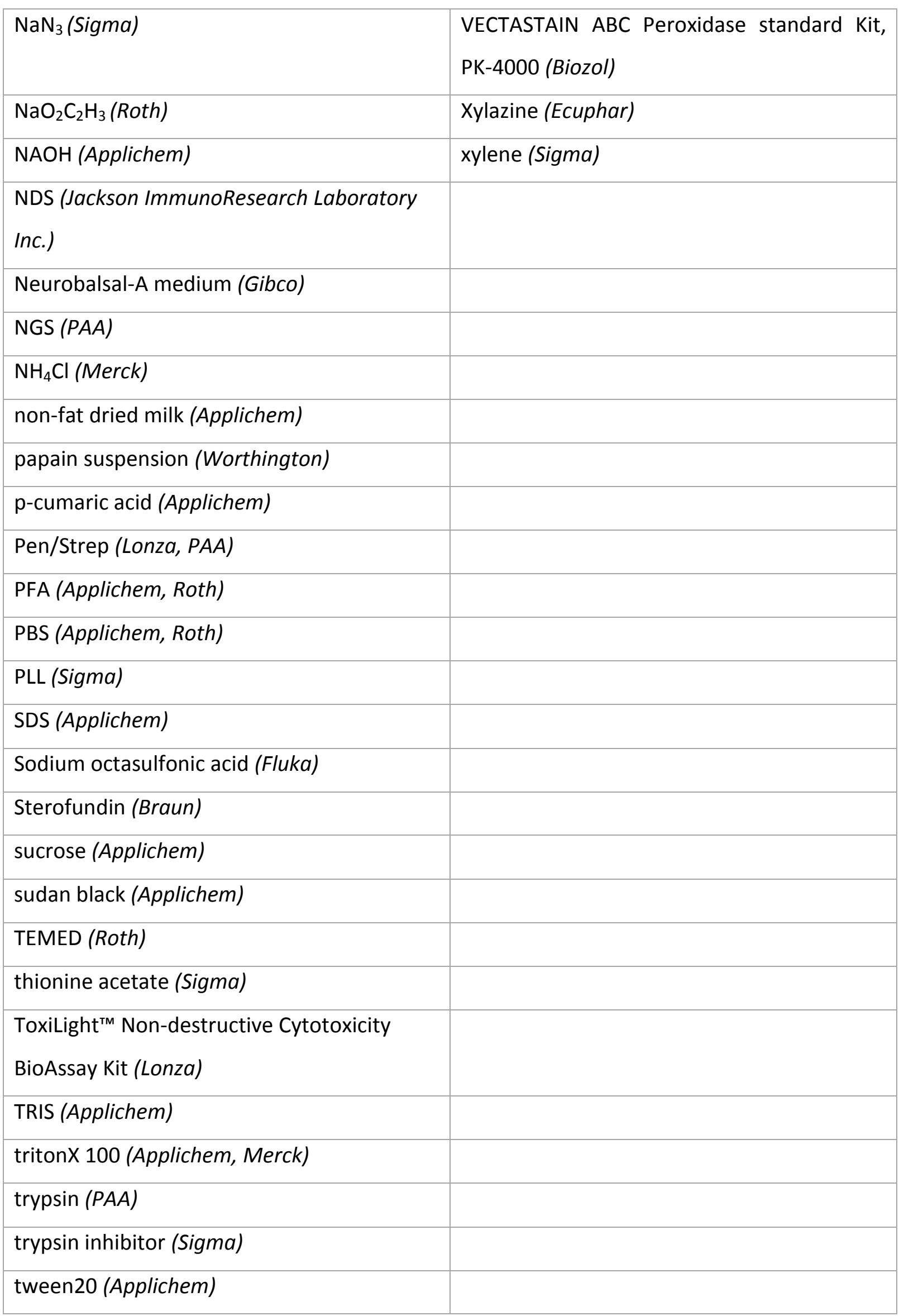




\subsubsection{Antibodies}

\section{Primary antibodies:}

Anti-ED1 (CD68): from mouse, monoclonal (Serotec)

Anti-glial fibrillary acidic protein (anti-GFAP): from rabbit, polyclonal (DAKO)

Anti-growth-associated protein-43 (anti-GAP-43): from rabbit, polyclonal (Abcam)

Anti-ionized calcium binding adapter molecule 1 (anti-lba1): from rabbit, polyclonal (Wako)

Anti-NeuN: from mouse, monoclonal (Millipore)

Anti-Rho kinase2 (anti-ROCK2): from goat, polyclonal (Santa Cruz)

Anti-synaptophysin: from rabbit, polyclonal (Merck Millipore); from guinea pig, polyclonal

(Synaptic Systems)

Anti-synaptotagmin1 (anti-syt1) Atto647N-labeled: from mouse, monoclonal (Synaptic Systems)

Anti-synaptotagmin1 -CypHer5E-labeled (CypHer): from mouse, monoclonal (Synaptic Systems)

Anti-tyrosine hydroxylase (anti-TH): from rabbit, polyclonal (Zytomed); from mouse, monoclonal (Sigma Aldrich); from goat, polyclonal (Abcam)

\section{Secondary antibodies:}

Anti-guinea pig Alexa 488: from donkey (Dianova)

Anti-mouse Cy2: from donkey (Dianova)

Anti-rabbit biotinylated: from goat (Dianova)

Anti-rabbit Dylight 488: from donkey (Dianova)

Anti-rabbit Alexa Fluor 488: from donkey (Dianova)

Anti-rabbit Cy3: from goat (Dianova)

Anti-rabbit Cy3: from donkey (Dianova)

Anti-rabbit Cy5: from goat, (Jackson ImmunoResearch Laboratory Inc.)

Anti-rabbit horse raddish peroxidase (HRP): from goat (Cell Signalling Technologies) 


\subsubsection{Equipment}

385 Stimulus Isolator (World Precision Intruments)

96 well micro test plate (Sarstedt)

12 well plate (Sarstedt)

A310 Accupulser Stimulator (World Precision Intruments)

Animal cages (IVC, Tecniplast)

Axioplan microscope equipped with a 16-bit grayscale CCD camera (Carl Zeiss Microimaging)

Axioplan microscope equipeed with MBF Q-imagin camera (Carl Zeiss Microimaging, MBF)

Bead mill homogenizer, Precellys $24^{\circledR}$ (Precellys by Peqlab)

Cage incubator (Okolab)

Catwalk XT gait analysis system (Noldus)

Camera Legria, HFM36 (Canon)

Ceramin beads $1.4 \mathrm{~mm}$ (Precellys by Peqlab)

Coulochem II electrochemical detector (ESA)

Cover slides $24 \times 60 \mathrm{~mm}$ (Menzel)

Cryostat, CM 3050 S (Leica)

Cryomatrix (Thermo Scientific)

Curix 60 Developer (Agfa)

Eclipse Ti-E microscope equipped with a HBO-100W Lamp and an IXON X3897 Andor Camera (Nikon Instruments)

Electrophoresis chamber (Bio-RAD)

Electrophoresis power supply (GE Healthcare)

Glass plates/Short plates (Bio-RAD)

Glass capillaries (World Precision Intruments)

Guard cell (ESA)

Menzelglass cover slides, diameter $18 \mathrm{~mm}$ (Menzel)

Micro4-based controller (World Precision Intruments)

Micro-centrifuge, 5415R (Eppendorf)

Micro injector, Nanoliter 2000 pump head (World Precision Intruments)

Mini-driller equipped with a $0.8 \mathrm{~mm}$ drill head (Dremel)

Mini Trans-Blot Cell setup (Bio-RAD)

Mouse jaw holder (David Kopf Instruments) 
Nitrocellulose transfer membrane (Applichem)

Rotarod for mice, model 47600 (UgoBasile)

Rotor type 45Ti (Beckman Coulter Inc.)

Sorvall Ultracentrifuge, Discovery 90SE (Hitachi GmbH)

Spacer plates (Bio-RAD)

Stereotactical frame (David Kopf Instruments)

SuperFrost Plus Microscope Slides 25x75 x 1.0 mm (Menzel)

TCS SP5 confocal microscope (Leica)

TCS SPE confocal microscope (Leica)

Wallac 1450 Micro beta TriLux-Luminometer (GMI)

Whatman gel blotting paper (GE Healthcare)

\subsubsection{Animals}

Male C57BI/6 mice (Charles River)

Postnatal wistar rats (Central Animal Facility of the UMG)

\subsubsection{Software}

AxioVision 4.6 (Carl Zeiss Microimaging)

CatWalk XT 10.0 (Noldus)

CorelDRAW X3 (Corel Corporation)

Cromeleon Chromatography Data System (Dionex. By Thermo Fisher Scientific GmbH)

GraphPad Prism Demo (GraphPad Software Inc.)

ImageJ $1.47 v$ (N.I.H.)

KyPlot 2.0 (KyensLab Incorporated)

LAS AF 2.4.1 (Leica)

MATLAB 7.5 (The MathWorks Inc.)

Sigma plot 10.0 (Systat Software $\mathrm{GmbH}$ )

Stereo Investigator9.0 (Micro Bright Field Inc) 


\subsubsection{Buffers and solutions}

Blocking and antibody solution for ICC: $2.5 \%$ BSA, $0.1 \%$ TritonX 100 in PBS

Blocking and antibody solution for IHC: $2.5 \%$ DS, $1 \%$ immunoglobulin free BSA, $0.1 \%$ TritonX 100 in PBS

Borate buffer: $100 \mathrm{mM}$; solution1: $19.7 \mathrm{~g} \mathrm{Na}_{2} \mathrm{~B}_{4} \mathrm{O}_{7} * 10 \mathrm{H}_{2} \mathrm{O}$ in $500 \mathrm{ml}$ distilled $\mathrm{H}_{2} \mathrm{O}$; solution2: $3.09 \mathrm{~g} \mathrm{H}_{3} \mathrm{BO}_{3}$ in $500 \mathrm{ml}$ distilled $\mathrm{H}_{2} \mathrm{O}$; adjust the $\mathrm{pH}$ of solution 2 with adequate amount of solution 1 to achieve a pH of 8.5.

DAPI solution: $1 \mu \mathrm{g} / \mathrm{ml}$ DAPI in PBS

ECL-1: $10 \mu \mathrm{l} / \mathrm{ml} 250 \mathrm{mM}$ luminol, $4.4 \mu \mathrm{l} / \mathrm{ml} 90 \mathrm{mM}$ p-cumaric acid, $100 \mu \mathrm{l} / \mathrm{ml}$ 1M Tris pH 8.5 in distilled $\mathrm{H}_{2} \mathrm{O}$

ECL-2: $0.9 \mu \mathrm{l} / \mathrm{ml} 30 \% \mathrm{H}_{2} \mathrm{O}_{2}, 100 \mu \mathrm{l} 1 \mathrm{M}$ Tris $\mathrm{pH} 8.5$ in distilled $\mathrm{H}_{2} \mathrm{O}$

Enzyme solution: $0.2 \mathrm{mg} / \mathrm{ml}$ cysteine, $2.0-2.5$ units of papain suspension/ml, $1 \mathrm{mM} \mathrm{CaCl}$, $500 \mu \mathrm{M}$ EDTA in DMEM

5-Fluoro-2'-desoxyuridine stock solution: $8.1 \mathrm{mM}$ 5-Fluoro-2'-desoxyuridine, $20.4 \mathrm{mM}$ uridine in DMEM

Gelelectrophoresis buffer: 192 mM Glycine, 0.1\% SDS, 25 mM Tris- $\mathrm{HCl}, \mathrm{pH} 8.3$

High salt PBS: $500 \mathrm{mM} \mathrm{NaCl}, 20 \mathrm{mM} \mathrm{Na}_{2} \mathrm{HPO}_{4}, \mathrm{pH} 7.4$

HPLC buffer: $6.973 \mathrm{~g} / \mathrm{L} \mathrm{NaO}_{2} \mathrm{C}_{2} \mathrm{H}_{3}, 7.365 \mathrm{~g} / \mathrm{L} \mathrm{C}_{6} \mathrm{H}_{8} \mathrm{O}_{7} * \mathrm{H}_{2} \mathrm{O}, 0.105 \mathrm{~g} / \mathrm{L}$ sodium octasulfonic acid, $0.048 \mathrm{~g}$ EDTA $\rightarrow \mathrm{pH} 4.3$ using $1 \mathrm{M} \mathrm{C}_{6} \mathrm{H}_{8} \mathrm{O}_{7}, 105 \mathrm{ml} / \mathrm{L} \mathrm{CH}_{4} \mathrm{O}$ in $800 \mathrm{ml} \mathrm{HPLC}$ water Inactivating solution: $2.5 \mathrm{mg} / \mathrm{ml}$ BSA, $2.5 \mathrm{mg} / \mathrm{ml}$ trypsin inhibitor, $10 \%$ FCS, in Neurobasal medium

Mowiol: $6 \mathrm{~g}$ Glycerin, $2.4 \mathrm{~g}$ mowiol, $6 \mathrm{ml} \mathrm{H} \mathrm{H}_{2} \mathrm{O}, 12 \mathrm{ml} 0.2 \mathrm{M}$ Tris $\mathrm{pH} 7.2$

Neurobasal medium: 0.2\% B27-supplement, 2 mM GlutaMAX, 1\% pen/strep in Neurobalsal-A medium

PBS: $9.5 \mathrm{mg} / \mathrm{ml}$ PBS in distilled $\mathrm{H}_{2} \mathrm{O}$

PFA solution (4\%): $40 \mathrm{mg} / \mathrm{ml}$ PFA, $9.5 \mathrm{mg} / \mathrm{ml} \mathrm{PBS,} 1-3$ drops $1 \mathrm{M} \mathrm{NaOH}$ in distilled $\mathrm{H}_{2} \mathrm{O}$

Plating medium: $10 \% \mathrm{HS}, 1 \%$ glutamine, $0.6 \mathrm{mg} / \mathrm{ml}$ glucose, in MEM

Running phase gel: $4.2 \mathrm{ml} 30 \%$ acrylamid bisacryl, $2.125 \mathrm{ml} 4 \mathrm{x}$ Tris $\mathrm{pH}$ 8.8, $2.125 \mathrm{ml}$ destilled $\mathrm{H}_{2} \mathrm{O}, 4.25 \mu \mathrm{l}$ TEMED, $42.5 \mu \mathrm{l}$ 10\% APS

Stacking phase gel: $0.65 \mathrm{ml} 30 \%$ acrylamid bisacryl, $1.25 \mathrm{ml}$ 4x Tris $\mathrm{pH}$ 6.8, $3.05 \mathrm{ml}$ destilled $\mathrm{H}_{2} \mathrm{O}, 5 \mu \mathrm{l}$ TEMED, $25 \mu \mathrm{l}$ 10\% APS

TBS: $10 \mathrm{mM}$ Tris $\mathrm{HCL}, 150 \mathrm{mM} \mathrm{NaCl}$ in destilled water 
TBS-T: 0.1\% Tween20 in TBS, pH 7.6

Transfer buffer: 192 mM Glycine, 20\% Methanol, 25 mM Tris-HCl, pH 8.3

Tris: $10 \mathrm{mM}$ Tris-buffered saline $\mathrm{pH} 8.0$

Tyrode buffer: $124 \mathrm{mM} \mathrm{NaCl}, 5 \mathrm{mM} \mathrm{KCl}, 30 \mathrm{mM}$ Glucose, $25 \mathrm{mM}$ HEPES in distilled $\mathrm{H}_{2} \mathrm{O}, \mathrm{pH}$ 7.4, afterwards: $1 \mathrm{mM} \mathrm{MgCl} 2,2 \mathrm{mM} \mathrm{CaCl} 2$

Washing solution for ICC: $2.5 \%$ BSA in PBS

\subsection{Methods}

\subsubsection{Human postmortem tissue}

\subsubsection{Characterization}

Human brain tissue for immunohistochemistry was obtained from the Department of Neuropathology and Prion Research of the Ludwig-Maximilians-University in Munich. Neuropathological diagnosis was based on the Consortium to Establish a Registry for Alzheimer's Disease (CERAD), the formation of neurofibrillary tangles (NFT) and Lewy body (LB) staging (McKeith et al., 2005; Heiko Braak et al., 2003). Brains arrived as formalin-fixed, paraffin-embedded $4 \mu \mathrm{m}$ sections placed on glass slides.

Brain sections from 7 Parkinson's disease patients (PD) (between 69 and 80 years old, average age $75.7+/-4.2$ years, 3 females and 4 males), 6 age-matched control subjects (amc) (between 67 and 87 years old, average age $78.3+/-8.5,2$ females and 4 males) and 5 young control subjects (yc) (between 47 and 60 years old, average age $55.4+/-5.2$ years, 5 males) were examined. From all individuals unilateral sections from the region of the midbrain containing the substantia nigra (SN) and the putaminal striatum (PU) were available. In two subjects there was no area of putamen available and therefore only the striatal caudate nucleus (NC) was included into the analysis. Patient case and tissue details are summarized in Table 2.1. 
Table 2.1: Summary of patient cases investigated. Abbreviations: amc = age-matched control; LBs = Lewy bodies; $\mathrm{ncl}$. = nucleus; NNP = no neuropathology; $\mathrm{PD}=$ Parkinson's disease; $\mathrm{PMI}=$ post-mortem interval; $\mathrm{SN}=$ substantia nigra; yc = young control.

$\begin{array}{ccccccccc}\begin{array}{c}\text { Case } \\ \text { No. }\end{array} & \text { Group } & \text { Age } & \text { Gender } & \text { Diagnosis } & \begin{array}{c}\text { LBs } \\ \text { (McKeith) }\end{array} & \begin{array}{c}\text { PM delay } \\ \text { (hrs) }\end{array} & \begin{array}{c}\text { sample } \\ \text { midbrain }\end{array} & \text { sample striatum } \\ 1 & \text { yc } & 58 & \text { male } & \text { NNP } & \text { no } & 8 & \text { SN } & \text { putamen } \\ 2 & \text { yc } & 60 & \text { male } & \text { NNP } & \text { no } & 11 & \text { SN } & \text { putamen } \\ 3 & \text { yc } & 58 & \text { male } & \text { NNP } & \text { no } & >24 & \text { SN } & \text { putamen } \\ 4 & \text { yc } & 54 & \text { male } & \text { NNP } & \text { no } & 9.5 & \text { SN } & \text { putamen } \\ 5 & \text { yc } & 47 & \text { male } & \text { NNP } & \text { no } & \text { na } & \text { SN } & \text { putamen } \\ 6 & \text { amc } & 86 & \text { female } & \text { NNP } & \text { no } & 20 & \text { SN } & \text { caudate ncl. } \\ 7 & \text { amc } & 71 & \text { male } & \text { NNP } & \text { no } & 23 & \text { SN } & \text { putamen } \\ 8 & \text { amc } & 75 & \text { male } & \text { NNP } & \text { no } & 27 & \text { SN } & \text { caudate ncl. } \\ 9 & \text { amc } & 67 & \text { male } & \text { NNP } & \text { no } & \text { na } & \text { SN } & \text { putamen } \\ 10 & \text { amc } & 87 & \text { male } & \text { NNP } & \text { no } & 48 & \text { SN } & \text { putamen } \\ 11 & \text { amc } & 84 & \text { female } & \text { NNP } & \text { no } & 22 & \text { SN } & \text { putamen } \\ 12 & \text { PD } & 71 & \text { male } & \text { PD } & \text { brainstem } & 60 & \text { SN } & \text { putamen } \\ 13 & \text { PD } & 77 & \text { female } & \text { PD } & \text { brainstem } & \text { na } & \text { SN } & \text { putamen } \\ 14 & \text { PD } & 77 & \text { male } & \text { PD } & \text { brainstem } & \text { na } & \text { SN } & \text { putamen } \\ 15 & \text { PD } & 80 & \text { female } & \text { PD-dementia } & \text { neocortical } & \text { na } & \text { SN } & \text { putamen } \\ 16 & \text { PD } & 76 & \text { male } & \text { PD-dementia } & \text { neocortical } & \text { na } & \text { SN } & \text { putamen } \\ 17 & \text { PD } & 69 & \text { female } & \text { PD } & \text { neocortical } & >48 & \text { SN } & \text { putamen } \\ 18 & \text { PD } & 80 & \text { male } & \text { DLB } & \text { neocortical } & 6 & \text { SN } & \text { putamen }\end{array}$

\subsubsection{Immunohistochemistry}

a) Fluorescence immunostaining

Single- or multi-fluorescent immunostainings were all performed according to the same protocol:

Paraffin-embedded sections were de-paraffinized for two times $10 \mathrm{~min}$ in xylene and rehydrated in ethanol solutions of decreasing concentration: $100 \%$ ethanol for 5 min, followed by $99 \%, 96 \%, 90 \%, 70 \%$ and $50 \%$ ethanol, each incubated for 5 min and stored in distilled water until next steps.

For antigen retrieval, slides were incubated in $50 \mathrm{mM}$ glycine ( $\mathrm{pH} 3.5)$ for $25 \mathrm{~min}$ at $90^{\circ} \mathrm{C}$ in water bath. After $1 \mathrm{~h}$ of cooling at room temperature (RT), sections were treated with sudan black B (0.3\% in $70 \%$ ethanol) for $5 \mathrm{~min}$ at RT to eliminate auto-fluorescence from lipids, rinsed 2 times for 5 min with PBS, followed by blocking and permeabilisation with $5 \%$ normal donkey serum (NDS), 1\% free bovine serum albumin (BSA, immunoglobulin and protease free) and $0.1 \%$ tritonX-100 in PBS for $1 \mathrm{~h}$ at RT. Immediately after blocking, 
incubation with primary antibodies was performed for single and co-stainings for $48 \mathrm{~h}$ at $4^{\circ} \mathrm{C}$ in 2.5\% NDS in PBS. The following antibodies were used: anti-tyrosine hydroxylase (anti-TH, rabbit, 1:1000; anti-TH, mouse, 1:500; anti-TH, goat, 1:500) anti-ROCK2 (goat, 1:50), antiGAP-43 (rabbit, 1:300), anti-synaptophysin (rabbit, 1:100), anti-glial fibrillary acidic protein (anti-GFAP, rabbit, 1:300), anti-ionized calcium binding adapter molecule1 (anti-lba1, rabbit, 1:300) and anti-CD68 (ED1, mouse, 1:50). Before incubation with the ED1 antibody, sections were pre-treated with $0.05 \%$ trypsin in PBS for $20 \mathrm{~min}$ at $37^{\circ} \mathrm{C}$ to achieve optimal antigen retrieval.

After incubation with primary antibodies, sections were rinsed 3 times for 5 min with PBS. Afterwards, all primary antibodies were detected by incubation with the appropriate species detecting secondary antibody for $1 \mathrm{~h}$ at RT. Fluorophore-conjugated secondary antibodies used were DyLight 488, Alexa Fluor 488, Cy2, Cy3 or Cy5 (all 1:250), respectively. For co-stainings the sections were incubated with the particular primary or secondary antibodies simultaneously. After additional rinsing with PBS, nuclei were stained with 4,6-diamidino-2-phenylindole (DAPI), rinsed again 3 times for $10 \mathrm{~min}$ and mounted with Mowiol $^{\circledR}$. Negative control staining was performed without incubation in primary antibodies asserting the appropriate detection of the secondary antibodies.

\section{b) Light stable immunostaining with 3,3'- diaminobenzidine (DAB)}

For counting of $\mathrm{TH}$-immunoreactive (TH-IR) cells in the SNpc and to investigate the TH-IR fiber density in the striatum, slides were de-paraffinized as described above (see 2.2.1.2), endogenous peroxidases were blocked for 3 min with $3 \% \mathrm{H}_{2} \mathrm{O}_{2}$ and rinsed 2 times for 5 min with Tris-base-saline (TBS). Sections were incubated with anti-TH antibody (from mouse, 1:70) for $48 \mathrm{~h}$ at $4^{\circ} \mathrm{C}$ followed by rinsing 3 times for $5 \mathrm{~min}$ with TBS. Treatment with a secondary biotinylated anti-rabbit antibody (1:200), at RT for $2 \mathrm{~h}$ followed. After washing again 3 times for 5 min in TBS, incubation with VECTASTAIN ABC Peroxidase standard Kit (PK4000) for $2 \mathrm{~h}$ at RT was performed to enhance detection signal. Finally sections were stained with DAB (DAB peroxidase substrate Kit) for $15 \mathrm{~min}$ and mounted with DPX. 


\subsubsection{Stereological quantification of substantia nigra dopaminergic neurons and evaluation of the striatal dopaminergic fiber density in human brain sections}

The number of TH-IR neurons in the human substantia nigra (SN) was assessed unilaterally using stereological methodology. One section through the SN was analyzed per case using Stereo Investigator software and a Zeiss microscope. The area of the SN was manually outlined using a $2.5 x$ objective and a point grid was overlaid onto each section constituting areas of the counting frames (counting frame 500x500 $\mu \mathrm{m}$ ). Immunostained cells were counted by the optical fractionator method (10x objective) and the total number of TH-IR cells in the unilateral SN was estimated per slice by the software. Stereological counts were performed by a blinded investigator.

To analyze the TH-IR fiber density, sections were acquired with a 10x objective using the Stereo Investigator software which assembles single acquired images into one picture via the Virtual Slice ${ }^{\circledR}$ module. TH-IR was detected by measuring the mean grey value using the ImageJ software.

\subsubsection{Image acquisition and analysis of protein expression in the human SNpc and striatum}

Brain sections were imaged with a Zeiss Axioplan microscope equipped with a 16-bit grayscale CCD camera and AxioVision software using a 63x objective. Micrographs were taken at 15-20 randomly chosen visual fields per SNpc or putamen (caudate nucleus respectively), containing TH-IR cells or striatal neuropil. For detailed analysis of the fluorescent signal of ROCK2, GAP-43 or synaptophysin staining, an appropriate area of the TH-IR soma or TH-IR neurite, as well as of the adjacent neuropil was chosen to measure the mean grey value of the respective immunofluorescence using the freehand selection from ImageJ (see Figure 2.1). 


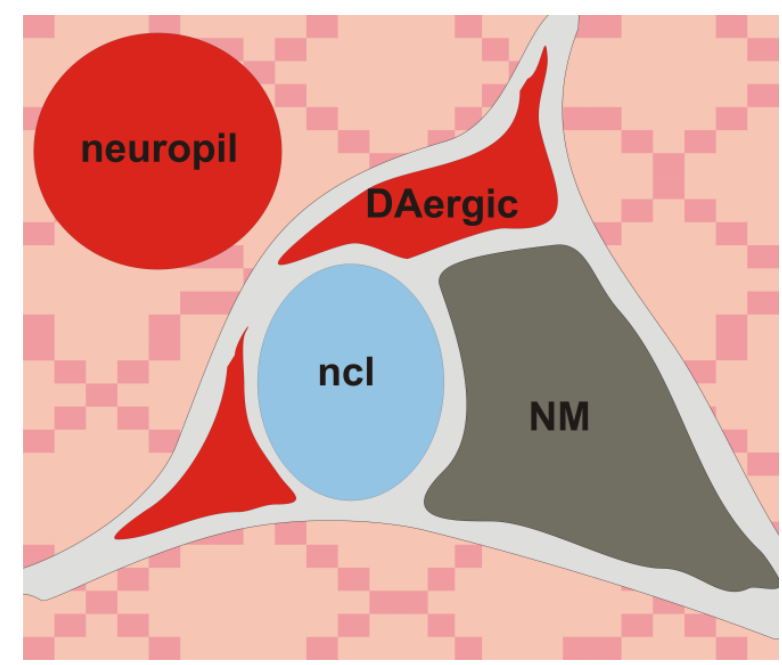

Figure 2.1: Illustration of method to measure immunofluorescence in certain areas of the midbrain. Dopaminergic cells (DAergic) were identified by TH staining and intracellular measurement was performed in areas (red) not containing neuromelanin (NM) or the nucleus (ncl). For evaluation of immunoreactivity in the neuropil, a randomly chosen area was analyzed (red circle), containing no TH-IR structures or other nuclei.

To allocate enhanced ROCK2 expression to specific cell types, 20 images (98.41x $98.41 \mu \mathrm{m}$ ) from the SNpc or the striatum, respectively, were acquired randomly using Leica Microsystems TCS SP5 confocal microscope with a $63 x$ oil immersion objective (kindly provided by the Max-Planck-Institute for biophysical chemistry, Göttingen) in order to correlate ROCK2-IR to astrocytes or to Iba1-IR microglia. Therefore co-labeled GFAP- and ROCK2-, or Iba1- and ROCK2-IR cells were quantified using ImageJ cell counter plugin.

In order to distinguish between phagocytic active and resting ROCK2-positive microglia the number of ED1-/ROCK2 co-labeled cells was quantified with ImageJ cell counter plugin on 5 randomly chosen micrographs $(400 \times 530 \mu \mathrm{m})$ taken with the AxioVision 4.6 MosaiX module with a $63 x$ objective.

\subsubsection{The 6-OHDA mouse model of PD and intracranial injection of AAV.shRNA}

As animal model for PD we chose the 6-OHDA mouse model, because it addresses best the focus of our interests in order to mimic the nigrostriatal neurodegeneration in PD. A unilateral injection of the neurotoxin 6-OHDA was applied in the striatal putamen and simultaneously the shRNA containing vector was injected into the ipsilateral SN using a stereotactical frame. 


\subsubsection{Animals}

The surgeries of mice were performed under the grant approval 11-0554 and were carried out regarding to the regulations of the local animal research council and legislation of the State of Lower Saxony (Braunschweig, Germany).

For the experiments, 8 weeks old male C57BI/6 mice were housed in groups of five in individually ventilated cages under a $12 \mathrm{~h}$ light/12h dark cycle with free access to food and water. Mice were pre-treated with metamizol $(1.5 \mathrm{mg} / \mathrm{ml})$ in drinking water 3 days prior to the surgeries to achieve optimal analgesic conditions during and after the operation.

\subsubsection{Virus}

The viruses were kindly provided by Prof. Dr. Uwe Michel and Dr. Jan C. Koch (both Department of Neurology, UMG) and have been produced as described before (Koch et al., 2014; Michel et al., 2005). Shortly, adeno-associated viral vectors (AAV) co-expressing a short-hairpin RNA (shRNA) under the control of the H1-promoter and the fluorophore dsRed under the control of the neuron-specific human synapsin promoter were produced. The sequences of the shRNA primers were as follows (in bold: sequence of the respective siRNAsense and -antisense strand, in italics: sequence of the hairpin turn):

ROCK2-shRNA forward primer:

$5^{\prime}-$

GATCCCCTGCAAAGTTTATTATGATATACTTCCTGTCATATATCATAATAAACTTTGCATTTTTGGAA A-3'.

ROCK2-shRNA reverse primer:

$5^{\prime}-$

AGCTTTTCCAAAAATGCAAAGTTTATTATGATATATGACAGGAAGTATATCATAATAAACTTTGCAG GG-3'.

The vectors used for virus production were pAAV-9(5)hSyn-DsRed-H1-ROCK2-shRNA containing the $\mathrm{H} 1$ promotor for the shRNA and the hSyn promoter for the fluorophore dsRed (Figure 2.2-A). The respective control vector, expressing an shRNA against EGFP and the fluorophore dsRed was pAAV-9(5)hSyn-DsRed-H1-EGFP-shRNA (Figure 2.2-B). For in vivo experiments, the virus production was performed using the serotype AAV2 as it is best 
established for this paradigm, targeting primarily neurons (Löw \& Aebischer, 2012; Tenenbaum, 2004).

\subsubsection{Surgery preparations/equipment}

Stereotactic injections of 6-OHDA were performed according to the protocol of (Alvarez-Fischer et al., 2008; Tatenhorst et al., 2014)). In detail, before starting the surgery, the micro injector was prepared by filling a self-pulled glass capillary with around $3 \mu \mathrm{l}$ mineral oil, connecting it to the micro injector and withdrawing a $500 \mathrm{nl}$ air bubble to achieve separation of oil and substance to inject. $4 \mu \mathrm{g}$ of 6-OHDA were freshly dissolved in a volume of $2 \mu \mathrm{l} 0.2 \%$ L-ascorbic acid (L-AA) in PBS and the 6-OHDA substance or the virus solution, respectively, was withdrawn into the capillary in amounts of $1.5-2.5 \mu l$ of substance with the help of a micro4-based controller.

\subsubsection{Injection of 6-OHDA and the AAV.shRNA}

Mice were anesthetized by a mixture of ketamine $(200 \mathrm{mg} / \mathrm{kg}$ bodyweight) and xylazine $\left(10 \mathrm{mg} / \mathrm{kg}\right.$ body weight) in Sterofundin ${ }^{\circledR}$ (infusion solution). Eyes were protected with eye ointment (bepanthene) and mice were head fixed by ear bars and jaw holder in a stereotaxic frame (Figure 2.2-C). The skin covering the skull was disinfected and cut with a scalpel through the midline, starting between the eyes until the occipital bone exposing bregma and lambda. The skin was moved to the sites by small hooks and bregma was calibrated. With a mini-driller equipped with a $0.8 \mathrm{~mm}$ drill head two minimal trepanations at the positions of the coordinates for the right striatum (coordinates relative to Bregma: anterior-posterior (AP) $+0.04 \mathrm{~cm}$; medio-lateral $(\mathrm{ML})-0.18 \mathrm{~cm}$; dorso-ventral (DV) $-0.35 \mathrm{~cm}$, Figure 2.2-D) and the ipsilateral SN (coordinates relative to Bregma: AP $-0.29 \mathrm{~cm}, \mathrm{ML}-0.12$ $\mathrm{cm}, \mathrm{DV}-0.45 \mathrm{~cm}$, Figure 2.2-E) accordingto the mouse brain atlas (Paxinos \& Watson, 2001) and the coordinates from Alvarez-Fisher and colleagues (2008) were performed. The capillary was moved to the particular depth and the respective substance was injected in a rate of $0.5 \mu \mathrm{l} / \mathrm{min}$ (Figure 2.2-C). Afterwards the needle was left in place for $5 \mathrm{~min}$ before retraction to prevent reflux via the injection tract. 
After removing the injection capillary, mice were unhinged from the stereotactic frame and the skin lesion was closed with $10 \mu \mathrm{l}$ of histoacrylic glue. Subcutaneous injection of $1 \mathrm{ml}$ pre-warmed Sterofundin ${ }^{\circledR}$ was administered and mice were warmed with a $37^{\circ} \mathrm{C}$ heating pad until they woke up. In the following days mice were treated with metamizol (minimum 3 consecutive days), fed with mashed food and monitored for vitality.
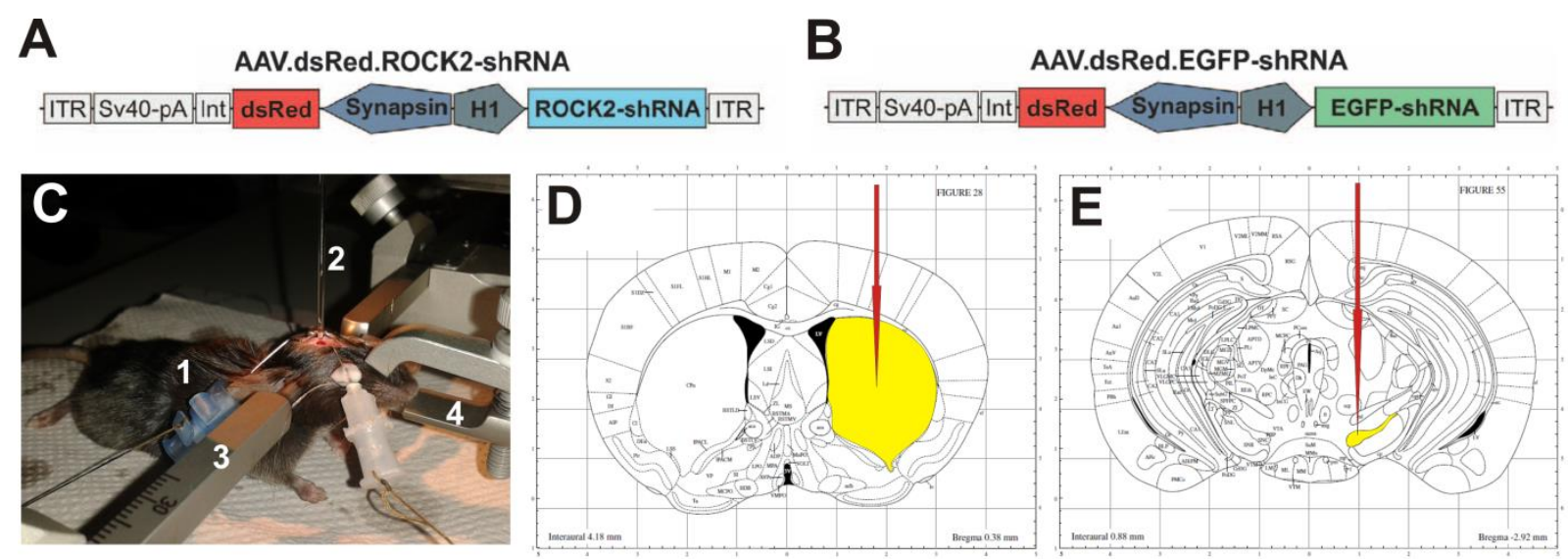

Figure 2.2: Illustration of the striatal 6-OHDA mouse model of PD and treatment with ROCK-shRNA into the SNpc. The vector map of the H1-controled AAV.ROCK2-shRNA is shown in A and the respective control map, expressing a shRNA against EGFP, is depicted in B. Mice (1) are head-fixed with ear bars (3) and a jaw holder (4) in a stereotaxic frame. The glass capillary (2) is moved down to the respective position to inject the substance. $\mathbf{D}$ and $\mathbf{E}$ are illustrating the sections from the coordinates of the putaminal striatum (yellow area in $\mathbf{D}$ ) and the midbrain with the SNpc (yellow area in E) both from Paxinos \& Watson mouse brain atlas (Paxinos and Watson, 2001).

\subsubsection{Animal group classification}

All animals received intracranial injections of $2 \mu \mathrm{l}$ substance (either 6-OHDA or the vehicle L-AA) into the right striatum and simultaneously $1 \mu$ l substance (either AAV.shRNA or the vehicle PBS) into the ipsilateral SN. In order to achieve optimal effects of shRNA mediated ROCK2 downregulation in dopaminergic SN neurons, two different virus titers were employed: One group of animals was injected with a high virus titer of $1.0 \times 10^{8}$ transforming units (TU) in $1 \mu \mathrm{l}$ PBS (in the following named as 6-OHDA/ROCK2-shRNA1.0) and the second group received AAV in a titer of $0.25 \times 10^{8} \mathrm{TU} / \mu \mathrm{l}$ (named as 6-OHDA/ROCK2shRNA0.25). Vehicle control groups were treated with $2 \mu \mathrm{l}$ of $0.2 \%$ L-AA into the striatum and $1 \mu$ PBS into the ipsilateral SN (named as LAA/PBS). Lesion control animals were injected with $2 \mu \mathrm{l}$ 6-OHDA into the right striatum and $1 \mu \mathrm{l}$ PBS into the SN (named as 6-OHDA/PBS). 
Virus control animals received injections of $2 \mu$ 6-OHDA into striatum and $1 \mu \mathrm{l}$ AAV.dsRed.EGFP-shRNA in the high titer of $1.0 \mathrm{x} 10^{8} \mathrm{TU} / \mu \mathrm{l}$ into the SN (named as 6-OHDA/control-shRNA). Both AAV.ROCK2-shRNA groups were injected with $1 \mu$ l of the virus solution into the SN and simultaneously with $2 \mu \mathrm{l}$ 6-OHDA into the ipsilateral striatum.

\subsubsection{Behavior analyses}

To assess motor behavior, four different tests, the apomorphine turning test, the rotarod assessment, the cylinder rearing test and the Catwalk XT gait analysis, were performed two and four weeks after lesion. The animal numbers in the respective test and respective time point are summarized in table 2.2 .

Table 2.2: Number of animals tested in motor performance at two different time points.

\begin{tabular}{|c|c|c|c|c|c|}
\hline Time point & Group & $\begin{array}{l}\text { Apomorphine } \\
\text { turning test }\end{array}$ & $\begin{array}{l}\text { Cylinder } \\
\text { rearing test }\end{array}$ & Rotarod & $\begin{array}{c}\text { Catwalk } \\
\text { gait analysis }\end{array}$ \\
\hline \multirow{5}{*}{$\begin{array}{c}2 \text { weeks } \\
\text { after } \\
\text { injections }\end{array}$} & $L A A / P B S$ & 10 & 10 & 10 & 10 \\
\hline & 6-OHDA/PBS & 8 & 10 & 10 & 10 \\
\hline & 6-OHDA/control-shRNA & 10 & 10 & 10 & 10 \\
\hline & 6-OHDA/ROCK2-shRNA0.25 & 12 & 12 & 12 & 12 \\
\hline & 6-OHDA/ROCK2-shRNA1.0 & 11 & 11 & 11 & 11 \\
\hline \multirow{5}{*}{$\begin{array}{c}4 \text { weeks } \\
\text { after } \\
\text { injections }\end{array}$} & $L A A / P B S$ & 10 & 10 & 10 & 10 \\
\hline & 6-OHDA/PBS & 8 & 8 & 8 & 8 \\
\hline & 6-OHDA/control-shRNA & 10 & 10 & 10 & 10 \\
\hline & 6-OHDA/ROCK2-shRNA0.25 & 12 & 12 & 12 & 12 \\
\hline & 6-OHDA/ROCK2-shRNA1.0 & 11 & 11 & 11 & 11 \\
\hline
\end{tabular}

\section{a) The apomorphine turning test}

This behavioural test was applied to monitor the efficacy of the unilateral 6-OHDA lesion in the treatment groups and to determine potential recovery effects in turning behaviour after AAV.ROCK2-shRNA treatment. Application of the dopamine agonist apomorphine results in a rotation of mice to the contralateral (ctl) side induced by a hyperstimulation of compensatory upregulated D2-receptors on striatal postsynapses (Ungerstedt, 1971). Mice received a subcutaneous injection of apomorphine $0.5 \mathrm{mg} / \mathrm{kg}$ body weight in $0.9 \%$ saline, were placed in round arena of $20 \mathrm{~cm}$ in diameter and turning behaviour was monitored ten minutes after injection for 20 min by videotaping with a video 
camera from above. The test was performed at two and four weeks after 6-OHDA lesion/virus injection and total turns to the ctl as well as to the ipsilateral (ipl) side were counted manually.

b) The cylinder rearing test

The cylinder rearing test was employed to use natural mice exploration activity to assess potential unilateral unbalance in dopamine levels in the brain which may result in unequal forelimb usage after 6-OHDA lesion. Each mouse was placed into a plexiglass cylinder of $25 \mathrm{~cm}$ in height and $11.5 \mathrm{~cm}$ in diameter (Figure 2.3-A). During the test the explorative behaviour was videotaped from the front and forelimb use of the first contact against the glass wall while rearing was recorded for $5 \mathrm{~min}$. A 90 degrees angulated mirror was arranged behind the cylinder to enable observation of forelimb usage even if the mouse turned away from the camera (Figure 2.3-A). Rating criteria were "right" and "left" when the animal used the respective forelimb singly to touch the cylinder during a rear, "both" when the animal used both left and right forelimb simultaneously to touch the cylinder during a rear or "free" when the animal reared up the body without touching the cylinder wall. Recorded movies were manually analyzed. Statistical analysis pictured the percentage of "both", "right", left" or "free" movements of all movements recorded during observation time.

\section{c) Rotarod performance test}

The rotarod test was performed at two and four weeks after cerebral injections. Mice were placed on the rotarod and were forced to motor activity by the rotating rod spinning with an accelerating speed (5 to $40 \mathrm{rpm}$ within $5 \mathrm{~min}$ ). The rod featured 5 separated sites rendering the possibility to let 5 mice perform the test simultaneously (Figure 2.3-B). Each trial stopped when a mouse fell down and thereby activated a lever, which automatically stopped the time, or when 5 min motor performance was completed. All mice were pretrained three times on the rotarod on every second day before cerebral injections. The final test was performed in three sessions with an intertrial interval of $30 \mathrm{~min}$ to avoid stress and 
prostration. Each motor performance was quantified by measuring the time mice stayed on the rod and the average value was compared between the groups.

\section{d) Catwalk gait analysis}

To monitor changes in gait performance of mice injected with 6-OHDA and the AAV.shRNA, the Catwalk XT gait analysis system was used. Animals were placed on a glass plate walkway of $4 \mathrm{~cm}$ width and videotaped from below with a high speed digital camera, connected to a computer equipped with the Catwalk software (Figure 2.3-C). The combination of a red illuminated ceiling and the green illuminated glass plate enable the software to automatically detect the animal paw or body prints on the glass plate.

Detection settings to achieve optimal distinction between paw prints (Figure 2.3-D) and background noise were chosen as follows: camera gain 24.41, intensity threshold 0.27 and maximal allowed speed variation of an animal during one run was set to $60 \%$. Three compliant runs per animal were recorded, parameters for each single paw print (Figure 2.3-E) were evaluated and the means for different parameters were compared between the groups. 

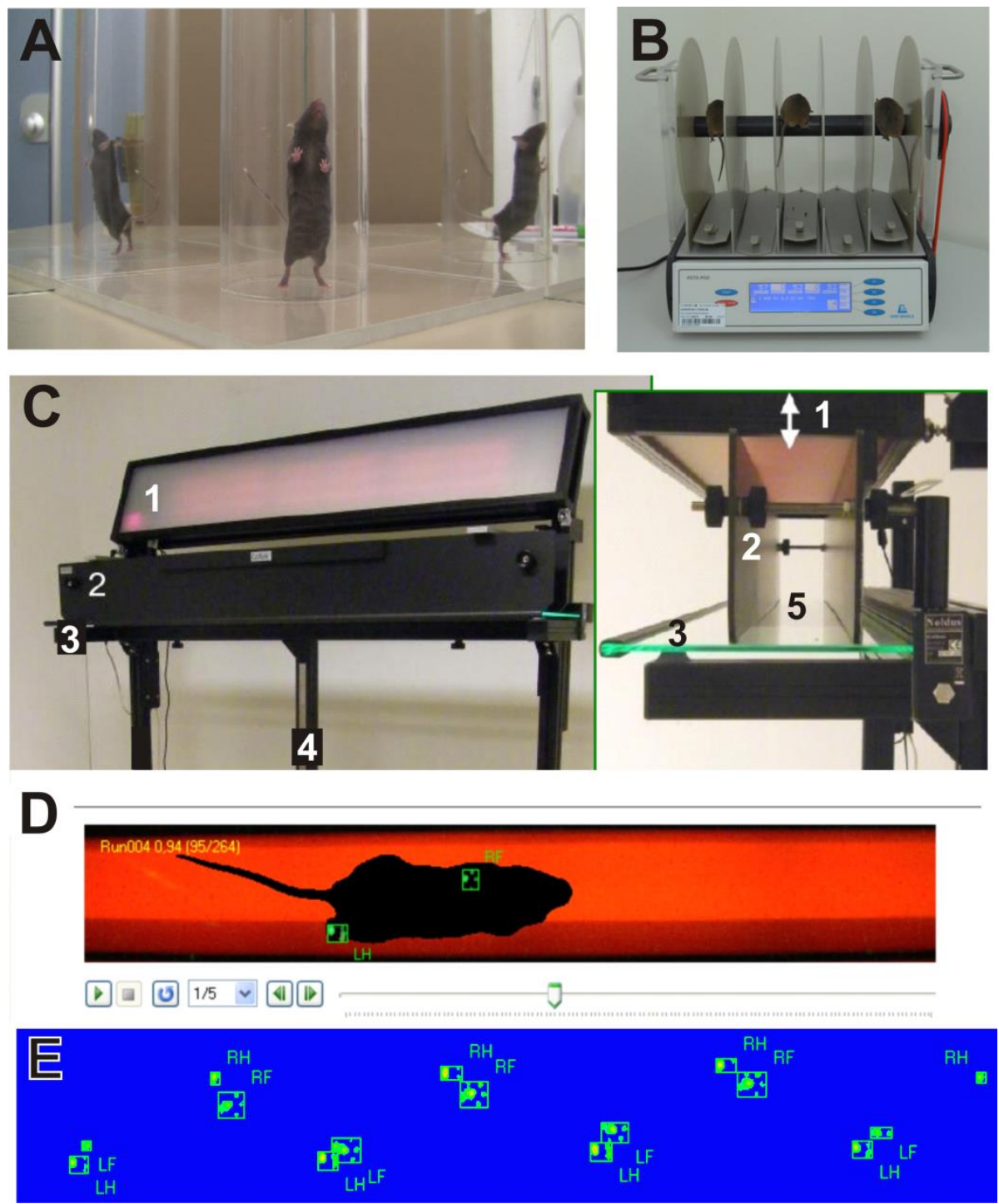

Figure 2.3: Overview of the experimental setup for behavioural motor tests. The cylinder rearing test is illustrated in A. Mice could be observed during rearing from all sides with the help of a mirror, which was arranged behind the cylinder. The rotarod test (B) allows the observation of motor endurance of 5 mice simultaneously running on the rod. Subtle motor changes could be precisely measured with the Catwalk gait analysis (C-E). The hardware compounds are depicted in C from the front (left image) and from the side (right image) with 1: red illuminated ceiling, 2: corridor walls, 3: green illuminated glass walkway, 4: camera position and 5: the walkway tunnel. In $\mathbf{D}$ a single frame of the recordings is illustrated, showing a mouse running through the walkway. The footprints are detected by the camera and classified for the whole run as LH: left hind, LF: left front, RH: right hind, RF: right front (E). 


\subsubsection{Animal euthanasia and tissue processing}

Four weeks after the 6-OHDA lesion and the injection of viral vectors animals were sacrificed and either perfused for immunohistochemistry (IHC) or brains were freshly prepared for neurochemical analysis.

\subsubsection{Transcardial perfusion}

For immunohistochemical investigations of protein expression levels, the transcardial perfusion method was performed to clear the vasculature from blood, fix the ultra-structure of the tissue and preserve protein conformation. Animals were deeply anaesthetized with $\mathrm{CO}_{2}$ inhalation, supinely fixed on a rack and the abdominal cavity was opened. The diaphragm was carefully intersected and the ribs were bilaterally cut to open the thorax. The pericardium was removed and the still beating heart was perforated in the left ventricle with a cannula connected with a tube to a syringe filled with ice-cold PBS. By increasing pressure from the syringe, the PBS was forced to enter the ventricle and further into the aorta. Setting a small cut in the right atrium allowed the blood to be voided and enabled the PBS to perfuse the whole body including the brain. Successful blood cleaning was indicated by colour changing of the liver from red to yellow. After perfusion of approximately $50 \mathrm{ml}$ of ice-cold PBS, the fluid was replaced with $50 \mathrm{ml}$ of $4 \%$ paraformaldehyde (PFA) in PBS. The brain was removed and post-fixed in 4\% PFA for two days, then transferred into $30 \%$ sucrose in PBS for three days to dehydrate the tissue. Brains were frozen at $-80^{\circ} \mathrm{C}$ until cryosectioning.

\subsubsection{Cryosectioning and IHC of mouse brains}

The still frozen mouse brains were embedded into cryomatrix and the region of the striatum (Bregma AP +1.18 to -0.70) and the midbrain containing the area of the SN (Bregma AP -2.18 to $-4.04 \mathrm{~mm}$ ) were coronally cryosectioned using a Leica cryostat resulting in $30 \mu \mathrm{m}$ sections and stored in $0.1 \%$ sodium azide/PBS at $4^{\circ} \mathrm{C}$ until staining.

For evaluation of dopaminergic cell numbers in the $\mathrm{SN}$, every fourth section was stained using a free-floating method. After rinsing the sections 3 times for 5 min with Trisbuffered saline (TBS) endogenous peroxidases were quenched by incubating sections in $40 \%$ 
methanol and $1 \% \mathrm{H}_{2} \mathrm{O}_{2}$ in TBS for 15 min. After washing again in TBS unspecific antibody binding was blocked by $5 \%$ NGS in TBS for $1 \mathrm{~h}$ at RT. Incubation with an anti-TH primary antibody (anti-TH, rabbit) diluted in $2 \% \mathrm{NGS} / \mathrm{TBS}$ was performed for $48 \mathrm{~h}$ at $4^{\circ} \mathrm{C}$.

After washing 3 times for $5 \mathrm{~min}$ in TBS, sections were treated with a secondary biotinylated anti-rabbit antibody in TBS at RT for $2 \mathrm{~h}$. Incubation with VECTASTAIN ABC Peroxidase standard Kit for $2 \mathrm{~h}$ at RT was performed to enhance signal detection. Finally, sections were rinsed again 3 times for $5 \mathrm{~min}$ in TBS and visualized with the chromogen DAB (DAB peroxidase substrate Kit) for $5 \mathrm{~min}$. The staining reaction was stopped with distilled $\mathrm{H}_{2} \mathrm{O}$ and sections were collected on SuperFrost Plus microscope slides. After drying for around 3-5 days at RT, a Nissl staining was performed as follows: sections were rehydrated for $5 \mathrm{~min}$ in distilled $\mathrm{H}_{2} \mathrm{O}$ and incubated in $1 \%$ thionine acetate for $7 \mathrm{~min}$. After rinsing the slides again in distilled $\mathrm{H}_{2} \mathrm{O}$ for $2 \mathrm{~min}$, dehydration of the tissue was performed by incubation in ascending alcoholic solutions of 70\%, 90\%, 95\% ethanol each for 2 minutes, followed by 5 $\min$ in $100 \%$ isopropanol and subsequent incubation in xylene 3 times for 5 min. Finally, moist sections were mounted with DPX.

For striatal TH-density analysis, every fourth section was collected on microscope slides and stained according to the same protocol described above (see nigral TH-IHC), but the antibody solutions were put on the top of the slide. The DAB staining was performed with the Elite $A B C$ Kit with nickel intensification and sections were mounted with DPX without Nissl staining.

To demonstrate the cell type specificity of the synapsin-promoter-driven dsRed expression, nigral sections were stained with the free floating method as follows: sections were rinsed 3 times for 5 min with PBS and blocked with 5\% NGS in 0.1\% PBS-TritonX-100 for 30 minutes at RT. Subsequently, samples were incubated with anti-neuronal nuclei (NeuN) (1:1000), anti-GFAP (1:500) or anti-lba1 antibody (1:500) for $24 \mathrm{~h}$ at $4^{\circ} \mathrm{C}$. After 3 rinses in PBS for $5 \mathrm{~min}$, sections were incubated with Cy2-conjugated antibody (1:250) for $1 \mathrm{~h}$ at RT. Finally, sections were DAPI stained and mounted with Mowiol ${ }^{\circledR}$. 


\subsection{Neurochemical analysis of dopamine and dopamine metabolites in the striatum of 6-0HDA lesioned in mice}

To specify the functional impairment of dopaminergic terminals and synapses in the striatum at four weeks after the 6-OHDA intoxication, one cohort of mice was sacrificed by cervical dislocation, to ensure immediate processing of brain tissue without anaesthetic additives. The brain was removed from the skull and cut along the midline resulting in two hemispheres. The right striatum was dissected on ice, transferred into cryovials (micro tube $2 \mathrm{ml}$ ), which were previously filled with $0.6-0.8 \mathrm{~g}$ of Precellys sceramin beads $1.4 \mathrm{~mm}$ and snap frozen with liquid nitrogen until further processing. To degrade enzymatic action $50 \mu \mathrm{l}$ of $0.1 \mathrm{M} \mathrm{HClO}_{4}$ per mg of striatal tissue were added and samples were homogenized with a bead mill homogenizer. Subsequently vials were centrifuged in a microcentrifuge at 10,000 rounds per minute ( $9300 \mathrm{~g}$ ) for $5 \mathrm{~min}$, supernatant was transferred into a new reaction tube and again centrifuged at $13,000 \mathrm{rpm}$ for $10 \mathrm{~min}$ at $4^{\circ} \mathrm{C}$. To measure the amount of dopamine (DA), homovanillic acid (HVA) and 3,4-dihydroxyphenylacetic acid (DOPAC) $50 \mu \mathrm{l}$ of supernatant were transferred into HPLC vials.

The HPLC system was composed of a Guard cell, which was kept at $600 \mathrm{mV}$ to oxidize impurities in the eluent, a C18 reverse-phase HR-80 catecholamine column for catecholamine extraction and an ESA Coulochem II electrochemical detector equipped with a $5011 \mathrm{~A}$ analytic model detector $(\mathrm{E} 1=50 \mathrm{mV}, \mathrm{E} 2=400 \mathrm{mV}$ ). For measurements, the samples were loaded to the HPLC autosampler, which was cooled down before to $6^{\circ} \mathrm{C}$ and the system injected $20 \mu \mathrm{l}$ of each sample to the column. The mobile phase (HPLC buffer, filtered and degased) was running with a flow rate of $0.4 \mathrm{ml} / \mathrm{min}$. The HPLC buffer prevented changes in $\mathrm{pH}$ during the mobile phase, which could affect the retention time of the analysed molecules and result in changes in the chromatogram. To accurately determine catecholamine amounts, dopamine, HVA and DOPAC standards were run every 15 samples. The standards were prepared from $10 \mu \mathrm{M}$ stocks by dilution with $0.1 \mathrm{M} \mathrm{HClO}_{4}$ to $0.0,0.15,0.30$ and $1.5 \mu \mathrm{M}$. Data collection and processing was done with a Chromeleon Chromatography Data System by integrating the areas under the specific peak curves. Data was displayed as ng per mg wet tissue. Groups analysed were as follows: LAA/PBS with $n=10 ; 6-O H D A / P B S$ with $n=8$; 6-OHDA/control-shRNA with $\mathrm{n}=10 ; 6-\mathrm{OHDA} /$ ROCK2-shRNA0.25 with $\mathrm{n}=10$ and 6-OHDA/ROCK2-shRNA1.0 with $n=11$. 


\subsubsection{Stereological quantification of SNpc neurons in mice}

The number of TH-positive neurons and Nissl positive cells in the SN was determined by stereological counting using Stereo Investigator software from DAB immunostained brain sections. Every $4^{\text {th }}$ section through the SN was analyzed by two blinded investigators. At $2.5 \mathrm{x}$ objective magnification the SN was outlined manually and the TH-IR and Nissl-positive cells were counted at 40x objective magnification using the optical fractionator method: cells were identified in counting frames sized $50 \times 50 \mu \mathrm{m}$ and the total number of immunolabeled cells was calculated by the optical fractionator method for the whole unilateral SNpc per animal. The evaluation of Nissl cells was performed to confirm loss of neurons rather than mere down-regulation of TH-expression. Values represent averaged assessments in the ipsilateral SN of the hemisphere with dual intracerebral injections. Groups analyzed were as follows: LAA/PBS with $n=4,6-O H D A / P B S$ with $n=7,6-O H D A / c o n t r o l-s h R N A$ with $n=5$, 6-OHDA/ROCK2-shRNA0.25 with $\mathrm{n}=4$ and 6-OHDA/ROCK2shRNA1.0 with $\mathrm{n}=5$ after four weeks.

\subsubsection{Striatal density measurement in mouse striata}

To assess the dopaminergic degeneration in the lesioned striatum, the TH-IR was measured four weeks after cerebral injections. Immunostained sections of each animal were acquired using the Virtual Slice module of Stereo Investigator software described above (see 2.2.1.3.), the mean gray value of striatum and cortex were measured using ImageJ freehand selection, followed by image processing with background subtraction. Five sections per animal around the lesion site were analyzed and the mean TH-IR-signal was calculated in relation to the TH-signal of the contralateral striatum (lesioned hemisphere/unlesioned hemisphere in \%). Groups included were as follows: LAA/PBS with $n=5,6-O H D A / P B S$ with $\mathrm{n}=7$, 6-OHDA/control-shRNA with $\mathrm{n}=5,6-\mathrm{OHDA} /$ ROCK2-shRNA0.25 with $\mathrm{n}=4$ and 6-OHDA/ROCK2-shRNA1.0 with $\mathrm{n}=5$ after four weeks. 


\subsubsection{In vitro inhibition of ROCK2 in primary neuron cell culture}

Primary hippocampal cell cultures were employed to investigate synaptic vesicle dynamics under treatment of the ROCK2 inhibitor fasudil. To establish a mature neuronal network with numerous synaptic compositions, cells have to be cultured up to 12-14 days in vitro (DIV). To generate optimal physiological conditions for preserving the vitality of cells, it is important to coat the culture plates or coverslips with attachment factors and provide an adequately nutritive culture medium. Donor animals were supplied by the central animal facility of the UMG and experiments were performed in the European Neuroscience Institute (ENI) Göttingen in cooperation with Prof. Silvio Rizzoli.

\subsubsection{Coating of culture plates and preparation of coverslips}

For stimulation experiments, hippocampal neurons were cultured onto glass coverslips (diameter: $18 \mathrm{~mm}$ ), which first were cleaned by shaking in $200 \mathrm{mM} \mathrm{HNO}_{3}$ on a lab shaker over night (ON). Afterwards the coverslips were rinsed several times with distilled water and finally in $70 \%$ ethanol. Before being placed into 12 well-culture plates, the ethanol was flamed and all further procedures were performed under sterile conditions. For coating, the coverslips were transferred into the well plates and incubated with $1 \mathrm{ml} 1 \mathrm{mg} / \mathrm{ml}$ Poly-Llysine (PLL) per well in borate buffer $\mathrm{ON}$ in an incubator at $37^{\circ} \mathrm{C}$ with $5 \% \mathrm{CO}_{2}$ and $95 \%$ humidity. After rinsing 4 times with sterile distilled water, $1 \mathrm{ml}$ plating medium was filled in each well and plates were kept in the incubator for 2 days.

\subsubsection{Tissue dissection}

To obtain primary hippocampal neurons, young Wistar rats (postnatal day 0-2) were killed by decapitation, the brains were exposed, the hippocampi from both hemispheres were dissected and the meninges were removed. The hippocampi were pooled together in ice-cold HBSS, transferred into the pre-warmed enzyme solution and kept slowly rotating at $37^{\circ} \mathrm{C}$ for $60 \mathrm{~min}$. Later, the enzyme solution was replaced by pre-warmed inactivating solution for $15 \mathrm{~min}$ and rinsed with pre-warmed Neurobasal medium. Using a handmade fire-polished Pasteur pipette, cells were gently triturated for dissociation in fresh Neurobasal medium, then counted with a Neubauer counting chamber and seeded in a density of 
around 50,000 - 100,000 cells/well into $1 \mathrm{ml}$ plating medium. Neurons were kept in incubator at $37^{\circ} \mathrm{C}$ with $5 \% \mathrm{CO}_{2}$ and $95 \%$ humidity for $1 \mathrm{~h}$ and then the whole medium was replaced by $1.5 \mathrm{ml} /$ well pre-warmed Neurobasal-culture medium. A partial Neurobasal medium change $(500 \mu \mathrm{l})$ was performed on DIV1-2 and after 5 DIV $20 \mu \mathrm{l}$ 5-Fluoro-2'deoxyuridine stock solution was added to each well to inhibit the proliferation of glia cells in culture.

For western blotting, cells were seeded without coverslips into culture plates with a density of 500,000 cells/well.

\subsubsection{In vitro treatment with the ROCK2 inhibitor fasudil}

The concentration of $20 \mu \mathrm{M}$ fasudil was chosen, because it had the most pronounced effect on neuronal survival and neurite outgrowth in primary dopaminergic midbrain cultures in previous studies (Tönges et al., 2011). Primary hippocampal neurons were treated with $20 \mu \mathrm{M}$ fasudil at DIV10 by adding $20 \mu \mathrm{l}$ of $1 \mathrm{mM}$ fasudil solution into each well containing $1 \mathrm{ml}$ medium and gently mixed. On DIV12 the whole medium was replaced by fresh pre-warmed medium containing new $20 \mu \mathrm{M}$ fasudil. Experiments were performed on DIV14-15.

\subsubsection{Toxilight ${ }^{\mathrm{TM}}$ cell toxicity assay}

To exclude toxic effects on the neurons after fasudil treatment, we used the ToxiLight $^{\mathrm{TM}}$ non-destructive cytotoxicity Bio Assay Kit from Lonza. The Kit quantitatively measures the release of adenylate kinase (AK) from dying cells by adding the AK substrate ADP, which is converted by AK to ATP. Luminescence resulted from the reaction of ATP and luciferin, so that the emitted light is linearly related to AK concentration. For the ToxiLight ${ }^{\mathrm{TM}}$ assay, $100 \mu \mathrm{l}$ of culture medium supernatant were collected from each well on DIV14-15 and $50 \mu \mathrm{l}$ were transferred in duplications into a 96 well plate. For background correction, fresh medium was measured in 2 wells. To each sample $50 \mu \mathrm{l}$ of the AK detection reagent were added and the measurement was performed after 5 min incubation time. Luminescence was acquired by a Wallac TriLux-Luminometer, single values were averaged and the background was subtracted. Data was generated from 3 independent experiments. 


\subsubsection{Electric cell stimulation}

To investigate the effects of long term ROCK2 inhibition on synaptic vesicle exo- and endocytosis, two experimental setups were used according to the protocols from Prof. Silvio Rizzoli (see also Wilhelm et al., 2010): in one approach the cells were fixed after the experiment to enable immunocytochemistry (ICC) and evaluation of stimulation-induced events, in particular by identifying synapses with co-staining of synaptophysin. In the other experiment, a life imaging depicted the vesicle dynamics during and directly after stimulation by real time acquisition.

To perform experiments with fixable readout, coverslips with cultured neurons on the surface were gently rinsed in pre-warmed physiological tyrode buffer and placed into a custom-made platinum wire field stimulation chamber (Figure 2.4-A). To allow for antibody incorporation into presynapses, cells were incubated in pre-warmed tyrode buffer containing an anti-synaptotagmin1 (syt1) antibody conjugated with Atto ${ }^{\circledR} 647 \mathrm{~N}$ (1:100) for 30 seconds and electrical field stimulation was applied through the platinum wires $(8 \mathrm{~mm}$ distance) which reached into the antibody solution close to the cell surface. By strong stimulation of neurons with field pulses at $20 \mathrm{~Hz}$ for $30 \mathrm{~s}$ at $100 \mathrm{~mA}$ with a stimulus isolator and an Accupulser stimulator, at RT synaptic vesicles underwent membrane fusion and transmitter release. Thereby the intravesicular domain of the syt1 transmembrane protein was exposed to the extracellular antibody solution and provided thereby the antigen for the syt-antibody. During vesicle recycling and endocytosis, the fluorophore-conjugated syt-antibody is endocytosed into the neuron (Figure 2.4-B). To guarantee the uptake of all endocytosed vesicles, which underwent the transmitter release before, the coverslips were left in the antibody solution for 6 minutes from the start of stimulation. Afterwards, cells were rinsed in ice-cold tyrode buffer and fixed in 4\% PFA in PBS on ice for 10 min and further 30 min at RT.

To generate a negative or unstimulated control, coverslips were incubated in ice-cold tyrode buffer containing the Atto ${ }^{\circledR} 647 \mathrm{~N}$ conjugated syt-antibody (1:100) and $3 \mu \mathrm{M}$ tetrodotoxin (TTX) to selectively block voltage depended sodium channels and to minimize spontaneous action potentials. After rinsing cells again in ice-cold tyrode buffer, they were fixed as described above.

For life imaging, incubation with syt1-CypHer5E-labeld antibody (1:100 in Neurobasal medium) was performed for $1-1.5 \mathrm{~h}$ at $37^{\circ} \mathrm{C}$ in an incubator. CypHer is a $\mathrm{pH}$-sensitive cyanine 
dye that is maximally fluorescent at a $\mathrm{pH}$ of 5.5 and non-fluorescent at $\mathrm{pH}$ 7.4. During incubation, it is internalized into the neurons by endocytosis from spontaneous bursting activity of hippocampal neurons (Pimashkin et al., 2011), depicting the highest fluorescent signal from the luminal acidic milieu of vesicles. After rinsing the coverslips with pre-warmed tyrode buffer, they were fixed in the stimulation chamber covered with tyrode buffer and attached to an inverted Nikon Eclipse Ti-E microscope equipped with a plan 60x oil immersion objective and cage incubator system to maintain a constant temperature of $37^{\circ} \mathrm{C}$ and the $\mathrm{CO}_{2}$ level at 5\%. The filter set used was: Cy5/CypHer ET/EX: 620/60. The acquisition was started once the stimulation chamber was placed into the microscope, recording images every two seconds with an exposure time of 200-300 ms. After $1 \mathrm{~min}$ acquisition of the resting state, neurons were stimulated with $20 \mathrm{~Hz}$ for $30 \mathrm{~s}$ at $100 \mathrm{~mA}$ and images were recorded up to 6 min to allow total recycling of exocytosed synaptic vesicles.

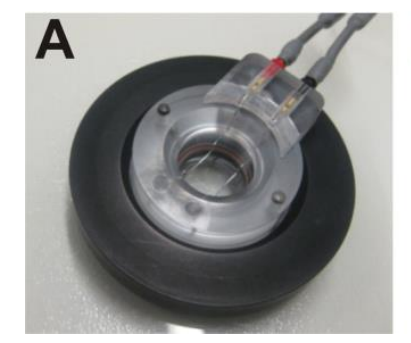

B stim

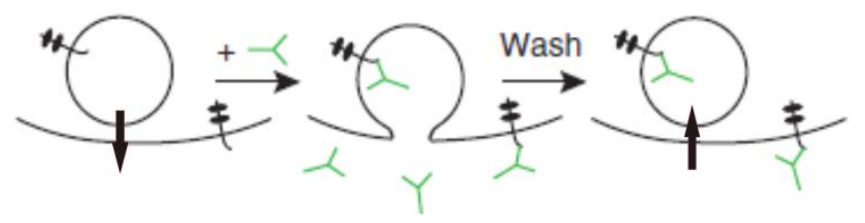

Figure 2.4: Experimental setup for in vitro cell stimulation. In $A$ the stimulation chamber is pictured. In the middle of the chamber, the coverslip is placed and the platinum wires are fixed above. In B a scheme (modified from Wilhelm et al., 2010) of the antibody coupling procedure is illustrated: as reaction to a stimulus, vesicles fuse with the plasma membrane, thereby present the intraluminal antigene to the antibody solution and incorporate the antibody during endocytosis into the cell.

\subsubsection{Immunocytochemistry}

To identify synapses in culture, a co-staining with the synaptic marker synaptophysin was performed. After fixation, cells were shortly rinsed with PBS and incubated in $100 \mathrm{mM}$ $\mathrm{NH}_{4} \mathrm{Cl}$ in PBS for 20 min at RT to quench free aldehydes from PFA. For blocking of unspecific bindings, coverslips were incubated with 2.5\% BSA and 1\% TritonX-100 3 times for 5 min on shaker at RT. Afterwards incubation with primary anti-synaptophysin (anti-synphy, 1:500) antibody in blocking solution was performed for $1 \mathrm{~h}$ at RT and then rinsed 3 times for 5 min 
with blocking solution. Thereafter, the coverslips were incubated with secondary anti-guinea pig-Alexa Fluor488 antibody (1:200) in blocking solution for $1 \mathrm{~h}$ at RT. Coverslips were washed with $2.5 \%$ BSA, followed by rinsing with a high salt PBS solution and finally with PBS, all 3 times for $5 \mathrm{~min}$ at RT. Coverslips were mounted with Mowiol and imaged with Leica Microsystems TCS SPE confocal microscope (kindly provided by the Max-Planck-Institute for biophysical chemistry, Göttingen) with a 100x oil immersion objective.

\subsubsection{Image processing and analysis of in vitro experiments}

To assess the amount of synaptophysin and syt1 co-localized synaptic boutons on fixed hippocampal neurons, all images were acquired with the same laser intensity and smart gain for all conditions at 15-20 randomly chosen visual fields from the coverslip.

All image and data analysis for in vitro experiments were performed with customwritten routines from Prof. Silvio Rizzoli using MATLAB 7.5. Confocal acquired images were exported into RAW data tiff.-files and processed by a MATLAB script, which subtracted the mean background of a defined area in each picture and calculated the number of co-localized pixels generated from syt1-Atto ${ }^{\circledR} 647 \mathrm{~N}$ - and synphy-immunostaining. The mean pixel values, representing the synaptic activity, were compared between the groups.

Movies generated from life imaging were processed with ImageJ and the plugin "intensity vs. time monitor" to save the pixel information over time as txt.-files. MATLAB was programmed to calculate a bleaching correction for each movie to obtain only fluorescent shifts indicated by stimulation and changing of $\mathrm{pH}$ during endo- and exocytosis. Furthermore, the fluorescent information for each time frame from the movies was averaged to output one fluorescent value per time point resulting in 181 values depicting a curve of fluorescent change after stimulation. From this curve single vesicle kinetic events were investigated. The measured fluorescent signal decreased during exocytosis and increased during endocytosis due to the $\mathrm{pH}$ of the luminal milieu similar to exponential curves. Thus, with the help of Sigma Plot software, a computation of an exponential fit was performed that describes best the averaged curves per condition. The resulting curve relationships were best fitted with 3 parameter exponential decay (exocytosis) or exponential rise to maximum (endocytosis) functions. To calculate the vesicle kinetics the time constant $\tau$ was identified, which describes the time an exponential decay needs to 
reach around $36.8 \%(1 / \mathrm{e})$ of the initial value. $\tau$ also describes the time an exponential rise needs to reach around $63.2 \%$ of the maximum value. Using these calculations, the vesicle kinetics for exocytosis and reuptake were compared between the control and the fasudil treated group and depicted as means per condition.

\subsubsection{Western blot analysis}

To assess molecular changes in the synaptic G- and F-actin ratio after fasudil treatment, a western blot analysis for G-actin protein amounts were performed by using a G-actin/F-actin in vivo assay kit that provided all needed solutions and antibodies to perform the cell lysis and western blotting according to manufacturer's instructions.

\section{a) Cell harvesting}

For generating samples, medium was removed and pre-warmed LAS2 buffer (containing lysis and F-actin stabilization buffer, 100 mM ATP stock, 100 x protease inhibitor cocktail, all from actin/F-actin in vivo assay kit) was added to each well, cells were harvested with cell scraper and transferred to a $1.5 \mathrm{ml}$ Eppendorf tube on ice. Cells were homogenized by gently triturating with a $200 \mu \mathrm{l}$ pipette and incubated for $10 \mathrm{~min}$ at $37^{\circ} \mathrm{C}$. Then $100 \mu \mathrm{l}$ of each sample were centrifuged at $350 \mathrm{xg}$ at RT for 5 min to pellet unbroken cells or tissue debris. The supernatant was transferred to ultracentrifuge tubes and centrifuged at 100,000 x g ( 32785.901 rpm, Rotor type $45 \mathrm{Ti}$ ) at $37^{\circ} \mathrm{C}$ for $1 \mathrm{~h}$ to pellet F-actin and keep the G-actin in the supernatant. Subsequently, the supernatant was gently transferred into fresh tubes, 100 $\mu l$ of F-actin depolymerization buffer (G-actin/F-actin in vivo assay kit) was added to each pellet and incubated for $1 \mathrm{~h}$ on ice and samples were mixed every $15 \mathrm{~min}$ to allow complete F-actin depolymerization to G-actin. To denature proteins, $25 \mu$ I SDS buffer (G-actin/F-actin in vivo assay kit) were added to each pellet as well as to each supernatant sample and mixed well. G-actin quantification was performed by SDS-page.

b) SDS-polyacrylamide gel electrophoresis (SDS-page)

The principle of SDS-page is based on the separation of proteins under the influence of electric field within the gel matrix. SDS-page was performed on a $5 \%$ stacking phase gel 
and a $12 \%$ resolving phase gel. The rack with polymerized gels was placed into electrophoresis chamber, filled with ice-cold electrophoresis buffer and $10 \mu$ of each sample were loaded in the gel columns. To assess G-actin protein amounts in the loaded samples, Gactin protein standards (G-actin/F-actin in vivo assay kit) were additionally run through the gel in amounts of 10, 20, $50 \mathrm{ng}$ G-actin in $15 \mu \mathrm{l}$ depolimerization/SDS buffer. To define the molecular weight of loaded proteins, a molecular weight marker (dual protein standard) was loaded and separated in parallel. G-actin was expected at $43 \mathrm{kDa}$. The electrophoresis was run with an electrophoresis power supply first with a voltage of $50 \mathrm{~V}$ to allow samples to enter the stacking phase gel without smearing for around $15 \mathrm{~min}$. Then the voltage was raised to $100 \mathrm{~V}$ and let stable until the smallest proteins were reaching the bottom of the gel.

\section{c) Immunoblotting and band visualisation}

To transfer the proteins to a nitrocellulose membrane a Mini Trans-Blot Cell setup was used. Loading the blotting cassette was performed by packing the gel next to the membrane between two sheets of Whatman filter paper and fiber pads which were previously soaked in transfer buffer. The cassette was placed in the transfer buffer-filled Mini Trans-Blot Cell in the direction that proteins could be transferred from cathode (from gel) to anode (to membrane). The blotting was performed with $25 \mathrm{~V}$ ON at $4{ }^{\circ} \mathrm{C}$. After protein transfer, the membrane was blocked with 5\% non-fat milk in TBS-T for 30-60 min at RT. The membrane was rinsed 3 times for 10 min and incubation with the G-actin antibody (rabbit, polyclonal, 1:500, G-actin/F-actin in vivo assay kit) was performed in $1 \%$ non-fat milk in TBS$\mathrm{T}$ for $1 \mathrm{~h}$ at RT, followed by rinsing the membrane 3 times for $10 \mathrm{~min}$ with TBS-T. The treatment with secondary anti-rabbit antibody coupled to horseradish peroxidase (HRP) $(1: 1000)$ in $1 \%$ non-fat milk in TBS-T was performed for $0.5-1 \mathrm{~h}$ at RT. Membrane was then rinsed 5 times for $10 \mathrm{~min}$ with TBS-T and finally treated with chemiluminescent reagents to detect the bands of $\mathrm{G}$-actin at $43 \mathrm{kDa}$. For the chemiluminescent reaction the solution of ECL-1 was mixed in equal parts with ECL-2 and added to the membrane for a minimum of 1 min allowing the antibody coupled-HRP to react with luminol. Afterwards membranes were exposed to autoradiography films (Hyperfilm ${ }^{T M} \mathrm{ECL}^{\mathrm{TM}}$ ) in different time intervals of 10-60 s with the help of an autoradiography cassette. The films were developed with a Curix 60 Developer. 


\subsubsection{Evaluation of G-/F-actin ratio in hippocampal cultures}

To evaluate the ratio of $\mathrm{G}$-actin from the supernatant and F-actin from the pellet G-actin bands from autoradiography, films were scanned and band intensities were analyzed with the ImageJ gel analysis method. Finally, the G-actin amount of supernatant divided by G-actin amount of the pellet revealed the ratio between G-/F-actin in the sample and was compared between the groups.

\subsubsection{Statistics}

Statistical analyses were conducted using Kyplot software. We assumed the data obtained being normally distributed, therefore applying parametric statistical methods, which are considered valid for most biological samples. Data from human tissue analysis was compared between all 3 groups (yc vs. amc, amc vs. PD or yc vs. PD) using one-way ANOVA with Tukey Kramer post-hoc test. For the in vivo mouse experiments, group comparisons were always referred to the adequate control (e.g. LAA/PBS vs. 6-OHDA/PBS; 6-OHDA/control-shRNA vs. OHDA/ROCK2-shRNA), thus one-way ANOVA followed by Dunnett's test were used. In vitro experiments were statistically evaluated by one-way ANOVA followed by Dunnett's test. The applied statistical test and number of experiments is indicated in each figure legend. Data are presented as mean \pm standard error of the mean (SEM). Differences were considered significant with $p<0.05 \quad\left({ }^{*} p<0.05 ; \quad *^{*} p<0.01\right.$; $* * * p<0.001)$. 


\section{Results}

\subsection{Analysing the human brain: Is there a regeneration failure in PD?}

To answer the question how and to what extent intrinsic regenerative capacities in the nigrostriatal system of Parkinson's disease (PD) cases may be inhibited, we performed an immunohistochemical analysis of human brain tissue to elucidate if the expression levels of certain growth regulating proteins are altered. To estimate the individual disease progression from available patient cases, well-known features specifying anatomical characteristics of PD were evaluated by immunohistochemistry as well.

\subsubsection{Immunohistochemical characterization of patient cases}

The different structural constitution of human midbrain and striatal brain sections of three example patient cases -one from each group- is visualized by tyrosine hydroxylase ( $\mathrm{TH}-$ ) immunostaining in Figure 3.1/1-A. The upper panel illustrates the putamen (PU) and the caudate nucleus $(\mathrm{NC})$, divided by the internal capsule $(\mathrm{Cl})$ in exemplary cases of a young control (yc), an age-matched control (amc) and a PD patient. The NC is adjacent to the lateral ventricle and the PU is separated by the external capsule from the insular cortex. These adjacent structures were not present in all sections and therefore not further investigated. The decrease in $\mathrm{TH}$-immunoreactivity (TH-IR) in the striatum was obviously detectable in PD cases compared to yc (Figure 3.1/1-A, upper panel, right micrograph), although there was no significant decrease in TH-IR fiber amount by evaluation of the mean TH-IR in the PU from all groups. Nevertheless a strong trend towards less TH fiber density was ascertainable in PD cases $(46.05 \pm 2.33 \mathrm{TH}-\mathrm{IR}$ signal) compared to both control groups (yc $=68.19 \pm 8.15 \mathrm{TH}-\mathrm{IR}$ signal, amc $=58.06 \pm 7.77 \mathrm{TH}-\mathrm{IR}$ signal) (Figure 3.1/1-B).

The lower panel in Figure 3.1.1-A shows brain sections through the midbrain of yc, amc and PD cases. The substantia nigra (SN) and the ventral tegmental area (VTA) appeared from the TH-staining in a brown stripe of dopaminergic neurons between the red nucleus (NR) and the pedunculus cerebri (PC). In PD cases the TH-IR in the SN was strongly decreased, which was already detectable from overview micrographs (Figure 3.1/1-A, lower panel, right micrograph) caused by the loss of TH-IR cells (Figure 3.1/1-A, lower panel, 
detail). The relative number of dopaminergic neurons per individual was estimated by stereological counting of the TH-IR cells in one unilateral section through the SN per case. The counting of cells with the stereo investigator system allows the automatic selection of defined counting frames with same size and inter-frame distance for all cases to calculate the number of counted cells in the marked area. The quantified TH-IR cell numbers confirmed the significant loss of around 65\% dopaminergic neurons in PD patients with a mean of $3.7 \pm 1.33$ cells per counting frame (Figure 3.1/1-C, declaring one outlier) compared to age-matched ( $\mathrm{amc}=11.84 \pm 1.06$ cells per counting frame) and young controls $(\mathrm{yc}=10.11$ \pm 0.98 cells per counting frame). The other midbrain areas were not further investigated.
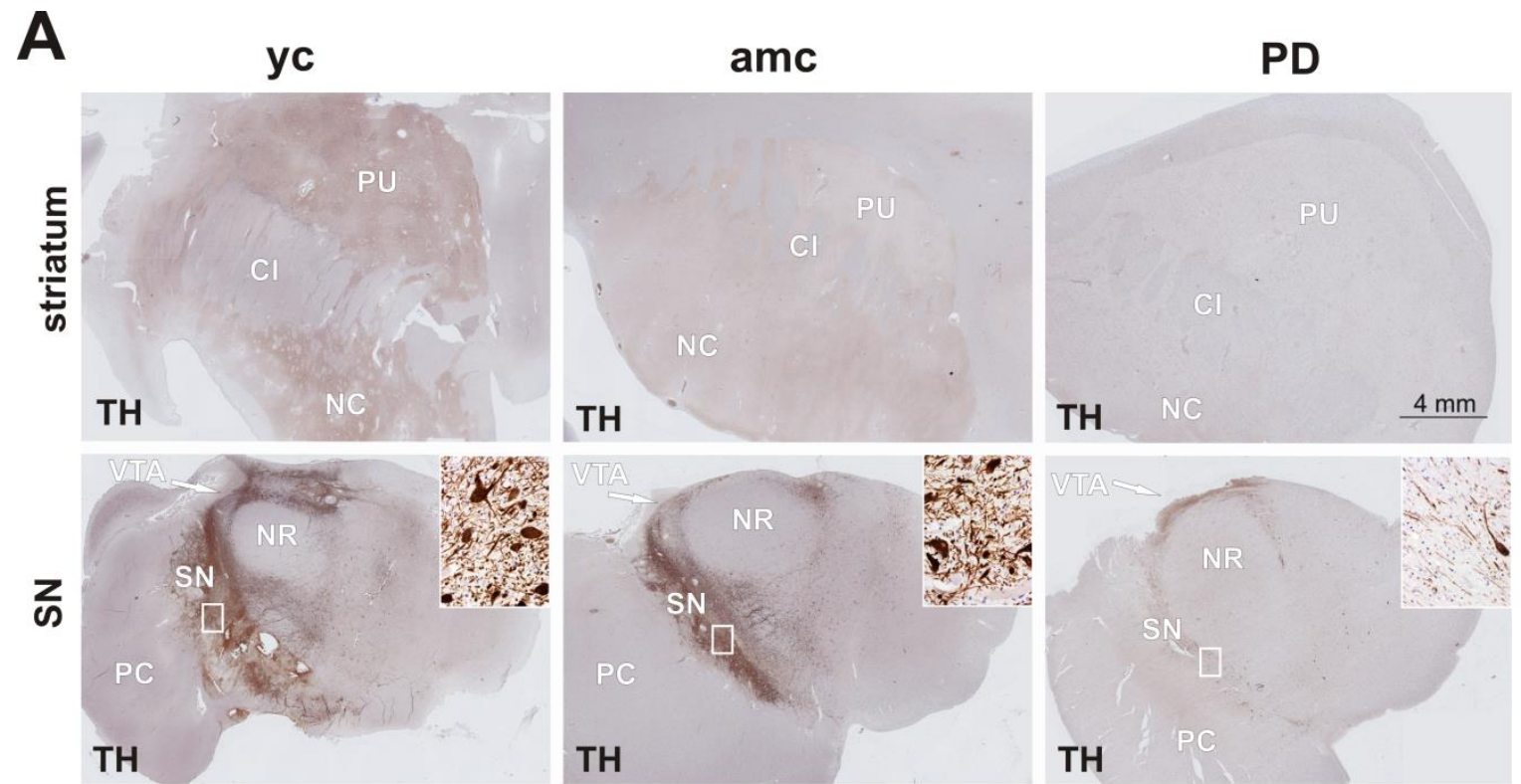

B

\section{Dopaminergic fibers} in striatum

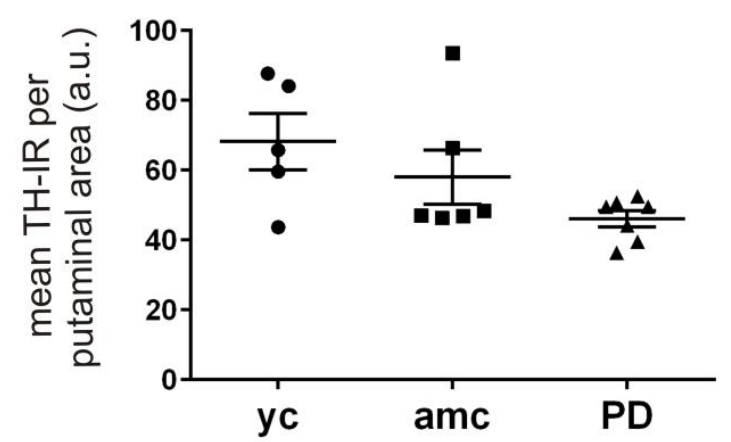

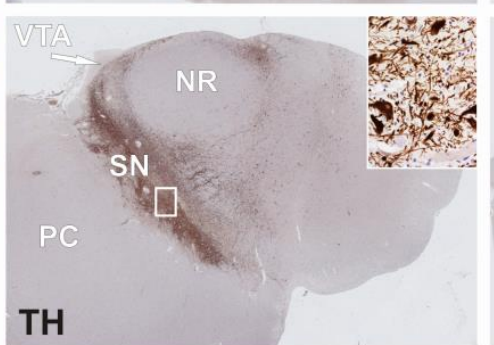

TH

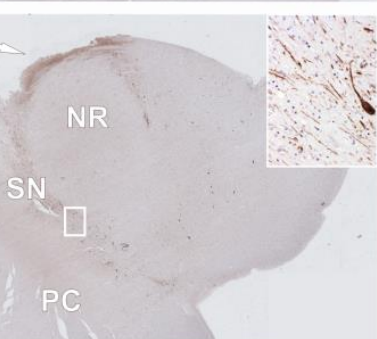

TH
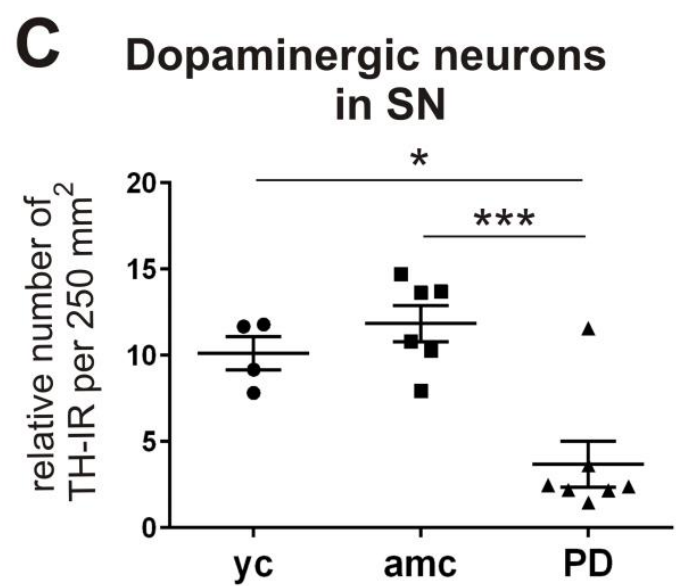

Figure 3.1/1: Immunohistochemical characterization of human brain sections. Micrographs show representative TH-DAB-immunostainings and illustrate the investigated areas of the striatum (A, upper panel) and substantia nigra (A, lower panel, inset: magnification from SNpc) in young controls (yc), age-matched 
controls (amc) and PD cases (PD). $\mathrm{Cl}=$ internal capsule, $\mathrm{NC}=$ caudate nucleus, $\mathrm{NR}=$ red nucleus, $\mathrm{PC}=$ pedunculus cerebri, $\mathrm{PU}=$ putamen, $\mathrm{SN}=$ substantia nigra. Quantification of dopaminergic fiber density in putaminal striatum (B, n.s.; yc: $n=5$; amc: $n=6$; PD: $n=7$ ) and cell number in SNpc (C, yc: $n=4 ;$ amc: $n=6 ; P D: n=7)$. Data is given in averaged values for each case, bars represent means per group $\pm \mathrm{SEM}$. ${ }^{*} \mathrm{P}<0.05 ; * * * \mathrm{P}<0.001$ (One-way ANOVA with Tukey Kramer test for independent comparison between groups).

In addition to the extent of dopaminergic degeneration, the degree of astrocytosis and microgliosis in the substantia nigra pars compacta (SNpc) and striatum was evaluated. Therefore astrocytes were identified by fluorescence immunohistochemistry against GFAP. Concomitant with the dopaminergic degeneration in the nigrostriatal system, there is an increase of GFAP-immunoreactivity (GFAP-IR) in the putaminal striatum. This augmentation in astrocytic markers was strongly detectable in PD cases and also in the age-matched control group, while in contrast, the yc showed less GFAP-IR signal (Figure 3.1/2-A, upper panel). The quantification of GFAP-IR cells confirmed these findings resulting in statistically significant differences in GFAP-IR structures between the groups $(y c=8.286 \pm 3.079$ GFAP-IR; amc $=53.030 \pm 14.415$ GFAP-IR; PD = $116.404 \pm 20.632$ GFAP-IR), although interindividual differences within one group were pronounced (Figure 3.1/2-B). The analysis of GFAP-IR in the SNpc (Figure 3.1/2-A, lower panel) did not show significant differences in PD patients $(P D=170.430 \pm 33.598$ GFAP-IR) compared to young ( $\mathrm{yc}=87.215 \pm 32.350$ GFAP-IR) or age-matched controls (amc $=143.005 \pm 38.409$ ) (Figure 3.1/2-C). However, there seemed to be a trend to enhanced astrogliosis in PD patients and age-matched controls. 

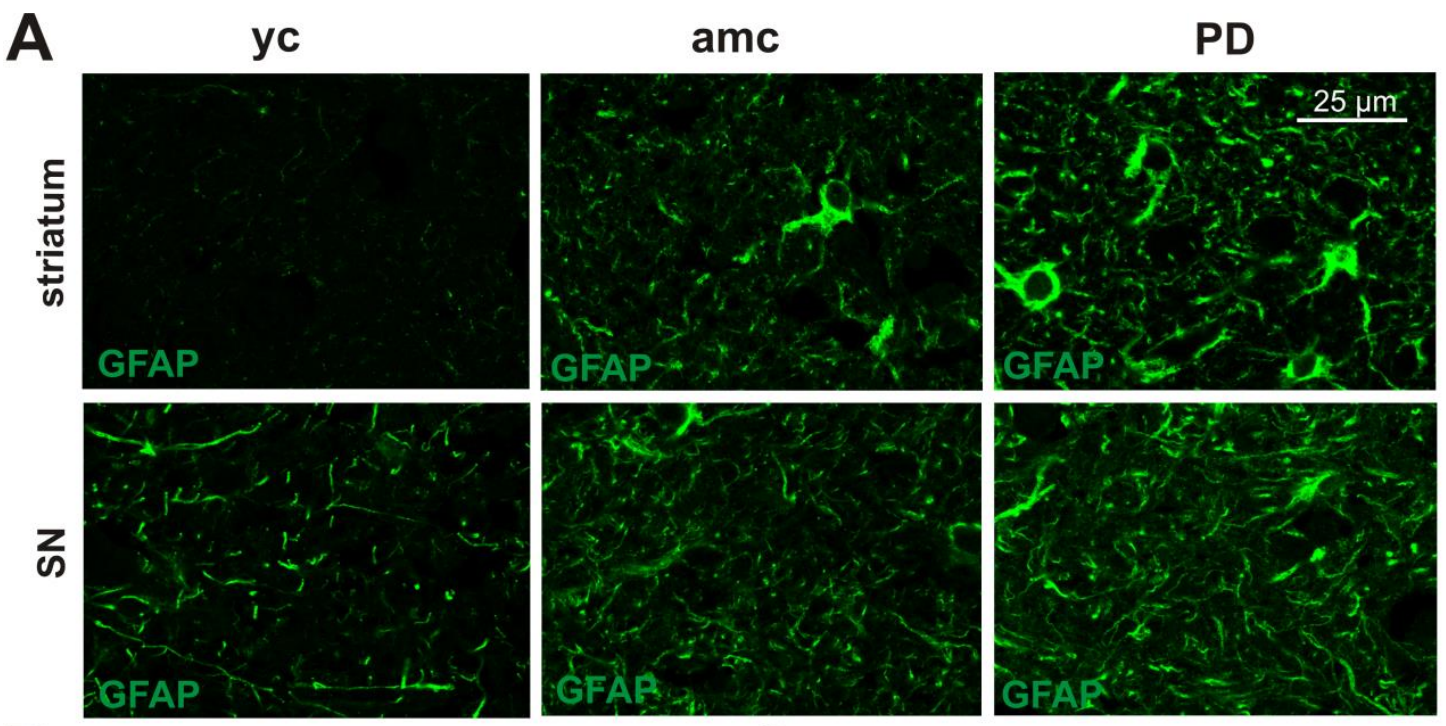

B

Astrocytes in striatum

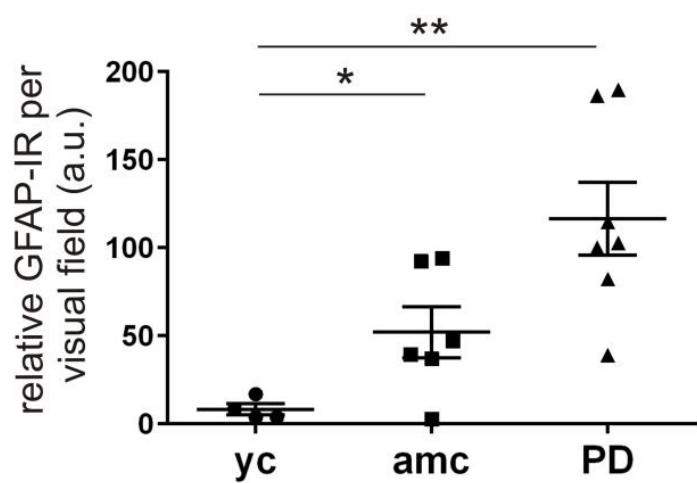

C Astrocytes in SNpc

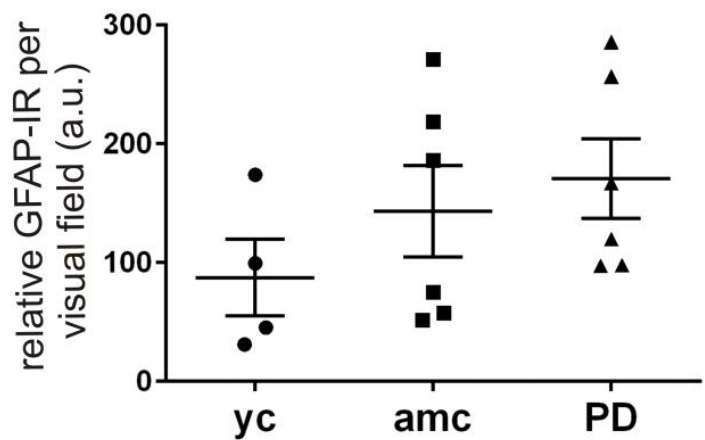

Figure 3.1/2: Immunohistochemical examination of astrocytes in the SNpc and striatum in human brain sections with the astrocytic marker GFAP. Representative micrographs from the putaminal striatum showed an increased GFAP-IR signal in PD cases compared to controls (A, upper panel, quantified in B: yc: $n=4$; amc: $n=6$; PD: $n=7$ ), while in the SNpc no difference in GFAP-IR was detectable ( $A$, lower panel, quantified in C: yc: $n=4$; amc: $n=6 ; P D: n=6)$. Data is given in averaged values from each case, bars represent means per group \pm SEM. ${ }^{*} \mathrm{P}<0.05 ;{ }^{*} \mathrm{P}<0.01$ (One-way ANOVA with Tukey Kramer test for independent comparison between groups).

To assess the inflammatory response to dopaminergic degeneration in the nigrostriatal system in PD an immunohistochemical analysis of microglia infiltration was performed. Immunolabeling of Iba1 denotes all microglia, thus the amount of Iba1-IR cells in the striatum was examined (Figure 3.1/3-A). The quantification of the Iba1 immunoreactivity (Iba1-IR) in the striatum resulted in no significant differences after group comparison (Figure $3.1 / 3-C$ ), possibly due to high interindividual variance in the young control group ( $y c=7.31$ $\pm 2.77 \mathrm{Iba1}-\mathrm{IR}$ ), which was not as pronounced in age-matched controls (amc $=2.93 \pm 0.9$ 
Iba1-IR). Interestingly, except one case, all PD brains showed less Iba1-IR (PD $=4.85 \pm 2.29$ Iba1-IR). The degeneration of dopaminergic neurons entailed the transformation of microglia into phagocytotic active microglia, which eliminate cell debris. In this state the phagocytic active microglia exhibit an amoeboid shape (Figure 3.1/3-B, arrows) and are considered to foster degeneration by producing detrimental molecules (Nakajima \& Kohsaka 2001). In order to identify phagocytic active microglia in human brains of PD cases and controls an immunolabeling for the macrophagic marker ED1 was performed (Figure 3.1/3B). Interestingly, there were no significant changes in the amount of ED1-IR cells observable, neither in the SNpc $(y c=10.25 \pm 0.680$ ED1-IR, $a m c=16.57 \pm 2.319$ ED1-IR, PD $=14.38$ \pm 2.114 ED1-IR cells per visual field, data not shown) nor in the striatum (yc $=7.32 \pm 0.591$, amc $=9.6 \pm 1.018, P D=10.9 \pm 1.220$ ED1-IR cells per visual field), however there were pronounced interindividual differences in the PD group (Figure 3.1/3-D). 

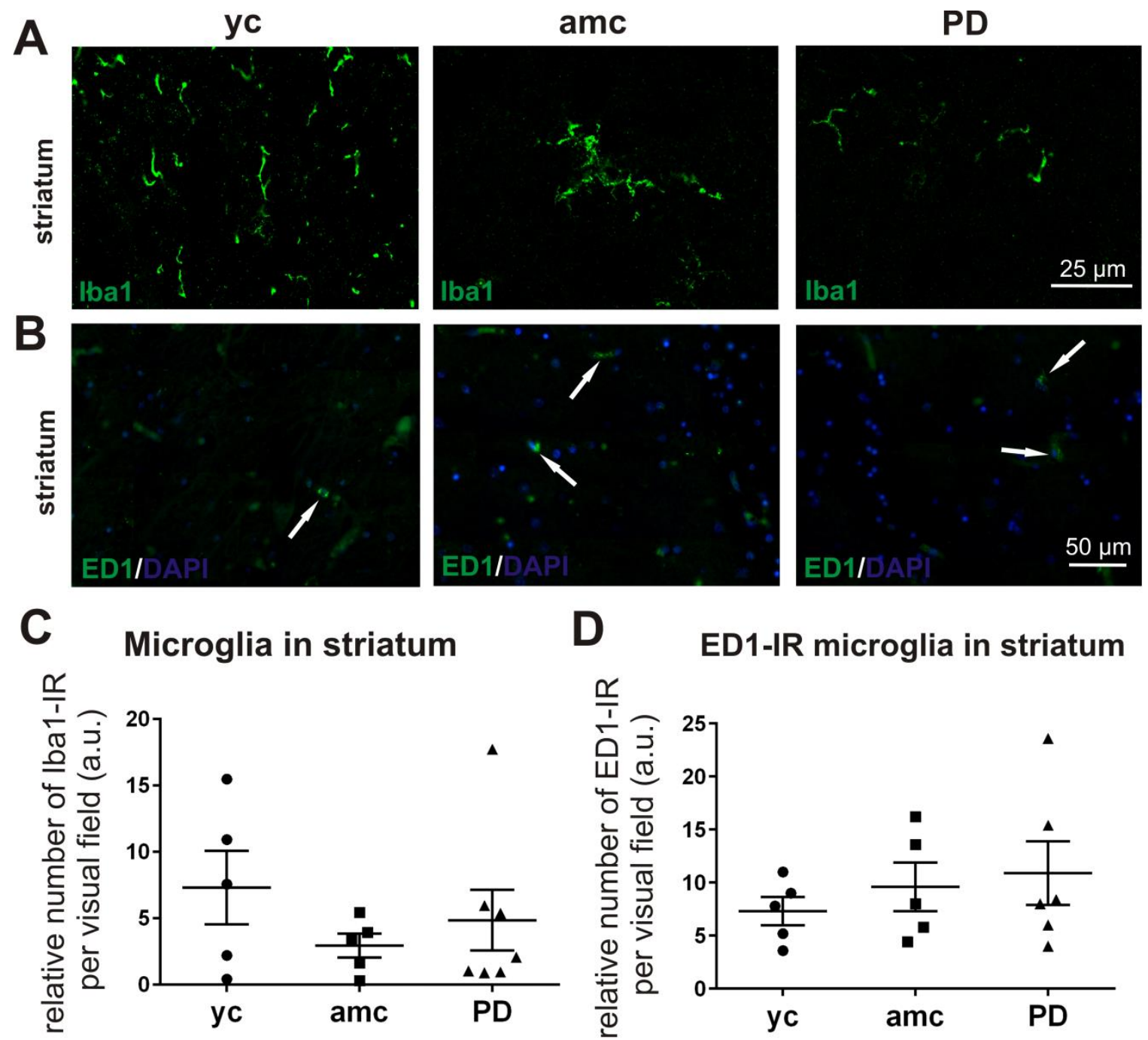

Figure 3.1/3: Immunohistochemical assessment of infiltrated microglia into the striatum on human brain sections. Staining the entire microglia in the putamen with lba1 is represented by micrographs of each group in A and the statistical quantification of Iba1-IR cells is displayed in B (yc: $n=5 ;$ amc: $n=5 ;$ PD: $n=7$ ) resulting in no differences in microglia numbers. Exemplary images of ED1-IR phagocytic microglia (white arrows) in the striatum are shown in C. Quantitative evaluation showed no differences in ED1-IR between the groups (D, yc: $n=5$; amc: $n=5 ; P D: n=6$ ). Data is given in averaged values from each case; bars represent means per group \pm SEM.

\subsubsection{Analysis of growth-associated proteins and presynaptic configuration}

To investigate the intrinsic regeneration capacity of the nigrostriatal system the main focus of the study included the investigation of the axonal growth-associated protein- 43 (GAP-43). To evaluate the expression levels of this protein, especially in dopaminergic neurons, co-staining of GAP-43 together was TH were performed. Interestingly, striatal TH 
fibers were rarely detectable, only very thick ones were labelled sporadically by the antibody. Thus co-localization of TH and GAP-43 was not possible in the striatum of human brain sections. Consequently GAP-43 expression was evaluated from the immunofluorescent signal in all tissue structures of the putamen. Surprisingly, the GAP-43-IR did not indicate any apparent differences in expression between the groups (Figure 3.1/4-A), thus the quantification resulted in no detectable changes in mean GAP-43 protein expression in PD cases $(\mathrm{PD}=47.141 \pm 3.023 \mathrm{GAP} 43-\mathrm{IR})$ compared to age-matched $(\mathrm{amc}=26.302 \pm 4.833$ GAP-43-IR) and young controls (yc = 27.141 \pm 2.756 GAP-43-IR) (Figure 3.1/4-B).

To assess the density of striatal pre-synaptic connections, the synaptic vesicle protein synaptophysin (synphy) was detected by immunohistochemical labeling. Similar to the GAP-43 expression, the density of the synphy fluorescence signal seemed to be not altered in amc or PD cases (Figure 3.1/4-C), however the quantification of the mean synphy-immunoreactivity (synphy-IR) showed a slight trend to decreased synaptophysin density in PD cases. The quantification showed no significant changes of the synaptic protein (yc $=10.849 \pm 1.526$ synphy-IR, amc $=11.738 \pm 1.011$ synphy-IR, PD $=8.469 \pm 2.402$ synphy$I R$, Figure 3.1/4-D), probably due to strong interindividual differences in the PD group.

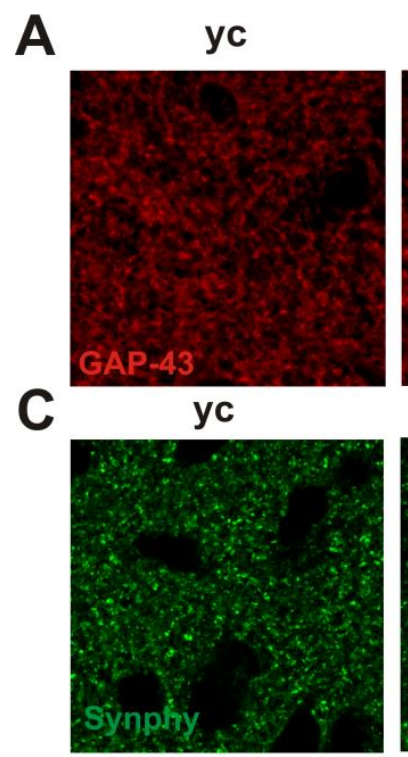

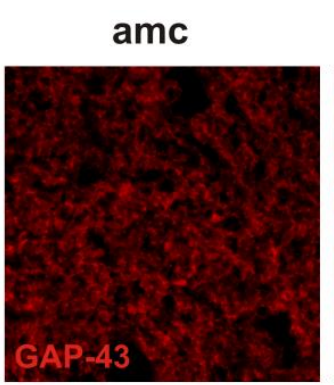

amc

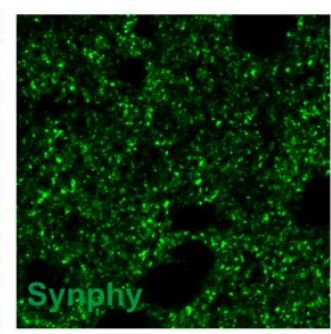

PD

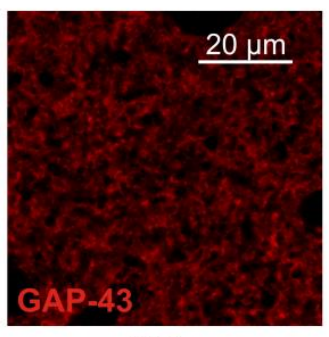

PD

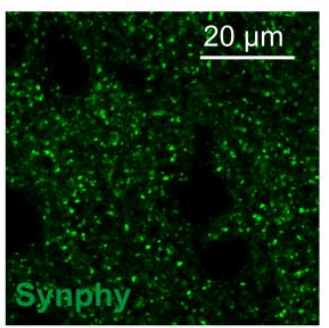

B

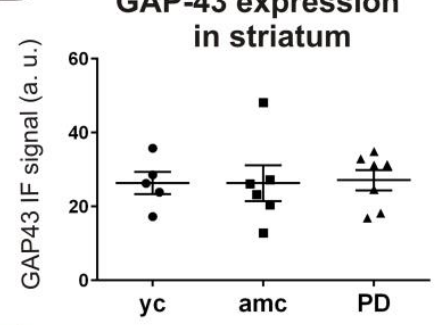

D Synaptophysin expression

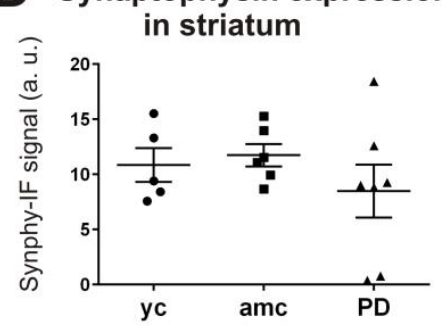

Figure 3.1/4: Analysis of striatal GAP-43 expression and synaptic integrity. Representative micrographs of GAP-43-immunofluorescence (IF) signal in the putamen of young controls (yc), age-matched controls (amc) and PD cases (PD) (A). Evaluation of GAP-43 protein expression in the striatum resulted in similar mean fluorescence signal in the groups (B, yc: $n=5 ;$ amc: $n=6 ;$ PD: $n=7$ ). The putaminal synaptophysin immunofluorescence (synphy-IF) is exemplary illustrated in $\mathbf{C}$, and the statistical group comparisons did not 
show obvious differences ( $D, y c: n=5$; amc: $n=6$; PD: $n=7)$. Data is given in averaged values from each case; bars represent means per group \pm SEM (One-way ANOVA).

In the SN the dopaminergic neurons were clearly identified by immunofluorescent staining against TH. To examine the GAP-43 protein amount in dopaminergic somata and processes, co-stainings for TH and GAP-43 were performed (Figure 3.1/5-A). One difficulty in measuring the GAP-43 amount in TH-IR cells was the strong presence of neuromelanin, thus the GAP-43 amount had to be quantified in areas of the cell where neither the nucleus nor neuromelanin was localized. This evaluation revealed sparse GAP-43-IR in dopaminergic cells and there were no differences in mean expression levels detectable between the groups (yc $=38.780 \pm 2.064, \mathrm{amc}=34.150 \pm 3.317, \mathrm{PD}=29.584 \pm 2.111$, Figure $3.1 / 5-\mathrm{B})$. There was $\mathrm{a}$ distinct trend for decreased GAP-43 protein expression in TH-IR cells in PD cases, which however was not statistically relevant. In contrast, the adjacent SNpc neuropil showed strong GAP-43-IR in all cases and a significantly higher mean GAP-43 protein expression in young controls ( $y c=65.571 \pm 4.801 \mathrm{GAP}-43-\mathrm{IR}$ ), while in age-matched controls and PD cases the GAP-43 signal was less intense $(\mathrm{amc}=43.965 \pm 2.953, \mathrm{PD}=42.29 \pm 4.271$ GAP43-IR, Figure $3.1 / 5-C)$. To allocate the GAP-43 expression to certain structures in the nigral neuropil, it was correlated to synaptophysin expression. Co-stainings of both proteins showed no significant co-localization, thus the GAP-43 expression primarily seems to be outside of the presynapse (Figure 3.1/5-A, last panel). In addition to the finding of decreased nigral GAP-43 protein expression in PD, the averaged synphy-IR was also intensely reduced in the SNpc (Figure 3.1/5-D) of PD cases (PD = $5.282 \pm 1.450$ synphy-IR), however interindividual synaptophysin expression was highly variable. In age-matched controls this decrease in synaptic configuration seems not as pronounced as in PD cases (amc $=7.048 \pm 1.140$ synphy-IR), nevertheless there is an apparent trend to diminished synphy-IR compared to young controls ( $y c=10.905 \pm 0.895$ synphy-IR). 
A
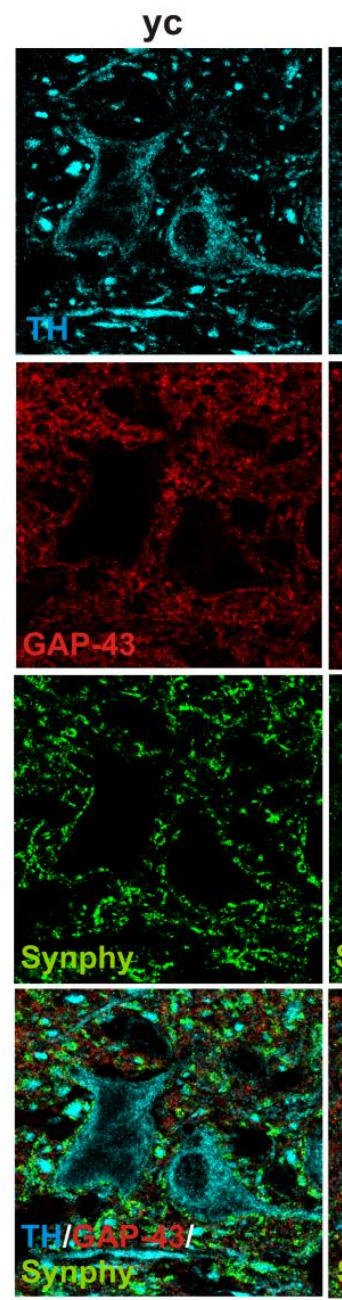
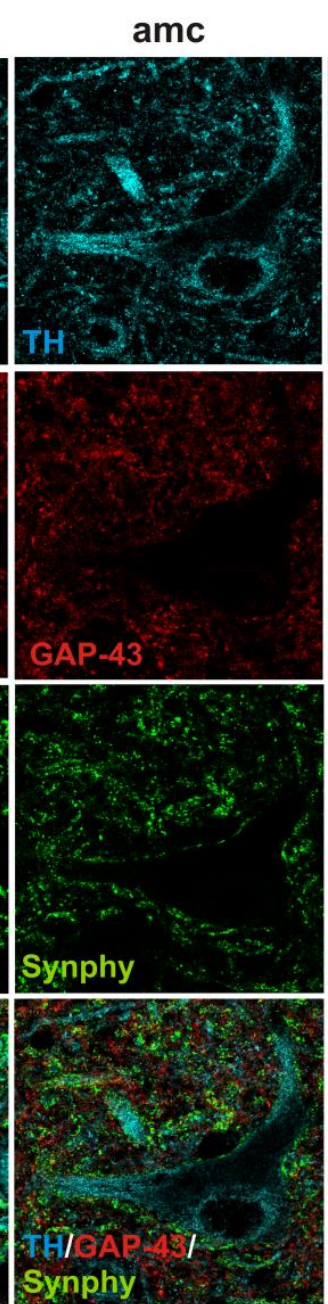
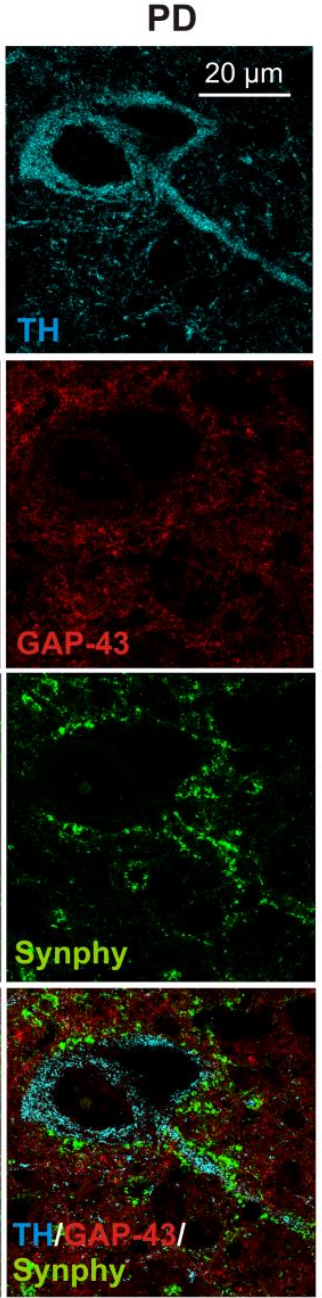

B GAP-43 expression in dopaminergic neurons

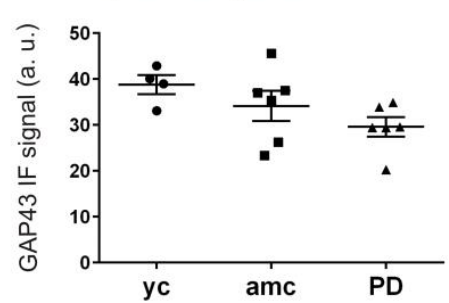

C GAP-43 expression in neuropil of SNpc

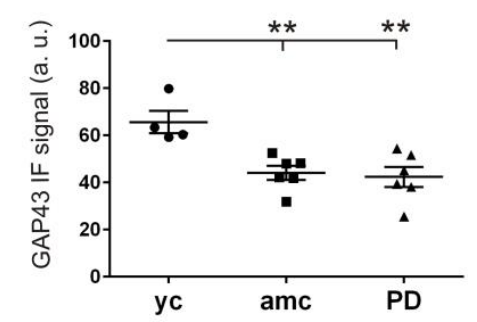

D Synaptophysin expression in neuropil of SNpc

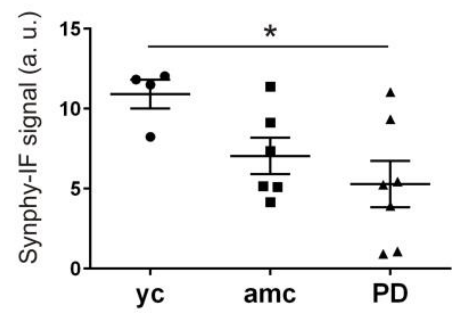

Figure 3.1/5: GAP-43 expression in dopaminergic neurons and neuropil of SN. Representative micrographs of multiple immunostainings of TH, GAP-43 and synaptophysin in the SNpc are illustrated in A. Results from the statistical evaluation of GAP-43-IR in the TH-IR cell somata (B) and in the nigral neuropil (C) depicted a decline in GAP-43 protein expression in the amc and PD groups compared to yc (B/C: yc: $n=4 ;$ amc: $n=6 ; P D: n=6)$. Synaptic composition is demonstrated by representative micrographs of synaptophysin protein expression in the SNpc (A) and the corresponding statistical analysis of synphy-IR is visualized in graph $D$ (yc: $n=4 ; a m c: n=6$; PD: $n=7)$. Data is given in averaged values from each case; bars represent means per group \pm SEM. ${ }^{*} P<0.05$; ${ }^{*} \mathrm{P}<0.01$ (One-way ANOVA with Tukey Kramer test for independent comparison between groups).

\subsubsection{Investigation of the growth-inhibitory protein Rho kinase (ROCK2) in}

\section{human brain sections}

Complementary to the pro-regenerative protein GAP-43, we were additionally interested in the role of the growth-inhibitory protein ROCK2 in PD. One characteristic feature of active ROCK is to induce, via phosphorylation of downstream proteins, stress fiber 
formation and growth cone collapse, leading to inhibition of regenerative fiber elongation. Immunohistochemical signals in the SNpc from PD cases and controls disclosed a very weak expression of this protein. Interestingly, ROCK2 showed a distinct puncta-like staining with ubiquitous distribution, less pronounced in TH-IR somata as in the neuropil (Figure 3.1/6-A). The evaluation of possible alterations in ROCK2 expression did not indicate any significant differences in expression between the groups, neither in the somata of dopaminergic neurons nor in the neuropil of the SNpc (Figure 3.1/6-B and 3.1/6-C), however with pronounced interindividual differences in protein expression in all groups.

A

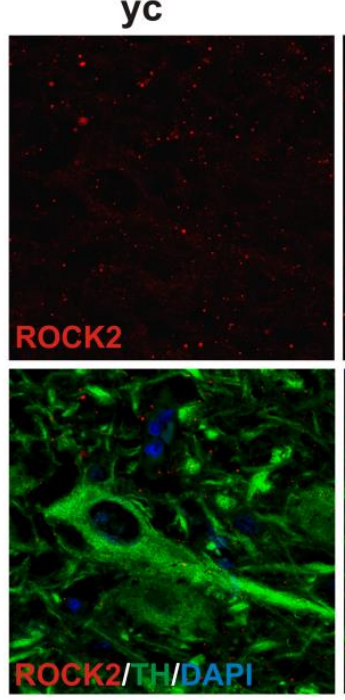

amc
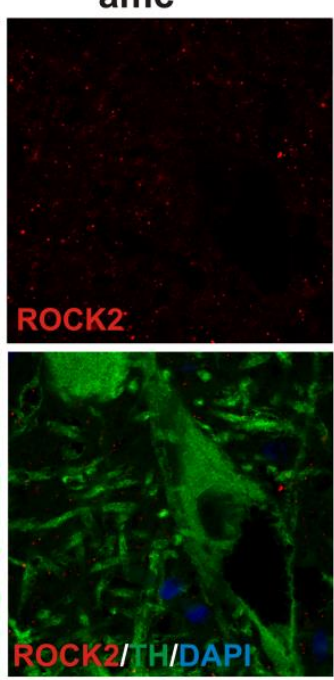

PD
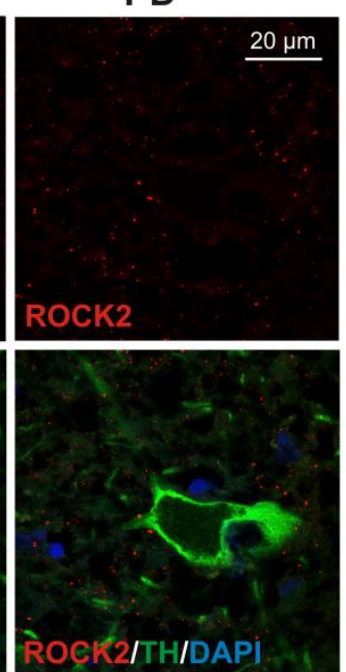

B ROCK2 expression in DAergic cells of the SNpc

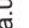

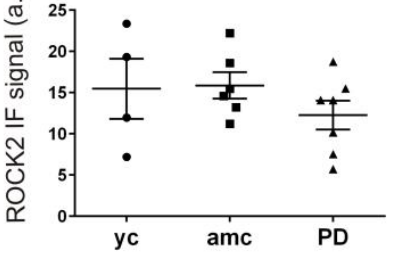

C ROCK2 expression in

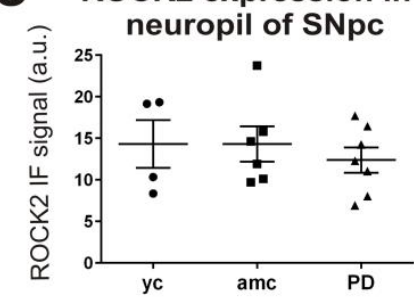

Figure 3.1/6: Analysis of ROCK2 expression in the substantia nigra of PD cases and controls. Representative micrographs of ROCK2-IR are illustrated in A, upper panel and double immunoreactive ROCK2/TH co-stainings in A, lower panel. ROCK2-IR was evaluated in TH-IR cells and quantified in B. ROCK-IR in the adjacent neuropil was measured in TH-negative areas and the quantification is illustrated in $\mathbf{C}$. The statistical comparison resulted in no differences in expression levels between the groups (B/C, yc: $n=4 ;$ amc: $n=6 ; P D: n=7)$. Data is given in averaged values from each case; bars represent means per group \pm SEM (One-way ANOVA).

In the striatum, the ROCK2 protein expression showed similar distribution patterns of the punctate structures as in the SNpc. The overall ROCK2-IR signal was quite low in all sections (Figure 3.1/7-A). Measuring the amount of averaged ROCK2-immunofluorescence (ROCK2-IF) in the entire striatal tissue structures, by randomly chosen areas from the acquired images, resulted in no clear expression changes in the groups (Figure 3.1/7-B). Interestingly, in PD cases and age-matched controls, a number of single cells with an 
intensive ROCK2-immunoreactivity (Figure 3.1/7-A, arrows) could be noticed, which were quantified in number. We discovered a distinct increase in these ROCK2-IR cells in PD cases (PD $=34.013 \pm 5.288$ ROCK-IR cells/visual field) as compared to young controls (yc = $17.177 \pm 3.0$ ROCK-IR cells/visual field). A similar increase was observed in age-matched controls as well (amc $=32.342 \pm 3.471$ ROCK-IR cells/visual field, Figure 3.1/7-C), but this was not significantly different to young controls. 

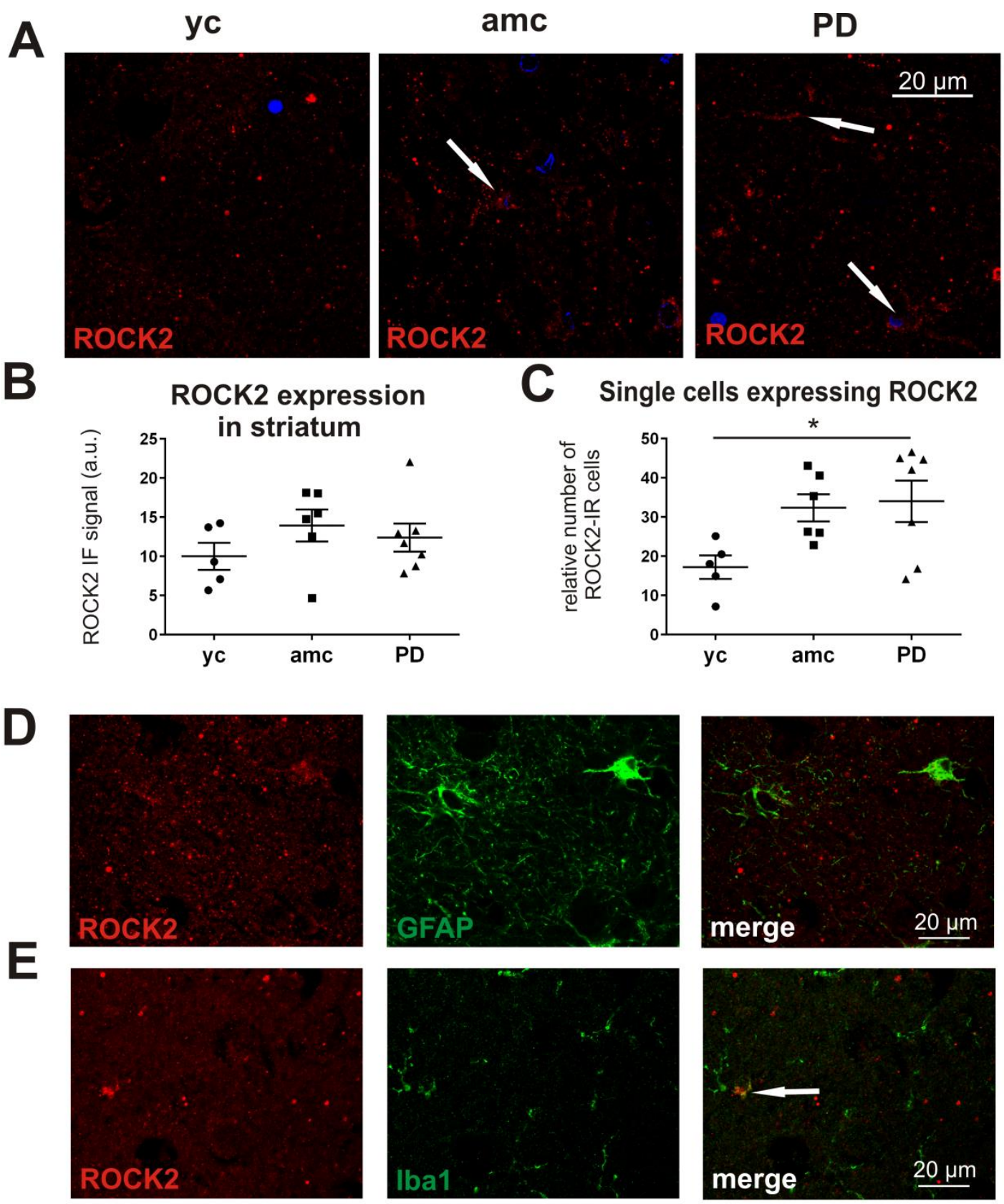

C Single cells expressing ROCK2

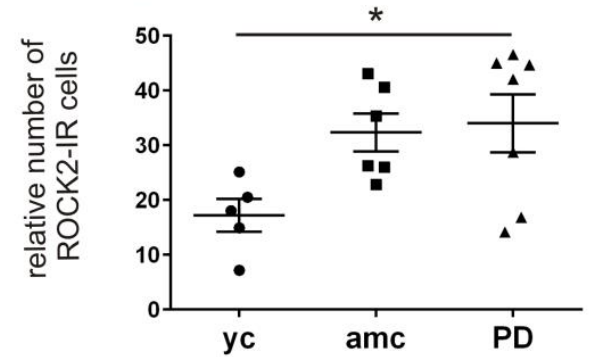

E

ROCK2-IR astroytes
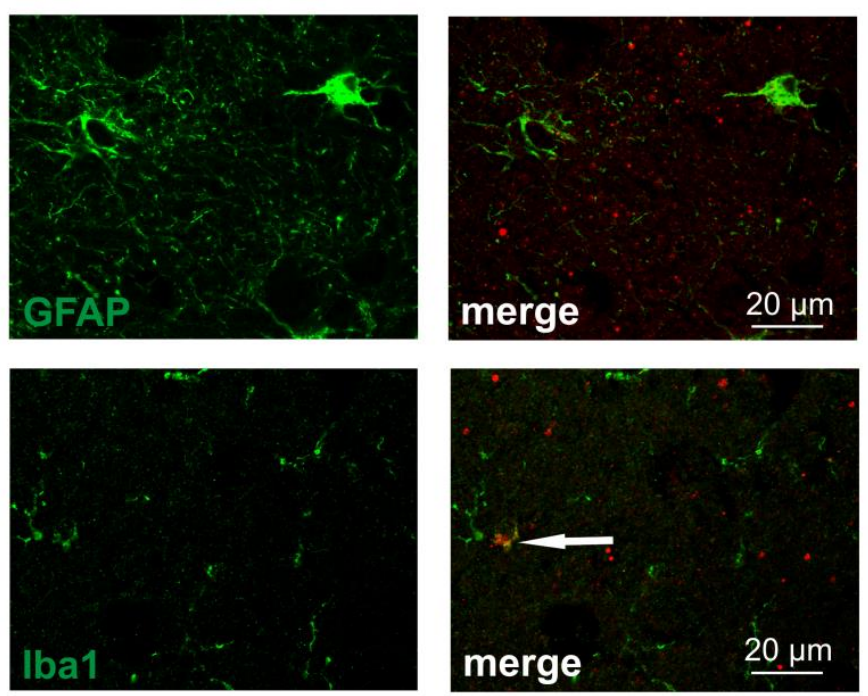

$F$
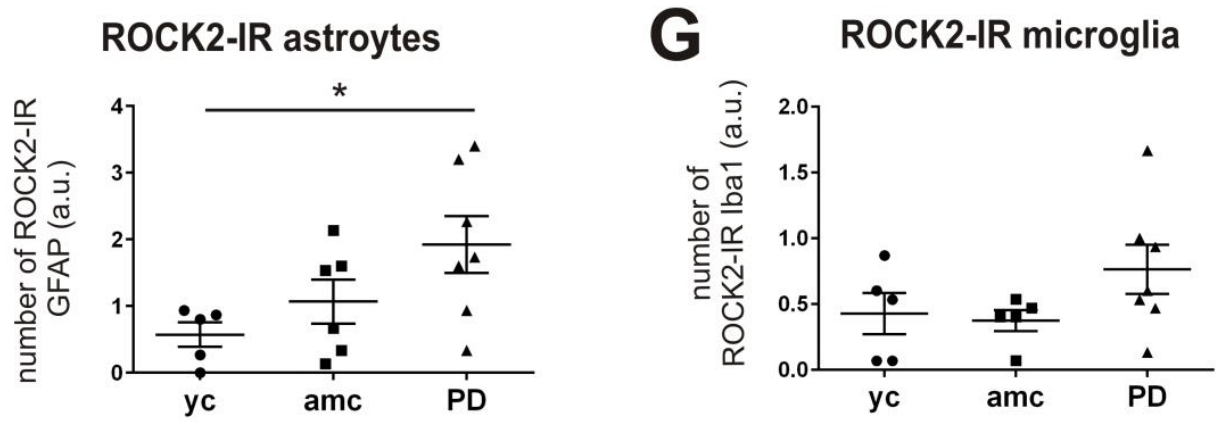

Figure 3.1/7: Quantification of ROCK2-IR in the striatum of PD cases and controls. Representative micrographs illustrate the ROCK2 protein expression in the putamen of young and age-matched controls and PD cases (A). The evaluation of the general ROCK2 fluorescence signal resulted in no detectable differences between the groups (B, yc: $n=4$; amc: $n=6$; PD: $n=7$ ). White arrows point to single cells with high ROCK-IR (A). Quantification 
of these ROCK-IR cells resulted in significantly increased numbers in PD cases (C, yc: $n=5 ;$ amc: $n=6 ;$ PD: $n=7$ ). These cells could be identified by double-immunostaining of ROCK2 and GFAP as astrocytes (D) and with costaining of ROCK2 and Iba1 partially as microglia (E). Quantification of these double-immunoreactive cells resulted in significantly increased numbers of ROCK-IR astroglia ( $F, y c: n=5$; amc: $n=6$; PD: $n=7$ ) and in slightly enhanced counts of ROCK2-IR microglia (G, yc: $n=5$; amc: $n=5 ; P D: n=7)$. Data is given in averaged values from each case, bars represent means per group \pm SEM. ${ }^{*} \mathrm{P}<0.05$ (One-way ANOVA with Tukey Kramer test for independent comparison between groups).

In order to clarify the role of these ROCK2-IR cells during ageing or in the development of PD, we tried to identify these cells. Because of their morphology these cells appeared potentially as glial cells, featuring processes and smaller nuclei than neurons. With immunostainings against GFAP and Iba1 in combination with ROCK2 (Figure 3.1/7-D and E) the amount of ROCK2-IR astrocytes and microglia was quantified. The analysis revealed that the average number of ROCK-IR astrocytes was significantly higher in PD cases (PD $=1.924 \pm 0.425$ ROCK2/GFAP-IR cells/visual field) compared to young (yc $=0.573 \pm 0.186$ ROCK2/GFAP-IR cells/visual field) and age-matched controls (amc $=1.067 \pm 0.327$ ROCK2/GFAP-IR cells/visual field). However, also age-matched controls tended to show increased numbers of ROCK2-IR astrocytes (Figure 3.1/7-F), including pronounced interindividual differences. Regarding the double staining of Iba1 and ROCK2 the analysis revealed no striking co-labeling of microglia in any groups (Figure 3.1/7-E). In the PD group there were three cases with remarkable amount of ROCK-IR microglia, however the mean number of ROCK-IR and Iba1-IR cells was not significantly increased in PD cases (PD $=0.76 \pm 0.2$ ROCK2/Iba1-IR cells/visual field) compared to the controls $(\mathrm{yc}=0.43 \pm 0.16 \mathrm{ROCK} 2 / \mathrm{Iba1}-\mathrm{IR}$ cells/visual field and amc $=$ $0.37 \pm 0.08$ ROCK2/Iba1-IR cells/visual field) (Figure 3.1.7-G). Taken together, there was evidence for an elevated number of striatal astrocytes that express ROCK2 in conspicuous amounts, which was accompanied with a trend to more ROCK2-IR microglia in PD cases. These findings would explain the higher number of striatal ROCK-IR cells in brains from PD cases, suggesting an important role forROCK2 in striatal glial cells in the progression of PD. 


\subsection{The role of ROCK2 inhibition in the 6-OHDA mouse model of PD}

Since it is known that pharmacological ROCK2 inhibition can directly or indirectly affect the neuronal survival and regeneration, we examined in the present investigation the neuron-specific impact of virus-mediated ROCK2 downregulation in the 6-OHDA mouse model of PD. To determine the neuroprotective potential of AAV.shRNA-mediated inhibition of ROCK2, mice received a unilateral right-sided striatal 6-OHDA lesion targeting the axonal terminals of the nigrostriatal tract. Simultaneously they were injected with a neuron specific AAV.ROCK2-shRNA into the ipsilateral SN with two different virus titers.

\subsubsection{AAV mediated gene transfer leads to effective dsRed expression in neurons of the substantia nigra}

In order to confirm the successful transduction of the shRNA construct into neurons of the SNpc and to validate the neuronal specificity of the AAV2, a co-localization of the vector-expressed fluorophore dsRed and different marker proteins for neurons and glial cells was performed. The cell type driven fluorophore expression was exclusively co-localized with the immunohistochemical staining against the neuronal nuclei marker NeuN for the viral vector carrying the ROCK2-shRNA as well as the respective control shRNA (Figure 3.2/1-A). Immunostainings for the astrocytic marker GFAP and the microglia epitope Iba1 resulted in no detectable co-localization with the vector-driven dsRed fluorophore (Figure 3.2/1-B and C). Thus, co-localization of the NeuN-immunoreactivity (NeuN-IR) fluorescence signal and dsRed revealed strong evidence for an effective transduction of neurons by ROCK2-shRNA. 
3. Results
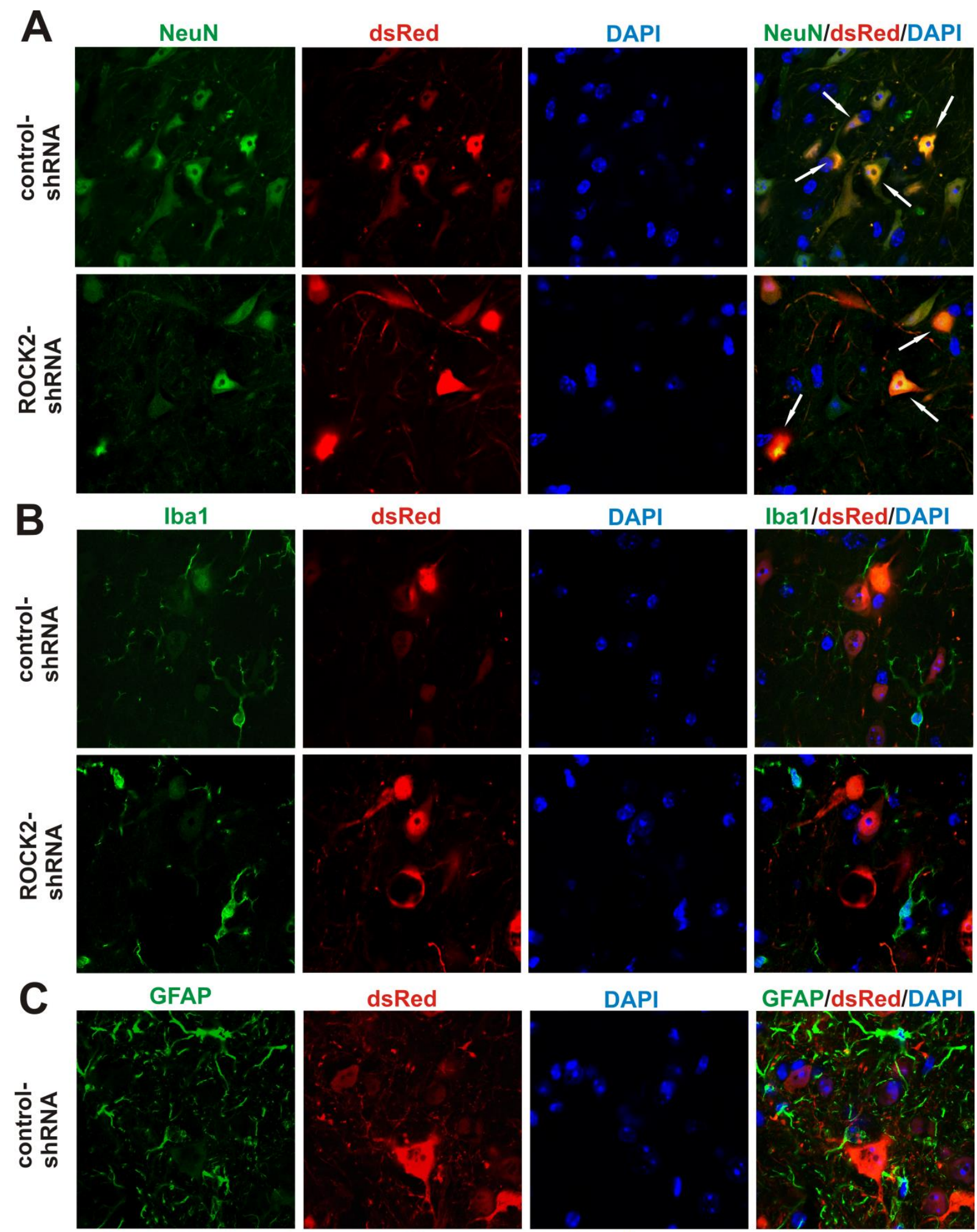

GFAP/dsRed/DAPI
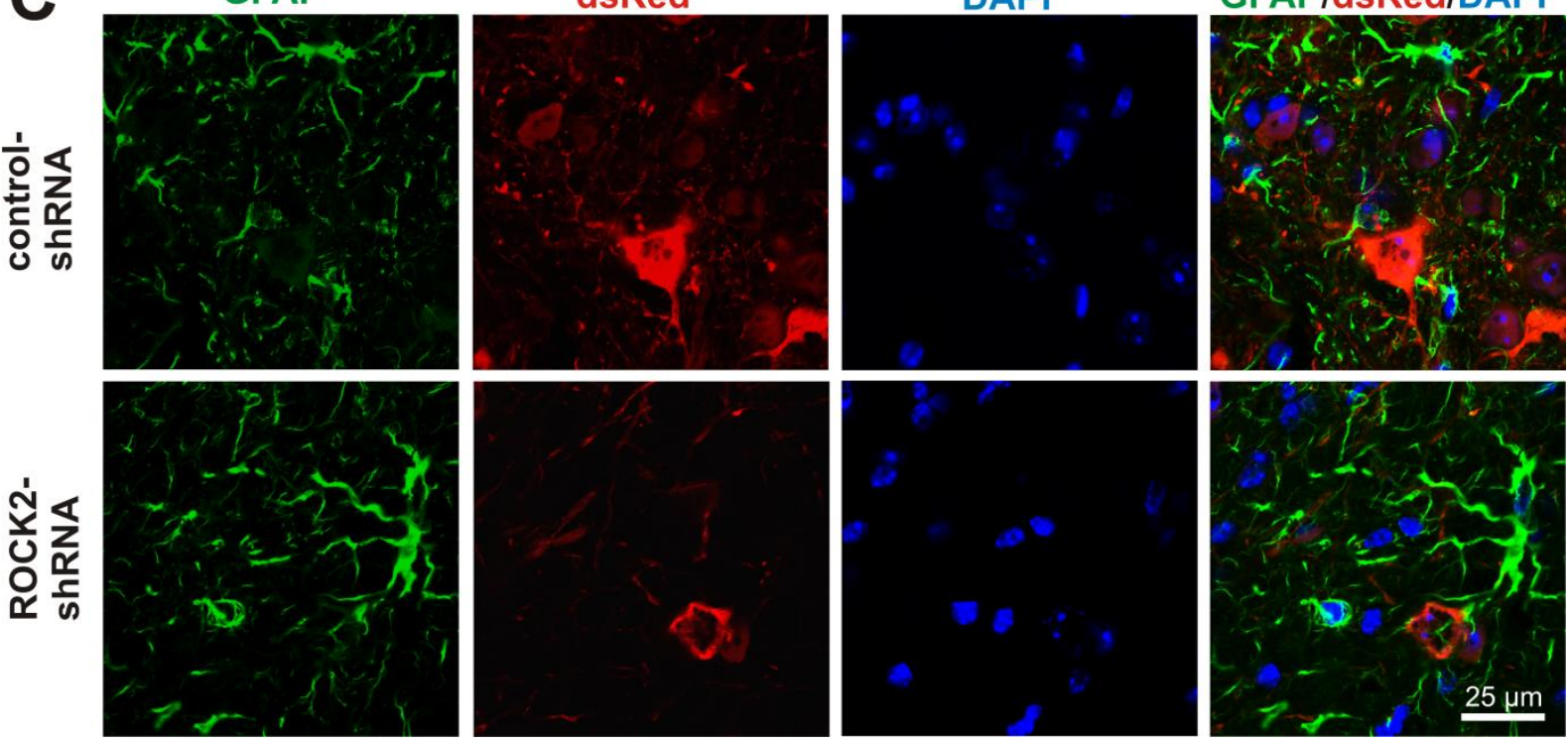
Figure 3.2/1: Immunohistological demonstration of AAV transduction into neurons of the substantia nigra in vivo. The co-localisation of the intracellularly expressed fluorophore dsRed and the neuronal marker NeuN showed evidence for targeting nigral neurons with either the control- or the ROCK2-shRNA (A, arrows depict co-localisation of NeuN and dsRed). The cell type specificity of the synapsin-promoter-driven dsRed expression is underscored by additional immunolabelings for Iba1 (B) and GFAP (C).

\subsection{2. shRNA-mediated ROCK2 downregulation increases dopaminergic neuron survival after 6-0HDA intoxication}

At four weeks after the lesion the immunohistochemistry of TH-IR structures demonstrated a drastic loss of TH-IR cells in the ipsilateral SNpc (Figure 3.2/2) caused by the unilateral 6-OHDA intoxication. The quantification of TH-IR cells of the ipsilateral SN via stereological counting displayed a significant decrease of about $60 \%$ of TH-IR neurons in 6-OHDA lesioned animals in comparison to vehicle controls (LAA/PBS $=11083.2 \pm 503.4$ cells vs. 6-OHDA/PBS $=4019.2 \pm 640.2$ cells, Figure 3.2/2-A-B). Simultaneous treatment with ROCK2-shRNA in animals lesioned with 6-OHDA resulted in an attenuated loss of TH-positive neurons (Figure 3.2/2D-F). In detail, animals transduced with AAV.ROCK2-shRNA in a titer of $0.25 \times 10^{8} \mathrm{TU}$ had significantly more TH-positive neurons as compared to control-shRNA (6-OHDA/ROCK2-shRNA0.25 $=6529.2 \pm 696.9$ cells vs. $6-$ OHDA/control-shRNA $=$ $2982.1 \pm 704.3$ cells; Figure 3.2/2-F). Introduction of the higher titer $\left(1.0 \times 10^{8} \mathrm{TU}\right)$ of AAV.ROCK2-shRNA also resulted in an increased cell survival after 6-OHDA intoxication, however not reaching statistical significance as compared to control virus (6-OHDA/ROCK2shRNA1.0 = $4982.9 \pm 645.1$ cells; Figure 3.2/2-F). 


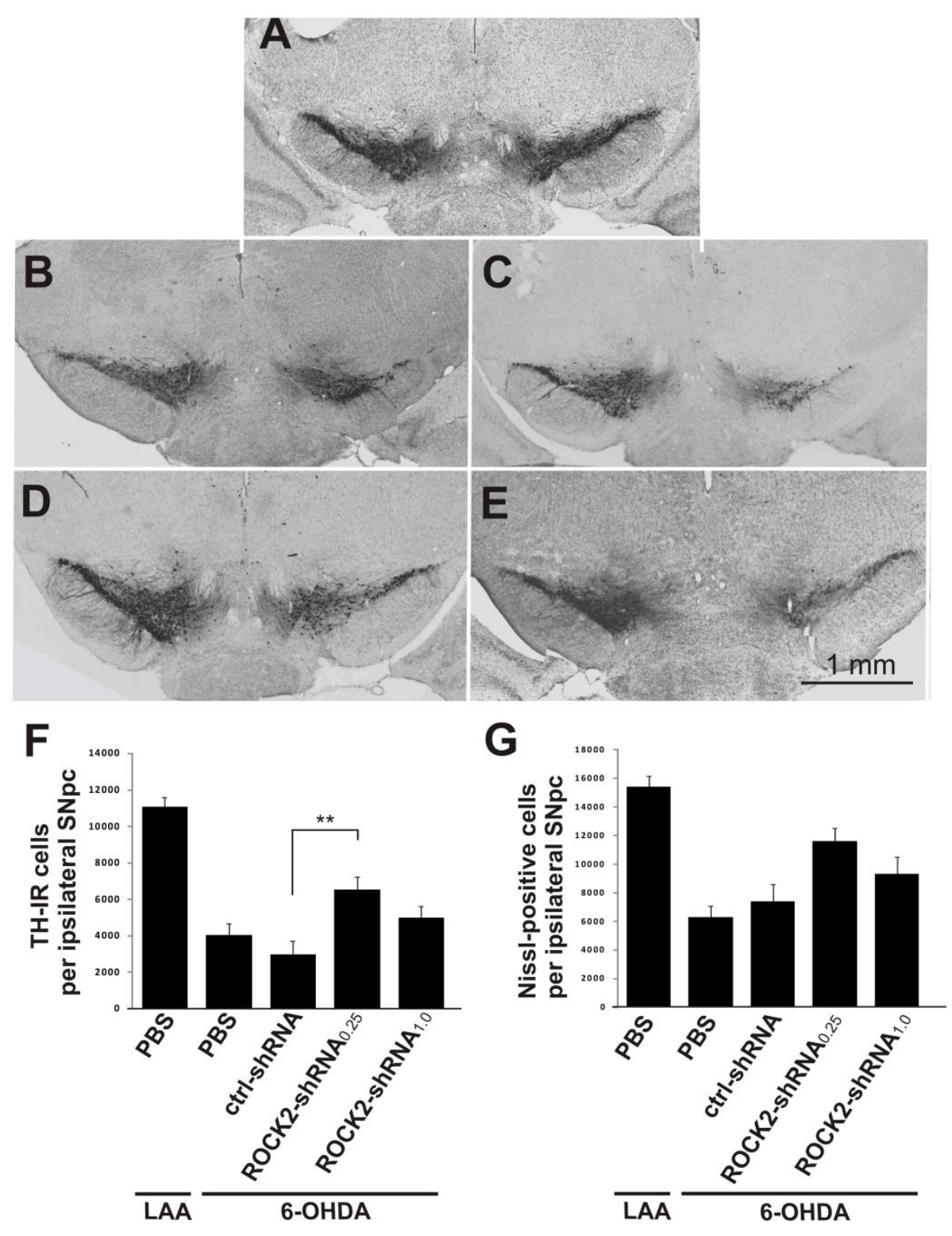

Figure 3.2/2: Immunohistological analysis of tyrosine hydroxylase (TH) and Nissl cells in the substantia nigra (SN) four weeks after 6-OHDA lesion of the striatum and introduction of a ROCK2-shRNA via viral vector into the SN. (A-E) Representative micrographs of SN immunolabeled against TH and treated with vehicle (LAA/PBS, A, $n=4), 6-O H D A / P B S ~(B, n=7), 6-O H D A / c o n t r o l-s h R N A$ in a titer of $1.0 \times 10^{8} \mathrm{TU} / \mu \mathrm{l}$ (ctrl-shRNA, C, $n=5$ ), 6-OHDA/ROCK2-shRNA in a titer of $0.25 \times 10^{8} \mathrm{TU} / \mu \mathrm{l}$ (ROCK2-shRNA0.25, D, n=4) or 6-OHDA/ROCK2-shRNA in a titer of $1.0 \times 10^{8} \mathrm{TU} / \mu \mathrm{l}$ (ROCK2-shRNA1.0, $\mathrm{E}, \mathrm{n}=5$ ). Quantitative stereological analysis of TH (F) and Nissl positive cells $(\mathbf{G})$ in the SNpc depicted a massive loss of TH-IR cells after 6-OHDA intoxication. Data are given as means \pm SEM. ${ }^{* * P}<0.01$ (one-way ANOVA with Dunnett's test compared to control-shRNA).

Nissl staining was performed to identify the entirety of all neurons in the SN. From the Nissl positive cell number it can be concluded whether the loss of TH-IR resulted from 
death of dopaminergic neurons or if there is a 6-OHDA-induced downregulation of $\mathrm{TH}$ expression and therefore cells would appear as Nissl positive anyway. As demonstrated in Figure 3.2/2-G, nigral 6-OHDA injection leads to a severe loss of Nissl positive cells in the ipsilateral SN, indicating robust dopaminergic degeneration in all of the lesioned groups $(\mathrm{LAA} / \mathrm{PBS}=15377.5 \pm 775.4$ cells vs. $6-\mathrm{OHDA} / \mathrm{PBS}=6301.8 \pm 753.9$ cells; $6-\mathrm{OHDA} /$ ROCK2shRNA0.25 $=11597.6 \pm 915.5$ cells and 6-OHDA/ROCK2-shRNA1.0 $=9312.7 \pm 1189.4$ cells vs. 6-OHDA/control-shRNA $=7407.2 \pm 1169.1$ cells; Figure 3.2/2-G). ROCK2-shRNA-treated groups showed the same trend of attenuated cell loss as depicted in TH-cell counting, confirming the neuroprotective effects on dopaminergic neurons.

\subsubsection{ROCK2 downregulation has only mild effects on dopaminergic fiber density and dopamine levels in the striatum}

To determine the protective effect of AAV.shRNA-mediated downregulation of ROCK2 on the nigrostriatal terminals after 6-OHDA lesion, the density of TH-positive fibers in the striatum was investigated. The relative striatal dopaminergic fiber density was examined by measuring the mean TH-immunoreactivity (TH-IR) from the lesioned as well as from the unlesioned hemisphere (TH-IR lesioned/TH-IR unlesioned). In the micrographs in Figure 3.2/3-B-E there is a severe decrease of TH-IR detectable in the dorsal striatum of intoxicated animals at four weeks after cerebral 6-OHDA injections. The quantification of TH-IR showed a significant reduction of approximately 55\% in the 6-OHDA control group as compared to vehicle injected animals $(\mathrm{LAA} / \mathrm{PBS}=98.5 \pm 1.2 \% \mathrm{TH}-\mathrm{IR}$ vs. $6-\mathrm{OHDA} / \mathrm{PBS}=44.1 \pm 5.3 \% \mathrm{TH}-\mathrm{IR}$; Figure 3.2/3). Animals simultaneously treated with AAV.ROCK2-shRNA in both titers displayed less fiber decline than the lesion group compared to the vehicle injected mice. However, there was only a strong trend towards attenuated TH-fiber loss in ROCK2 shRNAtreated animals by comparing them to the adequate control-shRNA group, not reaching significance due to the big variances in the groups (6-OHDA/ROCK2-shRNA0.25m $=72.2 \pm$ 6.2\% TH-fiber density and 6-OHDA/ROCK2-shRNA1.0 $=69.0 \pm 9.9 \% \mathrm{TH}$-fiber density vs. 6-OHDA/control-shRNA $=49.9 \pm 2.1 \% \mathrm{TH}$-fiber density; Figure 3.2/3-F) 


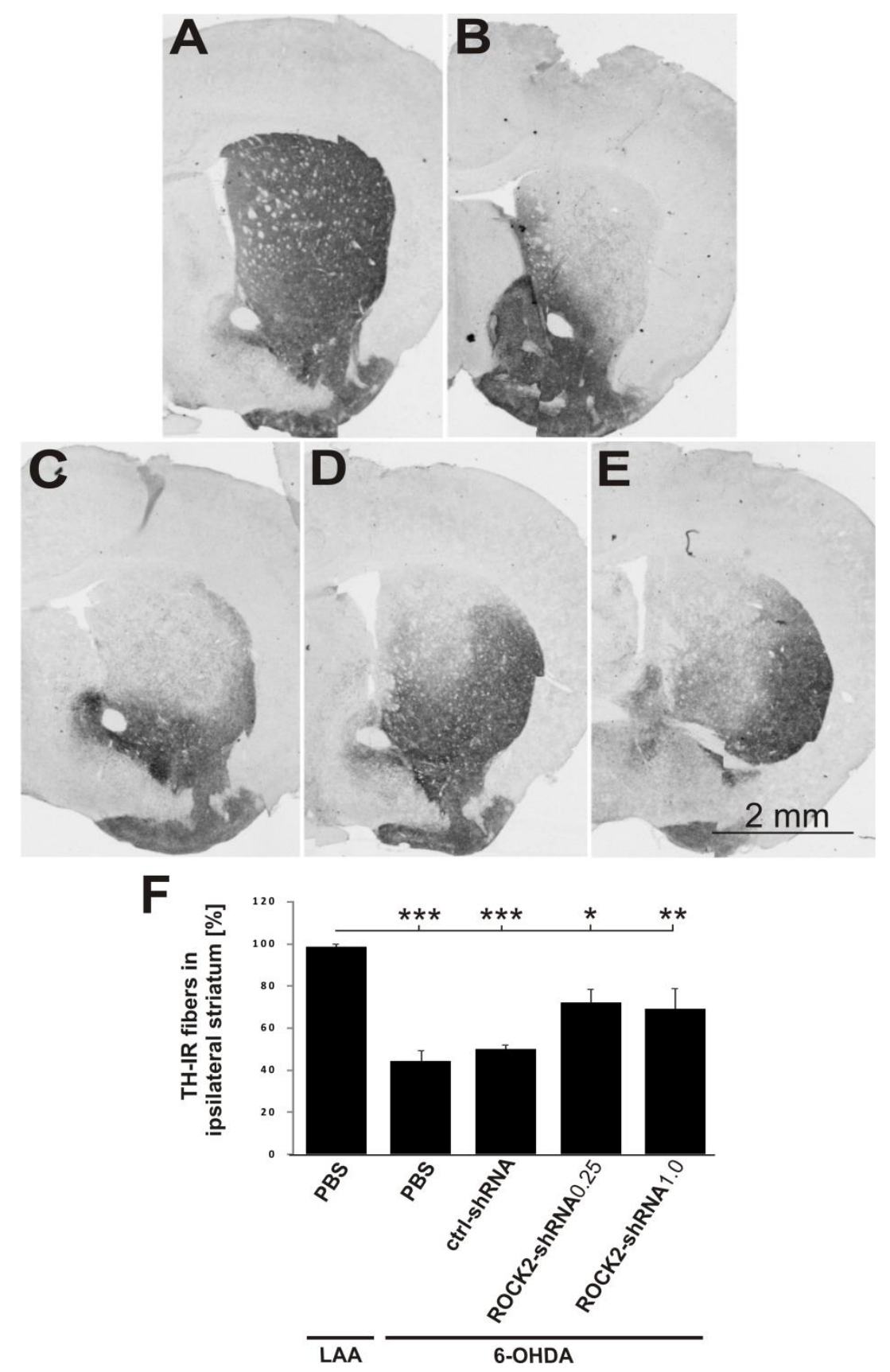

Figure 3.2/3: Immunohistological analysis of striatal TH density four weeks after 6-OHDA lesion of the striatum and introduction of a ROCK2-shRNA via viral vector into the SN. (A-E) Representative micrographs of injected striata immunolabeled against TH with the following groups: vehicle (LAA/PBS A, $n=5$ ), 6-OHDA/PBS (B, $n=7$ ), 6-OHDA/control-shRNA (ctrl-shRNA, C, n=5), 6-OHDA/ROCK2-shRNA in a titer of 0.25x 10 ${ }^{8}$ TU/ $\mu$ l (ROCK2shRNA0.25, D, $\mathrm{n}=5$ ) or 6-OHDA/ROCK2-shRNA in a titer of $1.0 \times 10^{8} \mathrm{TU} / \mu \mathrm{l}$ (ROCK2-shRNA1.0, $\mathrm{E}, \mathrm{n}=4$ ). Quantitative analysis of relative striatal TH fiber density (lesioned side/unlesioned side in \%) is depicted in F. Data is shown as means $\pm \mathrm{SEM}$. ${ }^{*} \mathrm{P}<0.05 ;{ }^{*} \mathrm{P}<0.01 ;{ }^{*} * \mathrm{P}<0.001$ (One-way ANOVA with Dunnett's test for comparison with vehicle group). 
In order to correlate the histological results of the striatal $\mathrm{TH}$-fiber density to a functional preservation of these axonal terminals a high performance liquid chromatography (HPLC) was performed four weeks after AAV.ROCK2-shRNA treatment. For this neurochemical analysis of dopamine and its metabolites fresh brain tissue was processed to purify the molecules for HPLC. Results from chromatographic detection depicted that the acute 6-OHDA intoxication had almost completely depleted the striatal levels of dopamine after 4 weeks (DA: LAA/PBS $=12.18 \pm 0.57 \mathrm{ng} / \mathrm{mg}$ vs. $6-\mathrm{OHDA} / \mathrm{PBS}=0.40 \pm 0.17 \mathrm{ng} / \mathrm{mg}$; Figure 3.2/4-A). This depletion of dopamine was consistent in all treatment groups and no effect of ROCK2-shRNA transduction could be observed (6-OHDA/ROCK2-shRNA0.25 = $0.51 \pm 0.18 \mathrm{ng} / \mathrm{mg}$ and 6-OHDA/ROCK2-shRNA1.0 = $2.07 \pm 1.01 \mathrm{ng} / \mathrm{mg}$ vs. 6-OHDA/control$\operatorname{shRNA}=1.44 \pm 0.56 \mathrm{ng} / \mathrm{mg})$.

Regarding to the dopamine metabolites HVA and DOPAC a similar depletion of these molecules was observed after 4 weeks, again with no detectable impact on metabolic levels between ROCK2-shRNA treated groups and controls (Figure 3.2/4-B: HVA: LAA/PBS = 1.98 $\pm 0.12 \mathrm{ng} / \mathrm{mg}$ vs. $6-\mathrm{OHDA} / \mathrm{PBS}=0.71 \pm 0.11 \mathrm{ng} / \mathrm{mg} ; 6-\mathrm{OHDA} / \mathrm{ROCK} 2-\operatorname{shRNA0} 0.25=0.8 \pm 0.06$ $\mathrm{ng} / \mathrm{mg}$ and 6-OHDA/ROCK2-shRNA1.0 $=0.83 \pm 0.18 \mathrm{ng} / \mathrm{mg}$ vs. $6-\mathrm{OHDA} /$ control-shRNA $=$ $0.97 \pm 0.12 \mathrm{ng} / \mathrm{mg}$ and Figure 3.2/4-C: DOPAC: LAA/PBS $=2.74 \pm 0.16 \mathrm{ng} / \mathrm{mg}$ vs. 6-OHDA/PBS $=0.26 \pm 0.1 \mathrm{ng} / \mathrm{mg} ; 6-\mathrm{OHDA} /$ ROCK2-shRNA0.25 $=0.3 \pm 0.12 \mathrm{ng} / \mathrm{mg}$ and 6-OHDA/ROCK2shRNA1.0 = $0.7 \pm 0.3 \mathrm{ng} / \mathrm{mg}$ vs. $6-\mathrm{OHDA} /$ control-shRNA $=0.78 \pm 0.27 \mathrm{ng} / \mathrm{mg}$ ).

A

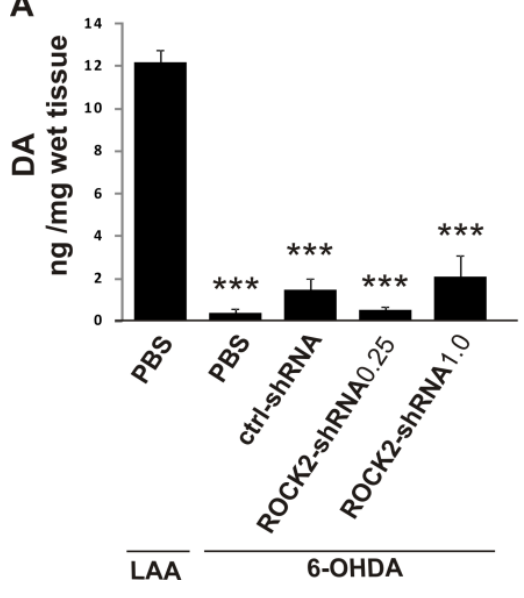

B

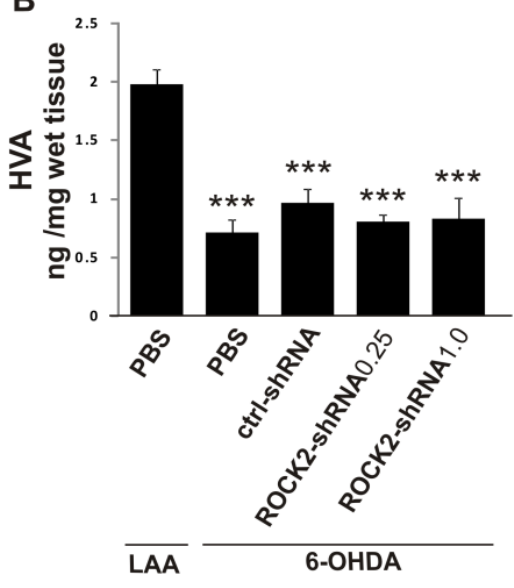

C

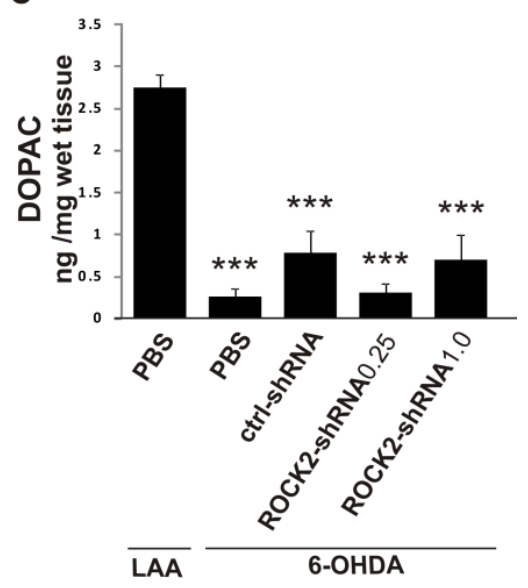

Figure 3.2/4: HPLC analysis of dopamine and dopamine metabolites in the striatum at four weeks after striatal 6-OHDA lesion and nigral transduction with AAV.ROCK2-shRNA. Quantification of dopamine (DA) (A), homovanillic acid (HVA) (B) and 3,4-dihydroxyphenylacetic acid (DOPAC) (C) resulted in no observable 
differences between the groups, included vehicle (LAA/PBS, $n=10), 6-O H D A / P B S(n=8), 6-O H D A / c o n t r o l-s h R N A$ (ctrl-shRNA, $\mathrm{n}=10$ ); 6-OHDA/ROCK2-shRNA in a titer of $0.25 \times 10^{8} \mathrm{TU} / \mu \mathrm{l}$ (ROCK2-shRNA0.25, $\mathrm{n}=10$ ) and 6-OHDA/ROCK2-shRNA in a titer of $1.0 \times 10^{8} \mathrm{TU} / \mu \mathrm{l}$ (ROCK2-shRNA1.0, $\mathrm{n}=11$ ). Data are given as means $\pm \mathrm{SEM}$. $* * * \mathrm{P}<0.001$ (One-way ANOVA with Dunnett's test compared to vehicle group).

\subsubsection{ROCK2 downregulation via AAV-shRNA has only minor effects on behavioral outcome}

In addition to the histological and neurochemical analysis, mice were tested for their motor behavior in different motor exercises at two and four weeks after the intracranial injections.

To assess the functional impact of the 6-OHDA lesion on the nigrostriatal system, we performed the apomorphine turning test. The subcutaneously applied dopamine agonist apomorphine induces a hyperactivation of postsynaptic striatal dopamine receptors forcing lesioned mice to contralateral (ctl) rotational movements (turns). The number of turns a mouse completed was counted and could be used as an indicator for individual 6-OHDA lesion size. As expected, all intoxicated mice showed a strong contralateral rotation behavior after two weeks, while vehicle injected mice exhibited a naturally explorative behavior to both sides $($ LAA/PBS $=6.2 \pm 0.63 \mathrm{ctl}$ turns $/ 20 \mathrm{~min}$ and $6.9 \pm 2.17 \mathrm{ipl}$ turns $/ 20 \mathrm{~min}$; $6-\mathrm{OHDA} / \mathrm{PBS}=152.5 \pm 15.94 \mathrm{ctl}$ turns $/ 20 \mathrm{~min}$ and $1.9 \pm 0.87 \mathrm{ipl}$ turns $/ 20 \mathrm{~min}$; 6-OHDA/ROCK2-shRNA0.25 $=134.83 \pm 17.62 \mathrm{ctl}$ turns $/ 20 \mathrm{~min}$ and $0.75 \pm 0.39 \mathrm{ipl}$ turns $/ 20$ $\min ; 6-O H D A / R O C K 2-s h R N A 1.0=155.09 \pm 24.20 \mathrm{ctl}$ turns $/ 20 \mathrm{~min}$ and $2.27 \pm 0.70 \mathrm{ipl}$ turns $/ 20 \mathrm{~min}$; 6-OHDA/control-shRNA $=100.2 \pm 14.57 \mathrm{ctl}$ turns $/ 20 \mathrm{~min}$ and $1.4 \pm 0.7 \mathrm{ipl}$ turns/20 min; Figure 3.2.5-A, upper diagram). The comparison between the ROCK2-shRNA and the control-shRNA treated group resulted in no turning alteration.

In all groups injected with 6-OHDA a small increase in rotation behavior could be observed in the test after four weeks, however there was again no diminished turning number of AAV.ROCK2-shRNA injected mice detectable (LAA/PBS $=3.0 \pm 0.46 \mathrm{ctl}$ turns $/ 20$ $\min$ and $3.7 \pm 0.93 \mathrm{ipl}$ turns/20 $\mathrm{min} ; 6-O H D A / P B S=169.88 \pm 14.64 \mathrm{ctl}$ turns $/ 20 \mathrm{~min}$ and $0.25 \pm 0.23 \mathrm{ipl}$ turns/20 $\mathrm{min} ; 6-\mathrm{OHDA} /$ ROCK2-shRNA0.25 = $162.92 \pm 22.26 \mathrm{ctl}$ turns $/ 20 \mathrm{~min}$ and $0.08 \pm 0.08 \mathrm{ipl}$ turns $/ 20 \mathrm{~min} ; 6-\mathrm{OHDA} /$ ROCK2-shRNA1.0 $=165.82 \pm 26.12 \mathrm{ctl}$ turns $/ 20 \mathrm{~min}$ and $0.27 \pm 0.19 \mathrm{ipl}$ turns $/ 20 \mathrm{~min} ; 6-\mathrm{OHDA} /$ control-shRNA $=126.2 \pm 21.40 \mathrm{ctl}$ turns $/ 20 \mathrm{~min}$ and $0.5 \pm 0.47 \mathrm{ipl}$ turns/20 min; Figure 3.2/5-A, lower diagram). 
Another test to estimate unilateral lesion extent is the cylinder rearing test. The mouse natural exploration behavior was utilized to examine lateralized motor coordination at two and four weeks after 6-OHDA injection. In general, the unilateral lesion led to a lower frequency of contralateral forelimb usage to touch the cylinder walls during rearing. As expected, a significant increase of rears with using the right paw in comparison to the left paw was detected in unilaterally 6-OHDA-lesioned animals, due to the right striatal lesions and lower innervations of the left paw at two weeks after intoxication (Figure 3.2/5-B, upper diagram; 6-OHDA/PBS = right: $27.83 \pm 6.51 \%$ vs. left: $1.56 \pm 0.82 \%)$. Unlesioned animals were touching the walls with either the right or the left paw in equal amounts (LAA/PBS = right: $1.66 \pm 0.71 \%$, left: $1.68 \pm 0.69 \%$ ), but in general they used the single paws less often than the lesioned mice. The frequency of using the right paw more often than the left paw was not as pronounced in AAV.ROCK2-shRNA injected animals compared to lesion controls, however there was no difference to the control-shRNA group observable (6-OHDA/ROCK2-shRNA1.0 = right: $16.03 \pm 3.9 \% ; 6-O H D A / R O C K 2-$ shRNA0.25 = right: $14.94 \pm 4.23 \%$ vs. $6-O H D A / c o n t r o l-$ shRNA = right: $9.52 \pm 4.87 \%)$.

After four weeks, the cylinder test observations resulted in the same outcome, the 6-OHDA lesioned animals were using more often their right paws for touching the wall, than the left one and compared to unlesioned animals there was still a significant difference in paw using frequency (Figure 3.2/5-B, lower diagram; 6-OHDA/PBS = right: $15.42 \pm 2.85 \%$ vs. left: $2.82 \pm 1.81 \%$ vs. $L A A / P B S=$ right: $4.82 \pm 1.75 \%$, left: $4.89 \pm 1.91 \%)$. Treatment with AAV.ROCK2-shRNA seemed to have no beneficial effect on paw usage after four weeks.

To evaluate influences of AAV.ROCK2-shRNA treatment on motor endurance in 6-OHDA lesioned mice the rotarod test with accelerated speed of the running wheel over time was employed. Two weeks after intracerebral injections the motor performance of lesioned mice decreased significantly compared to the vehicle injected animals (Figure $3.2 / 5-C ; \quad L A A / P B S=196.1 \pm 14.56 \mathrm{~s}$ vs. $6-\mathrm{OHDA} / \mathrm{PBS}=149.63 \pm 11.78 \mathrm{~s})$. The rotarod performance of animals with 6-OHDA lesion was, however, not significantly different from animals injected with control- or ROCK2-shRNA. Therefore the treatment with ROCK2-shRNA seemed to have no improving effect compared to the control-shRNA injected group (6-OHDA/ROCK2-shRNA0.25 $=165.89 \pm 21.49$ s, 6 -OHDA/ROCK2-shRNA1.0 = $188.48 \pm 26.37 \mathrm{~s}$ vs. $6-\mathrm{OHDA} / \mathrm{control}-\mathrm{shRNA}=179.63 \pm 12.36 \mathrm{~s})$. At four weeks after the surgeries no lesion impairment for rotarod performance was observed between any groups. 
In order to detect more subtle changes in motor behavior, we employed the Catwalk $X T$ gait analysis system at two and four weeks after 6-OHDA-injection. Recording the paw prints from below, there were around 80 parameters compiled, considering each single paw, as well as the interlimb coordinations and walking patterns. Out of these parameters just a few quantifications showed alterations in paw statistics after unilateral 6-OHDA lesion compared to unlesioned animals. For example, mice injected with 6-OHDA showed in general bigger foot print areas as compared to the vehicle injected group after four weeks, especially the print width of the forelimbs increased significantly (LAA/PBS = right front: $0.59 \pm 0.06 \mathrm{~cm}$, left front: $0.6 \pm 0.05 \mathrm{~cm}$ vs. 6 -OHDA/PBS = right front: $0.77 \pm 0.03 \mathrm{~cm}$, left front: $0.78 \pm 0.05 \mathrm{~cm}, * * \mathrm{P}=0.008$; vs. $6-\mathrm{OHDA} / \mathrm{ROCK} 2$-shRNA0.25 = right front: $0.73 \pm 0.05$ $\mathrm{cm}$, left front: $0.72 \pm 0.03 \mathrm{~cm},{ }^{*} \mathrm{P}=0.042$; vs. $6-\mathrm{OHDA} / \mathrm{ROCK} 2-\mathrm{RNA} 1.0=$ right front: $0.73 \pm 0.03$ $\mathrm{cm}$, left front: $0.74 \pm 0.03 \mathrm{~cm}, * \mathrm{P}=0.039 ; 6-\mathrm{OHDA} /$ control-shRNA $=$ right front: $0.74 \pm 0.03$ $\mathrm{cm}$, left front: $\left.0.73 \pm 0.03 \mathrm{~cm},{ }^{*} \mathrm{P}=0.027\right)$. ROCK2-shRNA treatment revealed no significant impact on footprint areas, however.

Focused on the parameter describing the ratio of the maximal paw print intensity after initial paw contact with the glass plate in relation to the duration of the paw stand on the plate (i.e. Max Intensity At [\%] = (Max Intensity At (s)-initial contact)*100)/stand), we observed an increase for the right hind $(\mathrm{RH})$ paw in 6-OHDA lesioned mice, while on the contrary this parameter decreased for the left hind (LH) paw at four weeks after unilateral intoxication $(\mathrm{LAA} / \mathrm{PBS}=\mathrm{RH}: 37.42 \pm 2.0 \%$, LH: $37.26 \pm 2.5 \%$ vs. $6-\mathrm{OHDA} / \mathrm{PBS}=\mathrm{RH}$ : $48.7 \pm 5.9 \%$, $\mathrm{LH}: 28.7 \pm 2.5 \%$; Figure $3.2 / 5-\mathrm{D})$. Interestingly, animals treated with ROCK2shRNA showed a reduced Max Intensity At [\%] regarding the right hind limbs as compared to control-shRNA, reaching significance in the group treated with the high virus titer (6-OHDA/ROCK2-shRNA0.25 = RH: $37.8 \pm 3.17 \% ; 6-O H D A / R O C K 2-R N A 1.0=$ RH: $32.61 \pm 2.32 \%$ vs. $6-\mathrm{OHDA} /$ control-shRNA $=\mathrm{RH}$ : $41.96 \pm 3.17 \%$; Figure $3.2 / 5-\mathrm{D}$ ) at four weeks after lesion. Regarding the left hind limbs, Max Intensity At [\%] increased in ROCK2-shRNA injected mice, although this effect was not statistically significant compared to controlshRNA treated animals (6-OHDA/ROCK2-shRNA0.25 = LH: $35.88 \pm 3.62 \%$; 6-OHDA/ROCK2RNA1.0 = LH: $33.36 \pm 1.52 \%$ vs. 6-OHDA/control-shRNA = LH: $29.93 \pm 1.95 \%$; Figure 3.2/5-D). Therefore shRNA-mediated downregulation of nigral ROCK2 expression seems to influence this parameter, which is altered by the unilateral 6-OHDA lesion. 
A

Apomorphine turning test
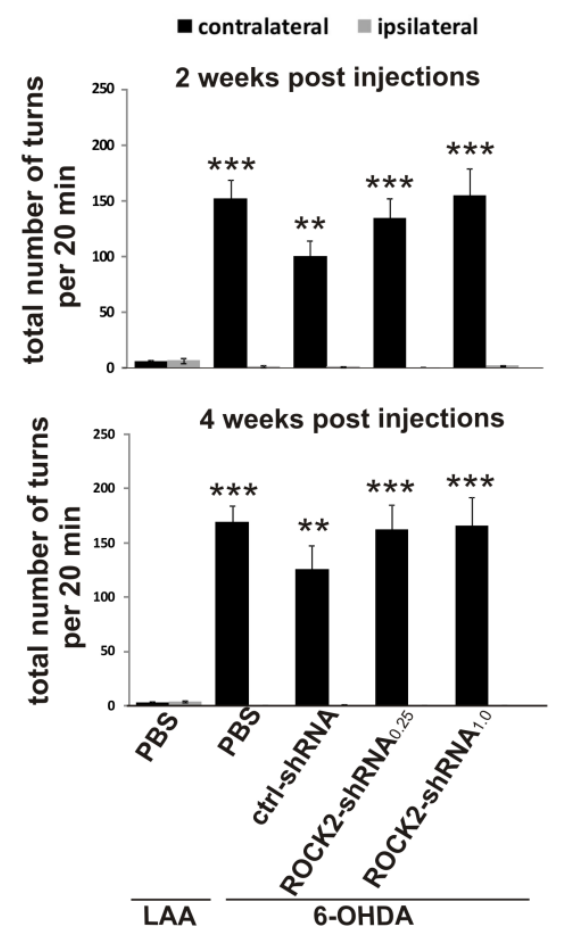

C

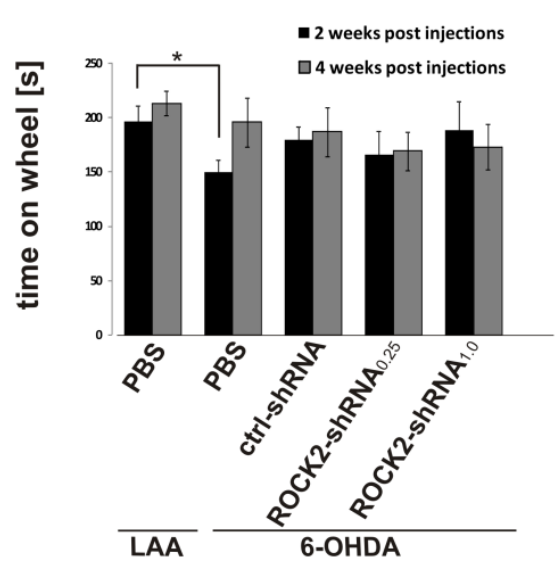

B

\section{Cylinder rearing test}
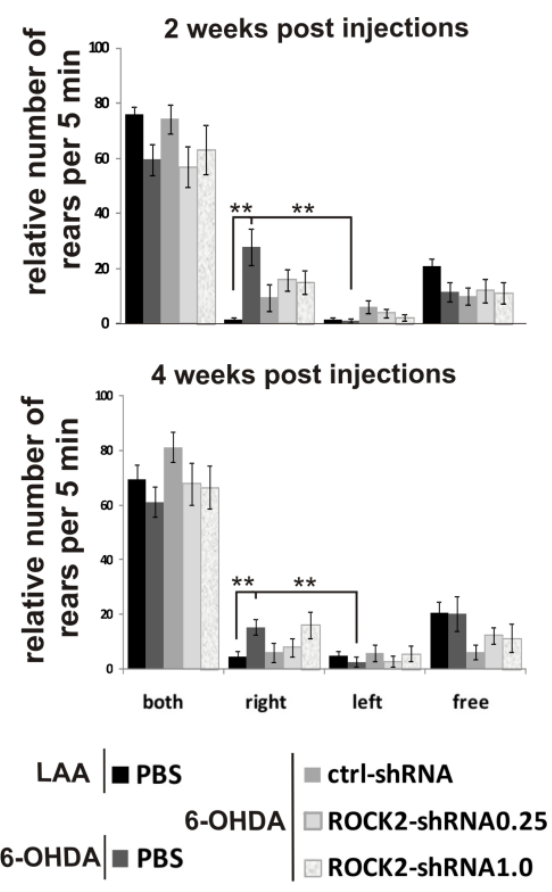

D

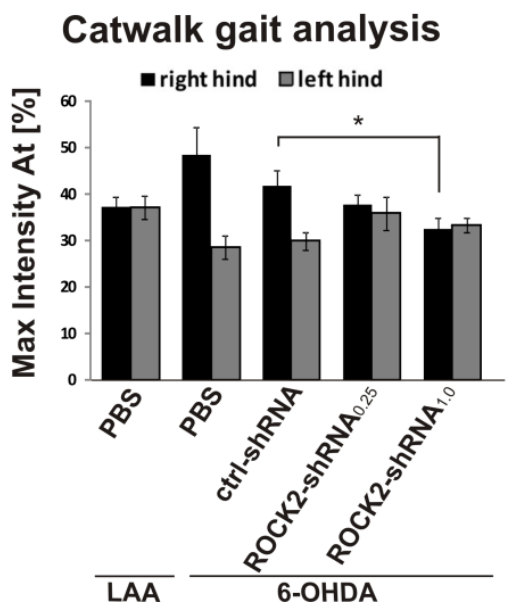

Figure 3.2/5: Analysis of motor behavior two and four weeks after 6-OHDA lesion and simultaneous transduction with AAV.shRNA. (A) Quantification of rotation behavior induced by apomorphine treatment at two different time points. Numbers of contra- and ipsilateral turns/20min after apomorphine injection with LAA/PBS ( $n=10), 6-O H D A / P B S, ~(n=8), 6-O H D A / c o n t r o l-s h R N A$ (ctrl-shRNA, $n=10), 6-O H D A / R O C K 2-s h R N A$ in a titer of $0.25 \times 10^{8} \mathrm{TU} / \mu \mathrm{l}$ (ROCK2-shRNA0.25, n=12), OHDA/ROCK2-shRNA in a titer of 1.0x 10 ${ }^{8} \mathrm{TU} / \mu \mathrm{l}$ (ROCK2shRNA1.0, $\mathrm{n}=11)$.

(B) Motor coordination was tested in the cylinder rearing test at two and four weeks after injections. Quantification of the relative number of rears against the wall with "both" paws, only the "right" or "left" paw or "free" rears. Groups analyzed were LAA/PBS $(n=10), 6-O H D A / P B S(n=10$ at two and $n=8$ at four weeks after lesion), 6-OHDA/control-shRNA (ctrl-shRNA, $\mathrm{n}=10$ ), 6-OHDA/ROCK2-shRNA in a titer of 0.25x 10 ${ }^{8}$ TU/ $\mu$ l (ROCK2shRNA0.25, n=12) and OHDA/ROCK2-shRNA in a titer of $1.0 \times 10^{8} \mathrm{TU} / \mu \mathrm{l}$ (ROCK2shRNA1.0, $\left.\mathrm{n}=11\right)$. 
(C) Analysis of motor endurance on a rotarod at two and four weeks after 6-OHDA and AAV.shRNA injection with groups as LAA/PBS ( $n=10), 6-O H D A / P B S ~(~ n=10$ at two and $n=8$ at four weeks after lesion), 6-OHDA/controlshRNA (ctrl-shRNA, n=10), 6-OHDA/ROCK2-shRNA in a titer of $0.25 \times 10^{8} \mathrm{TU} / \mu \mathrm{l}$ (ROCK2-shRNA0.25, $\mathrm{n}=12$ ), OHDA/ROCK2-shRNA in a titer of $1.0 \times 10^{8} \mathrm{TU} / \mu \mathrm{l}$ (ROCK2-shRNA1.0, $\mathrm{n}=11$ ).

(D) Catwalk XT gait analysis revealed significant differences in motor coordination in the parameter Max Intensity At [\%] at four weeks after the intracranial injections. Displayed groups are LAA/PBS ( $n=10)$, 6-OHDA/PBS ( $n=8), 6-O H D A / c o n t r o l-s h R N A$ (ctrl-shRNA, $n=10$ ), 6-OHDA/ROCK2-shRNA in a titer of $0.25 \times 10^{8}$ TU/ $\mu$ l (ROCK2-shRNA0.25, n=12), OHDA/ROCK2-shRNA in a titer of 1.0x $10^{8} \mathrm{TU} / \mu \mathrm{l}$ (ROCK2-shRNA1.0, n=11). Data for all behavioral tests are given as means \pm SEM with $* P<0.05, * * P<0.01, * * * P<0.001$ (One-way-ANOVA with Dunnett's test compared to control-shRNA or to vehicle group).

\subsection{Effect of long-term ROCK modulation on synaptic vesicle dynamics in vitro}

To further characterize the molecular role of ROCK in synaptic vesicle dynamics, mature primary hippocampal neurons were treated with the pharmacological ROCK inhibitor fasudil for 5 consecutive days. Via electrical field stimulation, cells were forced to execute transmitter release, which consists of fusion with the pre-synaptic membrane and the recycling of the vesicles by endocytosis. By imaging the amount of exo- and endocytosed vesicles the movement kinetics and released vesicle amounts after fasudil treatment were analyzed.

\subsubsection{Fasudil treatment has no negative effects on cell viability on DIV15}

To exclude toxic effects of fasudil application in a concentration of $20 \mu \mathrm{M}$ on primary hippocampal neurons after 5 DIV treatment, a toxilight assay was performed. Therefore the amount of adenylate kinase (AK), a protein released from dying cells, was measured in the medium of each sample and compared between the two groups for 3 independent cultures at DIV15 after 5 days of consecutive fasudil treatment (from DIV11-15). No increased toxicity after fasudil treatment was observed (Figure 3.3/1). 


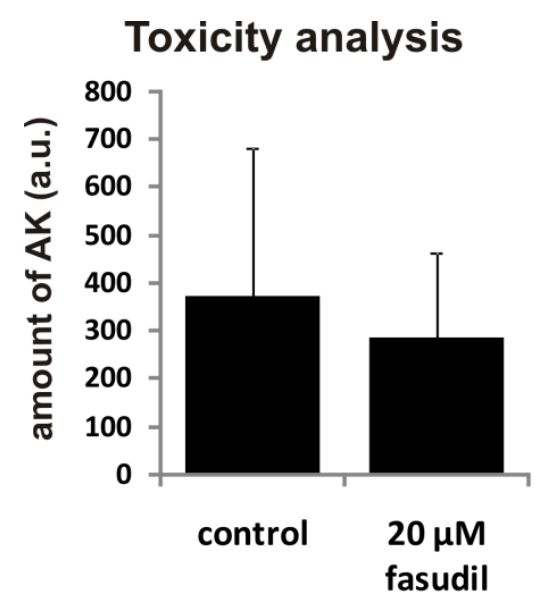

Figure 3.3/1: Analysis of toxicity after fasudil treatment for 5 consecutive days. The statistical evaluation resulted in no toxicity differences between the untreated and the fasudil treated condition $(n=3)$. Data are given as means \pm SEM (One-way-ANOVA with Dunnett's test compared to control).

\subsubsection{Pharmacological ROCK inhibition alters synaptic vesicle dynamics in vitro}

To investigate the molecular effects of pharmacological ROCK inhibition on synaptic vesicle dynamics, primary hippocampal neurons were cultured for 14-15 DIV to generate a mature culture system with a distinct synaptic network of neurons. Fasudil treatment was performed on DIV10 by adding the substance into the medium. By stimulating the cells with alternating electrical fields of $20 \mathrm{~Hz}$ for 30s transmitter release occured and the amount of membrane docking and recycled vesicles could be observed using an antibody-conjugated fluorophore (Atto647N), which is taken up into the cells via endocytosis. Fixation of the cultures after stimulation allowed the quantification of fluorescence intensity as a marker for synaptic activity. Negative controls were generated by incubating the cells in the presence of the antibody solution and the sodium channel blocker tetrodotoxin (TTX) on ice, thus the antibody only bound to antigens present on the cell surface. Acquisition of fluorescence intensity nicely illustrated less signal in not stimulated cells (Figure 3.3/2-A) compared to neurons, which underwent exo- and endocytosis of synaptic vesicles after electrical stimulation (Figure 3.3/2-C). Interestingly treatment with $20 \mu \mathrm{M}$ fasudil resulted in a $35 \%$ higher mean fluorescence signal on cell surface in non-stimulated conditions compared to controls (normalized to control $=1$ vs. fasudil $=1.35 \pm 0.11$ a.u., Figure 3.3/2-B), suggesting an effect of ROCK inhibition on the deposition of synaptotagmin1 (syt1) transmembrane proteins on the cell surface. 

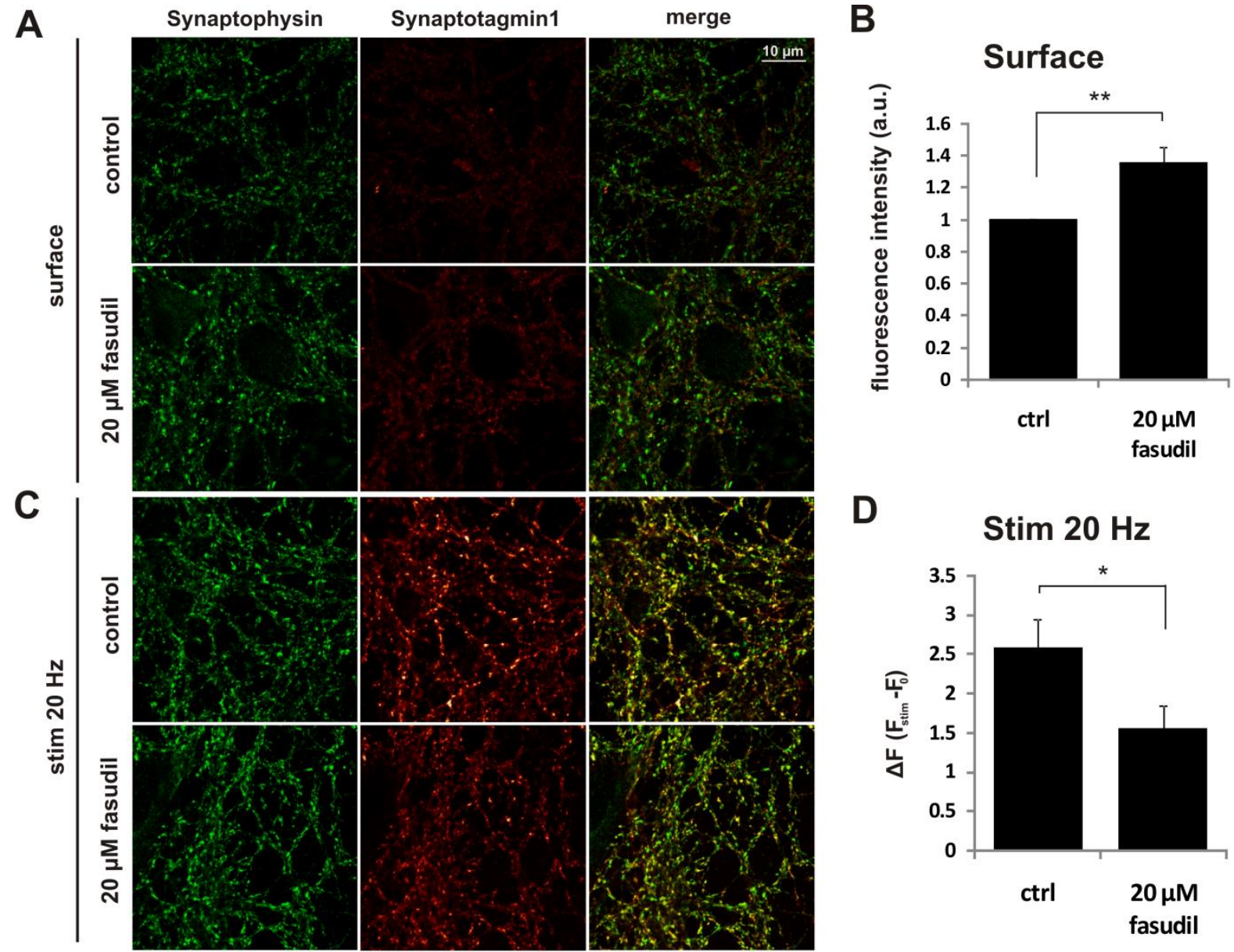

D

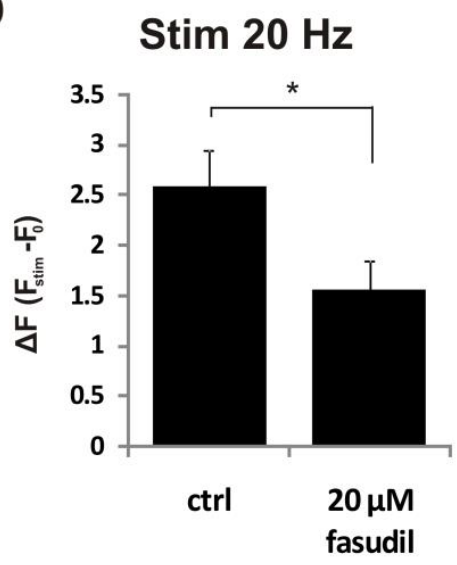

Figure 3.3/2: Analysis of vesicle dynamics from unstimulated and stimulated primary hippocampal neurons. Surface staining of control and fasudil treated cells (A) depicted an increase in synaptotagmin1immunoreactivity after ROCK inhibition (B). Electrical stimulation led to increased fluorescence signal in synaptophysin-immunoreactive synaptic boutons, indicating vesicle release and retrieval (C). After fasudil treatment there was a significant reduction in fluorescent vesicles, indicating attenuated vesicle dynamics (D), compared to controls. Data are given as means \pm SEM with $n=12$. ${ }^{*} \mathrm{P}<0.05,{ }^{*} \mathrm{P}<0.01$ (One-way-ANOVA with Dunnett's test compared to untreated control).

After electrical stimulation with $20 \mathrm{~Hz}$ over 30s, which led to action potential triggered exocytosis and recycling of synaptic vesicles, there was much more syt1 fluorescence signal in synapses without fasudil treatment (Figure 3.3/2-C, upper panel), while pharmacological ROCK inhibition seemed to decrease the amount of syt1 labeled vesicles (Figure 3.3/2-C, lower panel). The amount of recycled vesicles in synaptophysinimmunoreactive (synphy-IR) synaptic boutons after stimulation is represented by $\Delta \mathrm{F}$, which 
is the difference in fluorescence intensity $F$ after stimulation $\left(F_{\text {stim }}\right)$ and the surface signal without stimulation $\left(F_{0}\right)$. Comparing the fluorescence intensities from both conditions, there was a significant reduction in fluorescence change in the fasudil treated cultures to around $60 \%$ of $\Delta \mathrm{F}$ of the untreated cells, meaning that there was less vesicle release or retrieval after stimulation and a recycle time of 5.5 minutes (control $=2.58 \pm 0.37$ vs. fasudil $=1.56 \pm$ 0.28 , Figure 3.3/2-D). Taken together the pharmacological ROCK inhibition led to enhanced levels of syt1 in the cell membrane, while the amount of vesicles exocytosed after electrical stimulation and a recycling time of 6 minutes was significantly decreased compared to untreated controls.

Another way to investigate vesicle dynamics is the optical life imaging technique, where living cells are labeled with a dye, electrically stimulated and the cellular response is acquired simultaneously. The dye used is a $\mathrm{pH}$-sensitive Cy5-conjugated syt1 antibody (CypHer), in which cells are incubated 1-2 $\mathrm{h}$ before the experiment. During the incubation time the antibody is taken up into the vesicles of the neurons by spontaneous action potential driven exocytosis and recycling. Because of the acidic milieu of the vesicle lumen, the $\mathrm{pH}$-sensitive Cy5-fluorophore is glaring, while this fluorescence is quenched by exposition to the more alkaline extracellular tyrode buffer during exocytosis following stimulation. As a result of re-acidification of recycled vesicles, the fluorescence increases again. The pH-sensitivity of the fluorophore therefore monitors the synaptic vesicle dynamics in real time.

After stimulating the neurons with $20 \mathrm{~Hz}$ for $30 \mathrm{~s}$ the decrease in fluorescence signal turned into an increase of fluorescence starting after the electrical stimulation has finished. For each phase of stimulation and the resulting vesicle movements images were taken in an interval of two seconds and the averaged change in fluorescence from all synaptic boutons per field of view could be described for each measurement as a curve as it is illustrated in Figure 3.3/3-A. The descending course of the curves offered valuable cues about the vesicle kinetics during exocytosis after stimulation while the ascending part described the kinetics of membrane recycling and vesicle re-acidification. To estimate possible alterations in vesicle kinetics, exo- and endocytosis were analyzed separately by calculating the exponential fit for decay (exocytosis, Figure 3.3/3-B) or for rise to maximum (endocytosis, Figure 3.3/3-C) for each curve. Functions used to find the exponential fit were: $f=y 0+a * \exp \left(-b^{*} x\right)$ for exponential decay and $f=y 0+a * \exp \left(b^{*} x\right)$ for exponential rise. 
Next, the time constant $\tau$ was defined, describing the time an exponential decay needed to reach around $36.8 \%(1 / e)$ of the initial value. $\tau$ also specified the time an exponential rise required to reach around $63.2 \%$ of the maximum value. Comparing mean $\tau$ for fluorescence decay from the group treated with fasudil to the untreated controls resulted in no changes in the kinetics for exocytosis of synaptic vesicles (control $=7.59 \pm 1.06$ s vs. fasudil $=9.61 \pm 1.74 \mathrm{~s}$, Figure $3.3 / 3-D)$. The averaged time constant for the rise of fluorescence signal revealed no differences in kinetics of recycled vesiclesas well (control = $19.69 \pm 5.62$ vs. fasudil $=15.88 \pm 5.06 \mathrm{~s}$ ). Therefore fasudil treatment is not altering the synaptic vesicle kinetics during exo- and endocytosis, but affecting the amount of vesicles exocytosed after stimulation (see Figure 3.3/2-C+D).

A

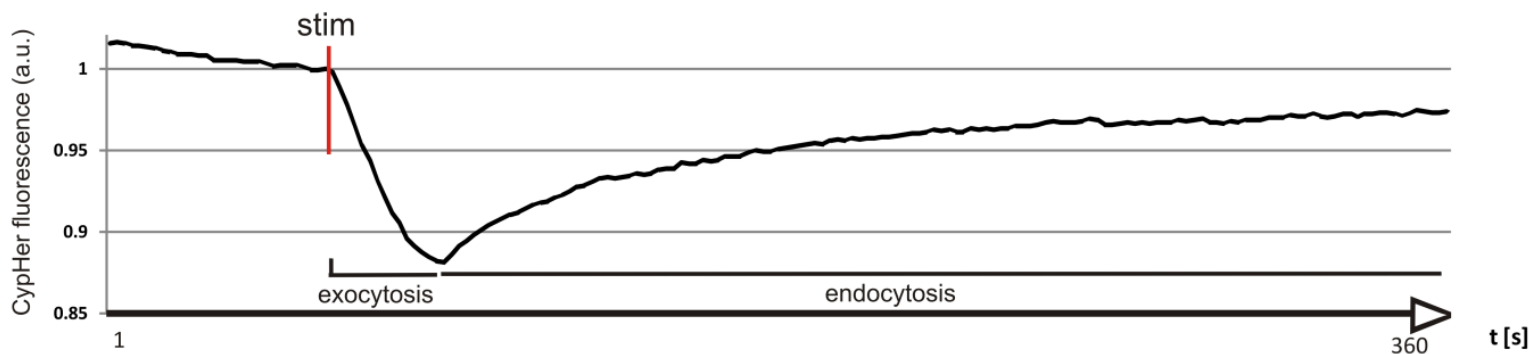

\section{B Exocytosis}
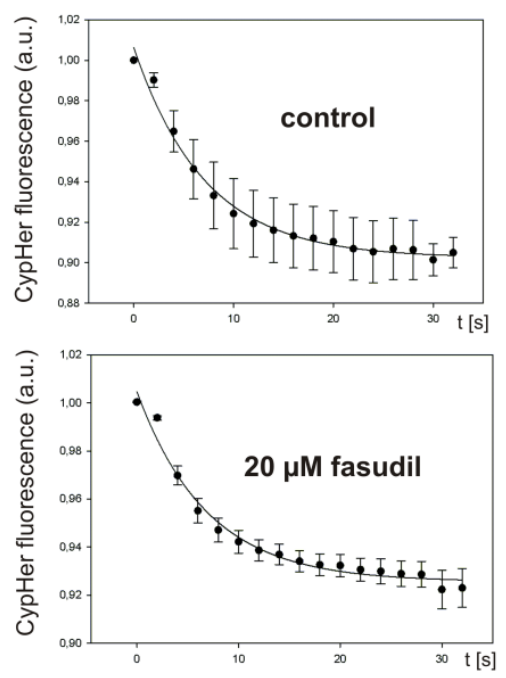

C Endocytosis
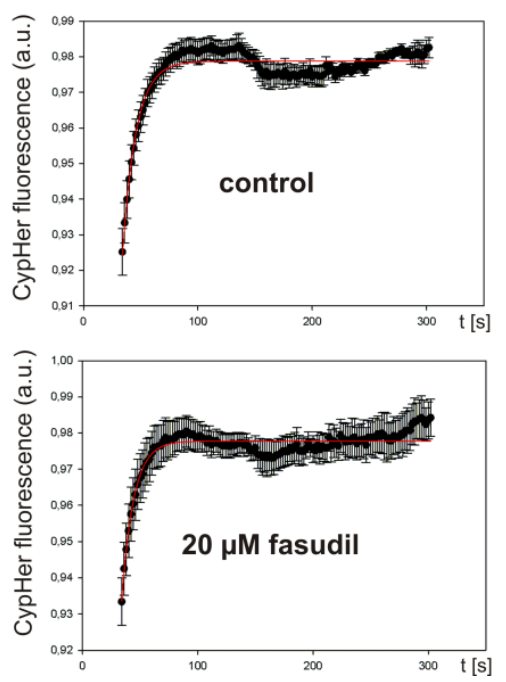

D

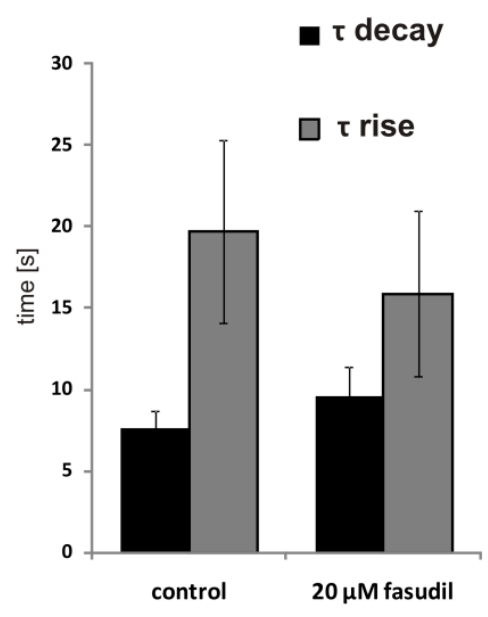

Figure 3.3/3: Analysis of exo- and endocytotic kinetics in primary hippocampal neurons after electrical stimulation. In A an exemplary curve of CypHer fluorescence change is shown related to the time. The descending part of the curve is describing exo- and the ascending part endocytosis. The averaged exponential fit from the mean fluorescent value per time point is illustrated as line in B for the control cultures (upper panel, $n=20$ ) and for fasudil treated cells (lower panel, $n=18$ ). Same is shown in $C$ for exponential rise, indicated with red line for untreated (upper panel, $n=15$ ) and for fasudil treated cultures (lower panel, $n=10$ ). From each 
exponential fit per measured view field $\tau$ was calculated but resulted in no statistically relevant changes, neither for exo- nor for endocytosis after fasudil treatment (D). Data are given as means \pm SEM (One-wayANOVA).

\subsubsection{Fasudil treatment leads to a shift in G-/F-actin ratio}

To understand the molecular mechanisms of how pharmacological ROCK inhibition via fasudil treatment is modulating the synaptic vesicle cycle in primary hippocampal neurons, the actin cytoskeleton - one of the main ROCK downstream targets - was further examined. The actin filaments build an intricate network, which interacts with vesicles at the active zone. Because ROCK is one of the key modulators for assembly and disassembly of filamentous actin, the effect of ROCK inhibition on the amount of filamentous F-actin and free globular G-actin was investigated. To this, the actin filaments were separated from G-actin by ultracentrifugation, than depolymerized into G-actin monomers and the amount of G-actin was evaluated by performing western blot analysis. Blotting of actin resulted in much higher band intensities for globular actin, compared to the amount of filamentous actin (Figure 3.3/4-A).

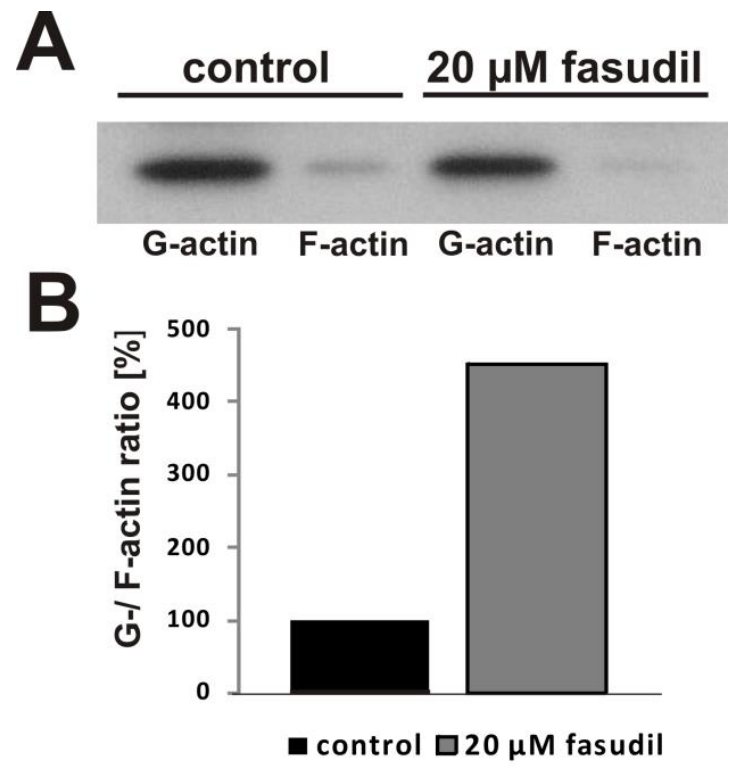

Figure 3.3/4: Western blot analysis of G- and F-actin amount in primary hippocampal neurons with and without fasudil treatment. Band intensities of actin indicated a strong shift to less F-and more G- actin in fasudil treated cells (A), confirmed by the quantification of actin band intensities and calculation of the G-/F-actin ratio (B, $\mathrm{n}=1)$. 
To neglect possible variability in total actin amounts, the G-/F-actin ratio was calculated. The quantification of band intensities showed a massive change in the G-/F-actin ratio revealing more globular and less filamentous actin in the fasudil treated sample (Figure 3.3/4-B). Unfortunately, because of problems in growing of the cell culture, there was only one culture available to evaluate the actin amounts. The analysis indicated a clear disassembly from F-actin to G-actin after ROCK inhibition via fasudil treatment, however has to be replicated in the future to validate this result. 


\section{Discussion}

The cure of neurodegenerative diseases is one of the main goals in today's neuroscience research. One of the main obstacles is the delayed diagnostics in relation to the molecular pathology. In Parkinson's disease (PD), for instance, the damage at the neuronal level is quite pronounced at the time when the first motor symptoms are recognized (Bernheimer et al., 1973). However, the complexity of normal cellular functions, its role in aging and during the generation of pathological features, still remains elusive.

In PD, several neuropathological mechanisms have been elucidated such as protein mutations, protein misfolding and aggregation, excitotoxicity, oxidative stress and glial infiltration. However, whether these mechanisms are cause or consequence still remains unknown (Dauer \& Przedborski, 2003; Dickson et al., 2009). There is a growing body of evidence that the inhibition of the ROCK pathway could prevent the progression of neuronal degeneration by antagonizing apoptotic cascades, stimulating intrinsic survival as well as facilitating neuronal regeneration in the affected neurons in vitro and in vivo (Raad et al., 2012; Tönges et al., 2011; Shi \& Wei, 2008). Additionally, ROCK inhibition influences the development of reactive astrogliosis and inflammation, thereby protecting neurons from degeneration (Lau et al., 2012; Villar-Cheda et al., 2012). All these beneficial properties of ROCK inhibition suggest that it is a promising candidate for the treatment of PD and other neurodegenerative disorders.

\subsection{Is there intrinsic regenerative capacity or a regeneration failure in Parkinson's disease?}

In order to estimate the potential impact of ROCK inhibition in PD patients there was the primary need to determine ROCK expression levels in the human brain. Additionally, the question arose whether the expression level of ROCK is altered in PD and if it thereby affects the disease progression or regeneration. On the other hand, the expression level of the growth-associated protein GAP-43 could indicate a potential regenerative capacity in the affected brain structures. Therefore, we investigated in post mortem tissue, whether alterations in the expression levels of ROCK2 and GAP-43 showed evidence for 
compensatory regenerative responses or if these proteins are potentially indicating a regeneration failure in PD. However, examination of proteins in human brains, from both control brains as well as from brains with neuropathology, always comes with the difficulty of a large interindividual variance. Therefore, studies of human brain tissue are more difficult to standardize than evaluations of well-controlled animal tissue and have to be interpreted with caution.

\subsubsection{The investigated brain sections mirror characteristic features of PD}

The immunohistochemical analysis of $\mathrm{TH}$ in the present brain sections confirmed that in the majority of the PD cases, there was a pronounced degeneration of dopaminergic neurons. However, the TH signal in the striatum depicted that several individuals from the age-matched control group (non-PD) also seemed to have a lower striatal dopaminergic fiber density as compared to young controls. This confirmed findings from other groups, who showed a linear decline in striatal dopaminergic projections during healthy aging by studying the abundance of dopamine transporters (DAT) in the striata of people in the age range from 18 to 88 using PET scan and SPECT imaging (Volkow et al., 1990; van Dyck et al., 2002).

On the other hand, our study did not show dopaminergic neuron loss in the substantia nigra (SN) in individuals of either the young or the age-matched control group in contrast to PD cases. This coincides with data from the recent literature showing that neuronal cell death is not a characteristic feature of healthy aging (Morrison \& Hof, 1997; Seibyl et al., 1995; Pakkenberg, 2004). Concerning our data on TH-immunoreactivity (TH-IR), there seemed to be a trend towards selective vulnerability of dopaminergic terminals in the striatum already in the aged brain, while the dopaminergic cell bodies were still unaltered. In PD cases the nigral dopaminergic cell death was quite pronounced, representing one of the main pathoanatomical characteristics of PD, but it was not reflected by significant decline in TH-IR fibers in the striatum. Thus, the decrease of dopaminergic neurons in the SN seems to be less age-related, while there is evidence for an age-depended decline of dopaminergic terminals, which is much more pronounced in PD disease patients. This supports the notion of a retrograde axonal degeneration and argues in favour of a stronger susceptibility of dopaminergic terminals. 
One characteristic accompaniment of neurodegeneration is the generation of glial scar tissue (Maragakis \& Rothstein, 2006). Once CNS damage occurs, astrocytes are engaged to seclude the injury side from healthy tissue (Silver \& Miller, 2004), which, however, constitutes simultaneously the major impediment to neuronal regeneration (Yiu \& Zhigang, 2009). Interestingly, studies from Mirza and colleagues (Mirza et al., 2000) did not reveal changes in astroglial reaction (GFAP-IR) in the SN or the striatum in PD cases compared to age-matched controls. In our analysis, we obtained similar results showing no differences in the GFAP amount of age-matched controls and PD cases in the SN. The addition of a young control group illustrated that there seems to be an age-related astrocytic proliferation in the $\mathrm{SN}$, which is not reaching significant levels and is not intensified in PD cases. On the contrary, regarding the GFAP-IR amounts in the striatum, the analysis of young controls showed almost no presence of astrocytes, while age-matched controls and PD patients depicted abundant GFAP-IR expression. From our point of view, there seems to be an age-dependent proliferation of activated astrocytes in the $\mathrm{SN}$ as well as in the striatum, which might be caused by age-related sporadic neuronal impairments (Morrison \& Hof, 1997; Pakkenberg, 2004). However, it is not clear, if the striatal dopaminergic fiber loss can be correlated to higher amounts of astrocytic proliferation, which might transform to detrimental reactive astroglia, or if this in turn is a consequence of degeneration.

Interestingly the immunohistochemistry against the microglial marker lba1 depicted no relevant differences in the presence of miroglia in the striatum of PD cases compared to controls. There are rather a few single individuals featuring a high amount of Iba1-IR in the young control and the PD group, suggesting a specific micoglial reaction and transient proliferation to undefined cues in the respective cases. However, not primarily the amount of microglia is important, but their state of activation (Barcia et al., 2012). PET studies of Ouchi and colleagues revealed a correlation of dopaminergic cell loss and the increase of activated microglia in the basal ganglia of PD patients (Ouchi et al., 2005). Therefore, in the case of neuronal tissue damage, activated or phagocytic active microglia may deliver trophic factors and remove cell debris as a requirement for tissue restoration (McGeer \& McGeer, 2008). Then again, proinflammatory cytokines decrease the beneficial phagocytic activity and commit macrophages to an inflammatory response (Hanisch \& Kettenmann, 2007; Hunter et al., 2007). Thus, by staining microglia in human brains with an antibody against Iba1, merely indicates possible activation and transient proliferation of microglia into 
damaged tissue, but gives no further information about their beneficial or detrimental features, mirroring only the cellular composition in the hours of death.

Taken together, the immunohistochemical characterisation of the present human brain sections from PD cases, young and age-matched controls confirmed the dopaminergic degeneration in the nigrostriatal system during PD. This was accompanied by striatal astrogliosis that in turn seemed to be an age-related feature, raising the idea of constituting a prerequisite for the development of PD. Nonetheless, the interindividual variances did complicate the unification and the interpretation of the results, which are generated from averaged values per group.

\subsubsection{GAP-43 and synaptophysin expression are altered in the nigrostriatal system in PD}

In order to determine the general regenerative capacity of the nigrostriatal system, the expression level of the growth associated protein GAP-43 was examined in the brains of PD cases, young and age-matched controls. Due to the fact that the striatal TH-labeling resulted in a diffuse tissue staining, TH-positive fibers could not be clearly separated. Therefore co-labeling of GAP-43 and TH-IR fibers was not feasible and thus, the total striatal GAP-43 protein expression was analyzed. GAP-43 is associated with the neuronal development and is mainly present in neuronal growth cones and immature synapses, however levels decline precipitously by the completion of mature synaptic networks (Frey et al., 2000; Neve et al., 1988). Interestingly, the immunohistochemical analysis of GAP-43 revealed quite distinct expression levels with heterogeneous distribution in the human striatum. Comparing the protein levels between PD cases and controls resulted in no expression differences between the groups. Therefore, dopaminergic degeneration seems to have no influence on GAP-43 expression levels in the striatum of PD patients, indicating no compensatory sprouting from adjacent neuronal tissue. This observation goes in line with findings from Iwata and colleagues employing the 6-OHDA rat model, where striatal intoxication and dopaminergic degeneration was not followed by changes in the GAP-43 protein expression (Iwata et al., 2001). Taking into account the results from a parallel study with Dagmar Galter from the Karolinska Institute in Sweden, there were also no detectable changes in the striatal GAP-43 mRNA in situ signal of PD cases and controls (manuscript in 
preparation). Thus, we assume that the degeneration of dopaminergic terminals is not reflected by changes in GAP-43 expression. However, the dynamic processes of neuronal plasticity or reorganisation and sprouting could also imply a short-term up-regulation of GAP-43, suggesting a transient increase as a consequence of dopaminergic degeneration. Therefore, transient changes in expression could not have been detected in the present analysis of post mortem tissue, where dopaminergic degeneration started a long time before the observation. Interestingly, the averaged synaptophysin immunoreactivity (synphy-IR) appeared unchanged in PD cases as well, even though there were quite high interindividual differences in the PD group. In two of the examined PD cases the drastic reduction of synaptophysin-IR could indicate a constant decrease of the synaptic compartment, caused by the degeneration of dopaminergic synaptic connections. Therefore, further analysis of a specific dopaminergic synaptic marker, such as the vesicular monoamine transporter (VMAT), could be used to determine the decline of especially striatal dopaminergic synapses (Miller et al., 1999; Okamura et al., 2010).

The evaluation of GAP-43 protein expression in the SN revealed only a sparse immunofluorescent signal in co-labeled TH-IR neurons. This finding was not surprising, because GAP-43 protein expression is assumed to be likely present in the neuronal terminals. Nevertheless, the trend of decreased GAP-43-IR signal in dopaminergic neurons of PD cases goes in line with findings from Dagmar Galter, who provided evidence for significantly decreased GAP-43 mRNA expression levels in dopaminergic neurons of PD patients. She demonstrated that in most of the remaining dopaminergic neurons of PD cases the GAP-43 mRNA signal was virtually absent, while controls exhibit GAP-43 mRNA signal in different amounts (manuscript in preparation). Same, Iwata and colleagues found out by studying GAP-43 mRNA amounts in dopaminergic neurons after intrastriatal 6-OHDA intoxication in rats, suggesting that the lack of GAP-43 mRNA expression resulted from neuronal damage and restricted cellular functioning of the transcription machinery (Iwata et al., 2001).

Furthermore, we could observe a significantly decreased synaptophysin-IR in the SNpc in PD cases constituting nigral presynaptic disintegrity, which might be caused by the distinct death of postsynaptic neurons. Interestingly, this decrease in presynaptic protein seemed to be an apparent feature in age-matched controls as well, however not reaching significant levels. In turn, this finding coincides with what is known from the present 
literature, confirming that characteristic features of aging are not represented by neuronal cell death. Age dependent synaptic changes are rather composed by region-specific loss in dendritic branching and spine density, probably caused from the higher vulnerability of certain cell types to environmental changes (Burke \& Barnes, 2006; Morrison \& Hof, 1997).

Taken together the decrease in GAP-43 protein revealed mild indication for impaired intrinsic regenerative capacity of dopaminergic neurons, while the decline of GAP-43-IR structures in the adjacent nigral neuropil seemed to be an age-related feature. Additionally, the presynaptic decline in the SN appeared to be age-dependent, however its significant diminution was restricted to PD.

\subsubsection{There is diffuse ROCK2 expression in the nigrostriatal system of the human brain}

In the present study, we demonstrated for the first time that there is ROCK2 expression in the human nigrostriatal system. The evaluation of ROCK2 immunoreactivity (ROCK2-IR), however, revealed only sparse expression throughout the SN and the striatum. Next to a weak diffuse tissue staining, ROCK2-IR occurred in conspicuous puncta-like structures that seemed to be distributed intracellularly and in the extracellular matrix of the tissue. The same punctate ROCK2 staining González-Forero and colleagues described to be present in hypoglossal motoneurons of rats, suggesting that ROCK2 is primarily expressed in neurons (Gonzalez-Forero et al., 2012). However, in the SNpc of human brains we could not allocate the ROCK2-IR to specific cell types or particular localisation in the cells. Measuring the ROCK2-IR in dopaminergic neurons of the $\mathrm{SN}$ resulted in no changes in the expression levels between PD cases and both control groups. Approximately the same averaged ROCK2 amounts were quantified in the nigral neuropil, showing no evidence for a different expression pattern in PD cases or age-matched controls. These findings suggest that the mean expression of ROCK2 is not altered in the SNPC in PD, at least not at the time point of death. Therefore there seems to be no elevated inhibitory signalling, at least not propagated through the ROCK2 pathway, which could be responsible for regenerative failure. However, there was one single case from the age-matched group that showed a noticeable high amount of ROCK2 expression in the nigral neuropil. Having a closer look at ROCK2 expression in the striatum, the overall analysis depicted that the same case expressed ROCK2 in very 
weak amounts in the putamen in comparison to the other age-matched controls. On the contrary, there was one PD case, which possessed very high ROCK2-IR in the putamen. These outliers suggest that ROCK2 expression might be a very dynamic process, which is up- or downregulated by particular external or internal cues. Consulting the literature to ROCK2 expression after neuronal lesion in humans, there is evidence for the upregulation of the ROCK upstream regulator Rho $(A+B)$ directly after traumatic brain injury, suggesting presumable involvement of the ROCK2 signalling cascade as a consequence of neuronal damage (Brabeck et al., 2004). However, the expression level alone cannot directly be correlated to kinase activity. The ROCK2 activity state attributes to either be inactive with perinuclear localization or to be translocated to the plasma membrane upon Rho activation (Shi \& Wei, 2008). In the present study of human brain tissue, ROCK2 expression could not be allocated to a particular localization. Thus, there seems to be no correlation between ROCK2 expression and advanced PD or aging.

\subsubsection{ROCK2 expression is altered in striatal astrocytes and microglia}

Although there were high interindividual disparities, the quantification of single striatal cells, which revealed increased ROCK2-immunofluorescent signal, indicate a strong evidence for the upregulation of ROCK2 expression in particular cells in the striatum of PD cases. Interestingly, this phenomenon was also quite pronounced in age-matched controls, however not reaching significance levels. We can assume that in PD and aging there are elevated numbers of cells showing highly expressed ROCK2, potentially correlated with enhanced ROCK2 activity. Interestingly, it turned out that in the group of PD cases these cells seemed to be primarily astrocytes and microglia, contradicting the assumption of GonzàlezForero and colleagues, that ROCK2 is predominantly expressed in neurons of the vertebrate brain (Gonzalez-Forero et al., 2012). The relationship between increased ROCK2 and the higher number of astrocytes in the striatum of PD cases remains ambiguous and reinforces the role of glia in PD. Therefore, it is not clear whether an abnormal activation of the ROCK2 pathway in astroglia occurs in PD or if this ROCK2 upregulation is due to transient cellular alterations to modulate the cytoskeleton, cellular migration and proliferation via the ROCK pathway. Because the age-matched controls exhibited the same trends in numbers of ROCK2 upregulating astrocytes, this phenomenon seemed to be age-related as well, however being 
intensified in PD. The similar finding of exhibiting a trend towards increased numbers of ROCK2-IR microglia in PD cases confirms the strong suspicion of involvement of both glial types in disease progression. For example, Barcia and colleagues demonstrated in a rat model, that MPTP intoxication enhanced ROCK activity, which in turn is associated with the activation of microglia cells, characterized by morphological changes (Barcia et al., 2012) and increased microglial ROCK2 expression (Villar-Cheda et al., 2012). Upregulated ROCK2 expression therefore provides evidence for an inappropriate microglial activity in PD patients.

In this context and in finding treatment strategies to arrest the progressive dopaminergic cell death or to facilitate nigrostriatal restoration in PD, pharmacological ROCK inhibition would become highly important. It is already known that ROCK inhibition exerts beneficial effects on brain injuries by attenuating glial scarring (Mueller et al., 2005). However, to what extent the infiltration of reactive astrocytes, the so called astrogliosis, or the final scar formation might be deleterious for neurons, remains elusive. Moreover, in lesion paradigms, astrocytes are known to constitute a physiological wall, therefore being partially responsible for the regeneration failure (Silver \& Miller, 2004). Investigations by Lau and colleagues about the effects of ROCK inhibition on cultured astrocytes showed that pharmacological ROCK inhibition evoked transcriptomic changes and cytoskeletal reorganization, which are consistent with astrocytes that adopted a physiologically beneficial phenotype. Furthermore, the expression levels of the brain derived neurotrophic factor (BDNF) or the excitatory amino acid transporter (EAAT) where upregulated, while the expression of the semaphorins SEMA4B and SEMA5A as well as the ephrin/Eph complexes ephrin-A/EphA and ephrin-B/EphB, which are supposed to transmit inhibitory guidance cues to neurons (Goldberg et al., 2004; Goldshmit et al., 2006), were dramatically decreased (Lau et al., 2012; Lau et al., 2011). Investigations from our group in the SOD1-G93A mouse model of ALS showed significantly reduced astroglial scar tissue in the affected area of the CNS after oral fasudil treatment compared to untreated animals at an intermediate time point during disease progression (Tönges et al., 2014). Furthermore, the activated microglia infiltration was significantly increased in the same fasudil treated animals and was considered to be involved in neuroprotective activities in the early stages of the disease (Tönges et al., 2014; Henkel et al., 2009). This was confirmed in a cytokine release paradigm in vitro, where ROCK inhibition via fasudil treatment led to a pronounced attenuation in the 
release of inflammatory cytokines and chemokines from cultured microglia, which were stimulated by the addition of lipopolysaccharides (LPS) (Hunter et al., 2007; McGeer \& McGeer, 2008; Zhao et al., 2011). In addition, by employing the MPTP mouse model of PD, Barcia and colleagues discovered that activated microglia formed intercellular contacts with the damaged dopaminergic neurons, involving reorganisation of the actin cytoskeleton and are thereby enable to engulf the damaged dopaminergic neuron, suggesting the formation of a microglial phagosome. They could show that ROCK inhibition blocked microglia migration and thereby prevented possible unnecessary elimination of attacked dopaminergic neurons by inhibiting the microglial cytoskeletal rearrangement (Barcia et al., 2012).

Taken together, we could show that dopaminergic degeneration is accompanied by a decrease in GAP-43 and synaptophysin protein expression in the neuropil of the SNpc in PD cases, however, this phenomenon was also present in age-matched controls. Additionally, there is strong evidence for the involvement of increased ROCK2 expression in astrocytes and microglia during PD progression. Furthermore, it seems that the diminution of synaptic plasticity and neuronal regenerative capacities, as well as the upregulation of glial ROCK2 expression in the striatum are age-related processes, which might be accelerated in PD. However, this study could not clarify whether such changes in protein expression are involved in the pathological process or are merely a consequence of dopaminergic degeneration, due to the fact of mirroring only the state of protein expression and cell composition in the terminated hours of life, disregarding fluctuations weeks, months, or years before. Nevertheless, the results from the present study, together with the knowledge about the role of ROCK inhibition on neuronal survival, regenerative outgrowth and in modulating glial formation, foster the role of pharmacological ROCK inhibition in the treatment of PD. 


\subsection{The role of neuronal ROCK inhibition in the 6-OHDA mouse model of PD}

The pharmacological inhibition of ROCK emerged as being a promising target in the treatment of different aspects linked to neuronal survival, regeneration and modulating glial infiltration in diverse models of neurodegeneration. Advantages of the ROCK inhibitor fasudil comprise the possibility that it can be applied orally, crossing the blood brain barrier and reach virtually all cell types in the nervous tissue (Mueller et al., 2005; Tatenhorst et al., 2014). Nevertheless, one drawback of the application of this kinase inhibitor is its incomplete specificity for ROCK2, inhibiting both ROCK isoforms and other kinases (Davies et al., 2000; Mueller et al., 2005). To ascribe the protective properties specifically to the inhibition of ROCK2 and to exclude effects from blocking other kinases, we used a virusmediated RNA-interference approach by unilateral expression of a small hairpin RNA (shRNA) in the SN of 6-OHDA intoxicated mice. The usage of vectors containing the neuron specific human synapsin promoter for the expression of the fluorophore and the unspecific polymerase $\mathrm{H} 1$ promotor for the transcription of the ROCK2-shRNA enables to identify transduced neurons by the expression of the fluorophore. This expression was confirmed to be exclusively neuronal by immunohistochemistry. By employing the AAV2 serotype, ROCK2shRNA expression was targeted preferentially to neuronal cells, which therefore nearly excluded effects of kinase inhibition from glia cells (Tenenbaum, 2004; Bartlett et al., 1998). It is thus important that the observed effects are attributed almost exclusively to ROCK2downregulation in neurons of the SNPc. The effective knockdown of the target protein ROCK2 with the used vector was previously confirmed by Western blot analysis of infected primary midbrain neuronal cultures (Saal et al., 2015) as well as by real-time PCR of infected cultured retinal ganglion cells (RGC), resulting in 75\% reduction of the ROCK2-mRNA after 7DIV and in vivo by immunohistochemistry against ROCK2 in RGC at two weeks after intravitreal virus injection (Koch et al., 2014).

To evaluate the protective effects of neuronal ROCK2 downregulation, we chose the 6-OHDA striatal lesion model to induce progressive retrograde degeneration of the dopaminergic nigrostriatal projections, which best resembles the progression of PD (AlvarezFischer et al., 2008). Viral vectors were injected simultaneously with 6-OHDA to mimic a therapeutic approach during the disease progression. Employing the 6-OHDA model offered the possibility to spatially separate AAV-mediated ROCK2 inhibition in nigral dopaminergic 
somata from the toxin-induced degeneration of striatal dopaminergic terminals. The observation time period of four weeks enabled the advanced dopaminergic impairment by anticipating spontaneous regeneration (Walsh et al., 2011; Schwarting \& Huston, 1996) distinguishing the effects of ROCK2 inhibition on neuronal degeneration.

\subsubsection{ShRNA mediated ROCK2 downregulation increases dopaminergic survival after 6-0HDA intoxication}

Four weeks after the injection, stereologically obtained numbers of TH-positive neurons in the SN of the non-intoxicated vehicle control group were similar to the results previously obtained in the non-intoxicated vehicle control groups of the 6-OHDA model (Tatenhorst et al., 2014) and of the MPTP model (Tönges et al., 2012). Therefore we conclude that the mechanical damage caused by the nigral injections alone is negligible. Since previous reports suggested toxic effects by long-term expression of shRNA-vectors in vivo (Blits et al., 2010), two AAV dosages were compared. Interestingly, the lower titer (0.25x $\left.10^{8} \mathrm{TU}\right)$ was more effective in attenuating 6-OHDA-mediated dopaminergic neuronal death than the higher titer $\left(1.0 \times 10^{8} \mathrm{TU}\right)$. In contrast to previous studies of our group, where oral treatment with fasudil showed no protective effect on TH-IR cells after 6-OHDA lesion (Tatenhorst et al., 2014), we could, in the present study, definitely assign the attenuated dopaminergic cell loss to the intrinsic shRNA-mediated ROCK2-inhibition. Although we did not observe overt toxicity, it is possible that the higher titer AAV.shRNA exerted unspecific toxic or off-target effects, which attenuated the protective effects of ROCK2-inhibition. Li and colleagues could show that activated ROCK is directly phosphorylating PTEN (Li et al., 2005), thus we can assume that specific shRNA mediated inhibition of ROCK2 is enhancing dopaminergic survival presumably through the PTEN/Akt/mTOR pathway (Tönges et al., 2011; Kim et al., 2011). A previous study from our group showed, that shRNA mediated ROCK2 downregulation increased cell survival and decreased axonal degeneration of RGCs in the rat optic nerve crush model with the same viral constructs as used in the present study (Koch et al., 2014). Here we could assign the beneficial effects to the activation of the Akt pathway and through the decreased activity of the protease caspase-3, which is an essential player in the apoptosis cascade (Porter \& Jänicke, 1999). 
Quantification of Nissl-positive cells revealed that the declined number of neurons was similar to the loss of TH-IR cell numbers. This indicated that 6-OHDA induced a specific dopaminergic cell death and we thus could assume that there is no misleading downregulation of TH as a consequence of 6-OHDA effects (Georgievska, 2002). While the histological quantification of striatal TH-IR fibers still identified $\sim 40 \%$ remaining axons in the lesion control, as compared to the vehicle injected animals at four weeks after lesion, the levels of dopamine and its metabolites in the HPLC analysis were reduced more severely. The significant fiber loss of approximately $60 \%$ corresponds to the amount of nigrostriatal degeneration in PD when the first motor symptoms manifest (Blum et al., 2001). However, in the present study, it seemed that there was a strong trend in attenuated dopaminergic fiber loss from shRNA-mediated ROCK2 downregulation, which was not significantly increased in comparison to the adequate shRNA control. Interestingly, in case of the low virus titer the fiber degeneration was just significantly alleviated in comparison to the 6-OHDA lesion only control $\left({ }^{*} p=0.05\right.$; because this is not the adequate vector control, we did not add this information to the results). This indicates an attenuation of the 6-OHDA effect propagated through intracellular ROCK2 downregulation, leading to a mild preservation of axon terminal morphology and of TH integrity in ROCK2-shRNA injected mice. However, this finding did not translate into similarly increased levels of dopamine or its metabolites HVA and DOPAC, which references to advanced impairments of terminal functionality caused by 6-OHDA. Similar results were found in our previous study by having a strong decline of dopamine and its metabolites four weeks after 6-OHDA lesion and consecutive oral fasudil treatment (Tatenhorst et al., 2014). In the study from Kim and colleagues the direct modulation of the survival cascade propagated through mTOR by intranigral injection of AAV-mediated Rheb (activator of mTOR) expression in the 6-OHDA mouse model, resulted in enhanced survival and preservation of the dopaminergic terminals and their functionality. They showed that the striatal TH-density and levels of dopamine and HVA after 6-OHDA lesion were virtually completely preserved, however virus mediated gene expression was started three weeks before the lesion (Kim et al., 2011). In contrast to our study, the Rheb gene expression was strongly increased at the time point of intoxication, which can explain the pronounced effects. In our experiments, the simultaneous injection of 6-OHDA and the ROCK2-shRNA lead to delayed expression of the shRNA (Koch et al., 2011; Shevtsova et al., 2005), while the 6-OHDA impact on the dopaminergic neurons is immediate. It is likely that this deferred 
downregulation of the target gene results in delayed activation of survival promoting pathways, consequently leading to advanced toxic effects on dopaminergic neurons. Taken together, this indicates that shRNA-mediated ROCK2 downregulation intrinsically protected the remaining neurons from degeneration. This would explain the morphological maintenance of dopaminergic terminals, while concurrently there is still functional impairment (Georgievska, 2002).

\subsubsection{Neuronal ROCK2 downregulation has only minor effects on behavioral outcome}

The total dopamine depletion observed with HPLC was additionally mirrored in the apomorphine turning test. The strong rotation behavior induced by the apomorphine injection in all 6-OHDA-lesioned animals at two weeks after lesion was even more pronounced after four weeks. This implies a further upregulation of striatal postsynaptic dopamine receptors to compensate for the lack of signal transduction resulting from the deleted dopamine transmission in all 6-OHDA lesioned animals (Schwarting \& Huston, 1996). As a consequence of the intraneuronal failure of shRNA-mediated downregulation on the preservation of dopaminergic functionality, ROCK2 inhibition could not attenuate rotation behavior after apomorphine application. Furthermore, behavior analyses with rotarod or the cylinder-rearing test were not significantly improved in animals treated with ROCK2-shRNA.

The effects of the 6-OHDA lesion were also examined using the Catwalk XT gait analysis system. The high sensitivity of this method has been validated previously by Vandeputte and colleagues, however they could observe only one significant change in the parameter for intensity of the front paws after striatal unilateral 6-OHDA lesion in rats (Vandeputte et al., 2010). In the present study we could show that the 6-OHDA lesion affected gait of mice, reflected in an increased paw print size, possibly to compensate postural instability. Simultaneously, the parameter giving the time interval to reach the maximal paw print intensity related to the duration of the stand (Max Intensity At), showed converse results regarding the hind paws. This finding indicates an irregularity in walking pattern of unilateral striatal lesioned mice concerning the hind limb, which was innervated by the lesioned brain hemisphere, as it has been described before in rats (Hsieh et al., 2011; Vandeputte et al., 2010). This step irregularity was completely rescued in the mice, which 
were injected with ROCK2-shRNA $\left(1.0 \times 10^{8} \mathrm{TU}\right)$. Thus, we could detect a significant reduction of 6-OHDA-induced motor impairment by ROCK2-shRNA compared to control-shRNA despite the lack of significant recovery in striatal levels of dopamine and its metabolites.

Taken together, it has to be acknowledged that the specific targeting of ROCK2 knockdown in the 6-OHDA model does not seem to reliably counteract the strong local oxidative damage exerted in the striatum after four weeks. While there is a morphologic preservation of dopaminergic cell bodies in the SNpc and a trend to reduced degeneration of axonal terminals in the striatum, these structures largely lack functionality and show loss of intact neurotransmission. This might be due to the strong toxicity of the 6-OHDA model that causes an immediate detrimental effect on dopaminergic fibers in the striatum (Stott \& Barker, 2014) while the downregulation of ROCK2 after AAV injection will be effective after some days. Therefore, a longer observation time in a model with lower lesion severity may have permitted to better observe beneficial effects. Furthermore, an earlier injection time point of the AAV.shRNA constructs may have resulted in a prolonged ROCK2 downregulation and earlier activation of the regenerative response. Nevertheless, this study evaluated the intrinsic role of neuronal ROCK2 as a central regulator for neuronal survival following toxininduced degeneration, while it virtually disregarded potential extrinsic effects generated by microglial and astrocytic responses to neuronal degeneration.

\subsection{The role of pharmacological ROCK inhibition on dynamic presynaptic function}

Since ROCK inhibition is known to facilitate neuronal survival and axonal regeneration in different in vitro and in vivo studies, it is a promising target to improve the treatment of neurodegeneration. However, there is the necessity to fully understand different possible modes of action of a specific ROCK inhibitor before translation to clinical trials. As fasudil is one of the most promising ROCK inhibitors being already successfully employed in the treatment of vasospasms in humans (Shimokawa et al., 2002), we chose this substance for the present investigation.

We thus examined the effect of pharmacological ROCK inhibition on synaptic vesicle dynamics, since the actin cytoskeleton is one of the central structures in mediating synaptic vesicle dynamics and is the main downstream target of ROCK signalling (Dillon \& Goda, 2005; 
Ridley, 2006). Cells were incubated in fluorescently labeled antibody solution and were either electrically stimulated to undergo transmitter release or incubated on ice to generate a negative control. The addition of TTX to the antibody solution of the negative control prohibited the induction of action potentials and therefore minimized the spontaneous bursting activity of the cells (Katz \& Miledi, 1967). Interestingly, the treatment with fasudil for five consecutive days prior to the experiment resulted in a significantly higher fluorescence signal in the unstimulated condition. This means that there was much more antibody binding to the amino terminus of synaptotagmin1 (syt1) in fasudil treated cultures compared to untreated controls. Due to the minimization of vesicle release to the plasma membrane, there should be virtually no vesicle release in the negative controls. Therefore, antibody-labeled structures resulted from surface staining of persistent syt 1 in the outer cell membrane (Brose et al., 1991). It is known, that there are small clusters of remaining syt1 in the plasma membrane of unstimulated cells (Willig et al., 2006) and that synaptic vesicle proteins reside at the synaptic surface for being recaptured during endocytosis (FernándezAlfonso et al., 2006). In our experiments, fasudil treatment therefore seemed to enhance the syt1 clustering on the surface of the plasma membrane observed in unstimulated conditions. This increase in surface syt1 could be caused by three different mechanisms: 1 . Fasudil could enhance the basic spontaneous bursting activity of hippocampal neurons in culture and therefore increase the number in exocytosed vesicles, resulting in more exposure of the intravesicular domain of syt1 to the extracellular space. 2 . There could be fasudil-mediated slower or less endocytosis, which is consequently leading to increased amounts of nonrecycled vesicle proteins on the surface. 3. Long-term fasudil treatment could lead to enhanced expression of the syt1 protein which results in a higher surface pool. To answer this question, we have included the results from the experiments in which the cells were electrically stimulated.

After electrical stimulation the fluorescent signal of syt1 was much brighter than in the unstimulated negative control, indicating that many vesicles underwent the circle of exoand endocytosis (Willig et al., 2006). To investigate potential differences in the amounts of vesicles engaged in transmitter release and vesicle retrieval after fasudil treatment, we compared the fluorescence signals of cultures electrically stimulated with $20 \mathrm{~Hz}$ for $30 \mathrm{~s}$ to the unstimulated surface staining. For better clarification, we calculated $\Delta \mathrm{F}$, which displayed the change in fluorescence signal after stimulation subtracted by the signal of surface 
staining in the respective condition. Interestingly, the fluorescence intensity of fasudil treated cultures had drastically decreased, resulting in a significant smaller $\Delta \mathrm{F}$ compared to untreated cells. This means that after stimulation and intensified vesicle release, fasudil leads to less synaptic vesicles going through the cycle of exocytosis and retrieval, compared to untreated cultures. Moreover, this contradicts the hypothesis of having increased bursting activity in fasudil treated cultures, so this in turn might be not the reason for the enhanced syt1 presence at the surface of cells treated with fasudil.

In order to examine the mechanism responsible for decreased $\Delta \mathrm{F}$, we evaluated the results from the life imaging observations. Given the real-time course of exo- and endocytosis by measuring the changes in fluorescence generated with the $\mathrm{pH}$-sensitive fluorophore CypHer coupled to syt1-antibody, allowed us to evaluate synaptic vesicle kinetics after electrical stimulation. Based on the curves generated for each stimulation event, we could observe similar vesicle kinetics as described before by Fernández-Alfonso and Ryan. They found, that the time of endocytosis during continuous stimulation with $20 \mathrm{~Hz}$ for $30 \mathrm{~s}$ exceeded the time of exocytosis, suggesting a rapid release of the fusion-competent vesicles and a slower recycling phase of these membrane-docked vesicles leading to an accumulation of vesicle compounds on the synaptic surface (Fernández-Alfonso \& Ryan, 2004; Ryan \& Smith, 1995). Calculating the mean time constant $\tau$ as a measure for the speed of either exo- or endocytosis in our study, it revealed no differences between the fasudil treated and untreated cultures. Therefore, in fasudil treated cells the exocytosis of synaptic vesicles appeared to be as fast as in the untreated condition and the same seemed to be true for the vesicle recycling within the 5.5 minutes of observation time. From this point of view, the decreased amounts of incorporated antibody signal after electrical stimulation were not resulting from altered vesicle kinetics. The same would be true for the surface staining of syt1 without stimulation, when it is assumed that the same mechanisms of vesicle internalization are operating the endocytosis of vesicles in the resting state, where basic bursting activity is present, as well as membrane recycling after strong stimulation.

Moreover, changes in the synaptic vesicle release probability are known to be due to the amount of fusion-competent vesicles in the so-called readily-releasable pool (RRP), which represents the small fraction ( $1 \%)$ of vesicles conducting fast exocytosis during physiological activity, while the majority of vesicles remains in the so-called reserve pool (RP) (Rizzoli \& Betz, 2004; Denker et al., 2011). During our stimulation paradigm of $20 \mathrm{~Hz}$ for $30 \mathrm{~s}$ 
we observed a steady decrease in fluorescence until the stimulation stops at $30 \mathrm{~s}$. This indicates continuous exocytosis of synaptic vesicles, which fuse with the plasma membrane as response to strong electrical stimuli. To maintain continuous exocytosis, vesicle release is recruited from vesicles of the so-called recycling pool, which is containing $10-20 \%$ of all vesicles, while the majority of vesicles remains unused in the RP, even during strong stimulation (Rizzoli \& Betz, 2004; Denker et al., 2011). Therefore, the frequent stimuli in our study seemed to induce permanent vesicle recycling, thus, no depletion of the recycle pool was reached after $20 \mathrm{~Hz}$ for $30 \mathrm{~s}$ in fasudil treated and untreated cultures. However, this gives no information about the number of vesicles released simultaneously in the same time, when we assume that the kinetics for exo- and endocytosis are not changed and the retrieval of vesicles from the recycling pool is maintained during stimulation. Regarding our results depicting a decreased $\Delta \mathrm{F}$ in fasudil treated neurons, this suggests a reduction in the total number of vesicles, which were released and recycled induced by electrical stimulation over $30 \mathrm{~s}$. This means that fasudil treatment had reduced the size of the RRP and therefore the amount of vesicles, which underwent the cycle of exo- and endocytosis during particular stimulation periods (Rosenmund et al., 1996). A similar result was found by González-Forero and colleagues in a patch clamp study investigating the electrical activity of rat hypoglossal motoneurons after ROCK inhibition. They observed a significant decrease in postsynaptic input after pharmacological short time (5-10 min prior to the experiment) ROCK inhibition with either Y-27632 or dimethylfasudil (Gonzalez-Forero et al., 2012). They could show that ROCK inhibition leads to a modulation of the presynaptic strength resulting in decreased output caused by reduced transmitter release. Electron microscopy of synaptic boutons revealed a reduction in number of presynaptic vesicles in two areas at the active zone, one directly adjacent to the membrane and the second 0.1-0.2 $\mu \mathrm{m}$ away from the active zone, while there was no significant reduction in the more distant region and in the total vesicle pool size after ROCK inhibition. This suggests a ROCK mediated modulation of the vesicle number in the RRP and the recycling pool. Inhibiting the pathway of ROCK mediated phosphorylation of MLC, which thereby also increases the activity of MLCP, leads to the absence of actin/myosin contractility and non-initiation of stress fiber formation (Raad et al., 2012; Amano et al., 2010). For vesicle exocytosis, the formation of stress fibers is essential to provide cytoskeletal tracks to maintain the physiological guidance of vesicles to the RRP or from the RRP to the active zone (Dillon \& Goda, 2005; Gonzalez-Forero et al., 2012). 
Additionally, during ROCK inhibition and the inactivation of LIMK, cofilin is activated and therefore mediating F-actin depolymerization at the pointed ends, which could interrupt the physiological track to transport vesicles to the membrane (Meyer \& Feldman, 2002). In this case, there would be a shift to more globular actin (G-actin) than filamentous F-actin, which we tested in our study by evaluating the G-/F-actin ratio in hippocampal neurons after longterm ROCK inhibition with fasudil. Unfortunately, there was only one culture available to test this hypothesis, but we could observe a distinct shift to highly elevated G-actin amounts after ROCK inhibition compared to untreated cultures. This gives evidence for the essential role of ROCK activity in maintaining the ultrastructural composition of actin filaments, which therefore enables vesicle recruitment from the reserve pool and the transport to the membrane of the active zone. Of course, the observation of increased G-actin amounts has to be confirmed in further experiments.

Taken together, long-term fasudil treatment seems to decrease the ability of synaptic vesicles to move to the active zone and to fuse to the membrane by interrupting the synaptic actin cytoskeleton, which is normally providing the track for synaptic vesicle movements and is mediated by inactivation of cofilin. However, in our study hippocampal vesicle exo- and endocytosis is not reduced at all after fasudil treatment. Thus, we observed continuous vesicle release and retrieval during strong electrical stimulation, but the size of the RRP and the amount of exo- and endocytosed vesicles seem to have drastically decreased, while the kinetics were not altered. In regard to these findings, the enhanced syt1 amounts observed in the plasma membrane from the unstimulated culture conditions is not due to decreased endocytotic recycling of synaptic vesicle components, but further there might be an upregulation in syt1 expression caused by ROCK inhibition. It is established, that ROCK inhibition by fasudil is changing the transcriptome of astrocytes and alters expression levels of different astrocytic proteins (Lau et al., 2012; Lau et al., 2011). The same is known from inhibition of the ROCK-activator RhoA in the MN9D dopaminergic cell line, where Zhou and colleagues showed evidence for enhanced upregulation of synaptic proteins like VMAT, synaptophysin and synapsin, which was accompanied by a decrease in alpha-synuclein expression (Zhou et al., 2011). Therefore, the expression of synaptic proteins including syt1 might be upregulated by ROCK inhibition even in mature hippocampal synapses, but this has to be confirmed by further investigations, for instance with Western blot analysis or quantitative PCR. 
The role of pharmacological ROCK inhibition in synaptic transmission seems to influence the modulation of a complex network involving the actin/myosin contractility and regulation of actin filaments for vesicle trafficking. However, since fasudil is known to act as a "dirty" drug also affecting several other kinases (Mueller et al., 2005; Davies et al., 2000), other pathways and mechanisms might be involved in vesicle dynamics as well.

Regarding the application of ROCK inhibitors in the treatment of neurodegenerative disorders, the present findings could be of great interest to compensate the lack of dopaminergic transmission in the striatum of PD patients. The loss of dopaminergic inhibition, which is mediated through the postsynaptic D2 receptor in the striatum, results in the disinhibition and overactivation of the subthalamic nucleus (STN), which causes some of the characteristic symptoms of PD (Parent \& Hazrati, 1995; Crittenden \& Graybiel, 2011). Furthermore, output neurons from the STN mediate excitatory innervation of the GPe, GPi, SNpr, pedunculopontine nucleus (PPN) and SNpc via glutamatergic transmission (Futami et al., 1995). Already damaged nigral dopaminergic neurons are thereby exposed to an extensive glutamatergic innervation through postsynaptic $\mathrm{N}$-methyl-D-aspertate (NMDA) receptors that could cause excitotoxic damage (Olanow \& Tatton, 1999; Rodriguez et al., 1998, Figure 4.1-A). This may induce excessive intracellular calcium influx, which activates nitric oxide synthesis, generation of free radicals and thereby activates apoptotic mechanisms (Dong et al., 2009). As we observed in our in vitro study a significant reduction of the vesicle release after ROCK inhibition with fasudil from hippocampal glutamatergic and GABAergic neurons, we suggest that fasudil mediated ROCK inhibition could also play an essential role to decrease glutamate release from STN terminals at nigral dopaminergic postsynapses in PD (Figure 4.1-B). However, the modulation of synaptic RRP by ROCK inhibition might affect all excitatory and inhibitory synaptic structures. Therefore, application of ROCK inhibitors to modify specific transmitter release has to be further investigated for different types of synapses in different cell types of the mature brain. 


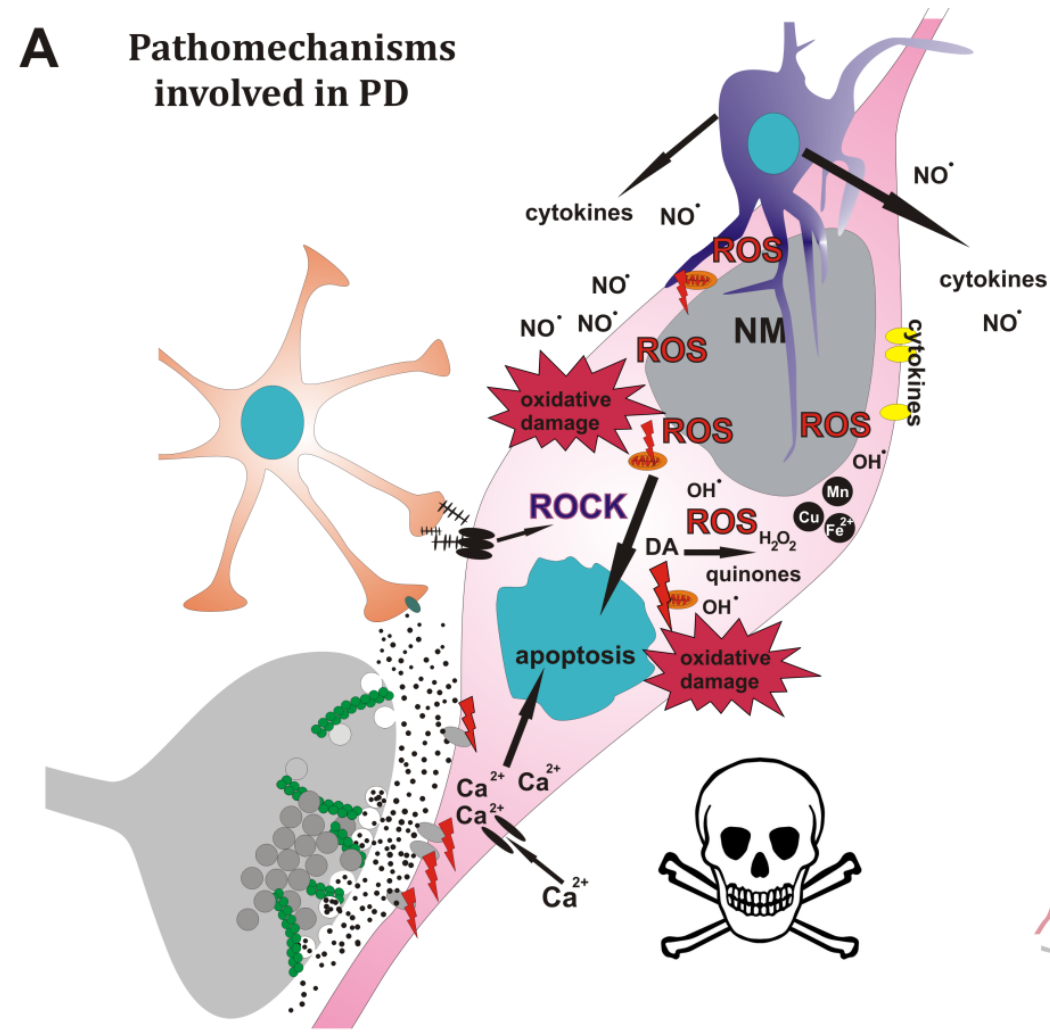

\section{B Attenuation of pathology by ROCK inhibition}

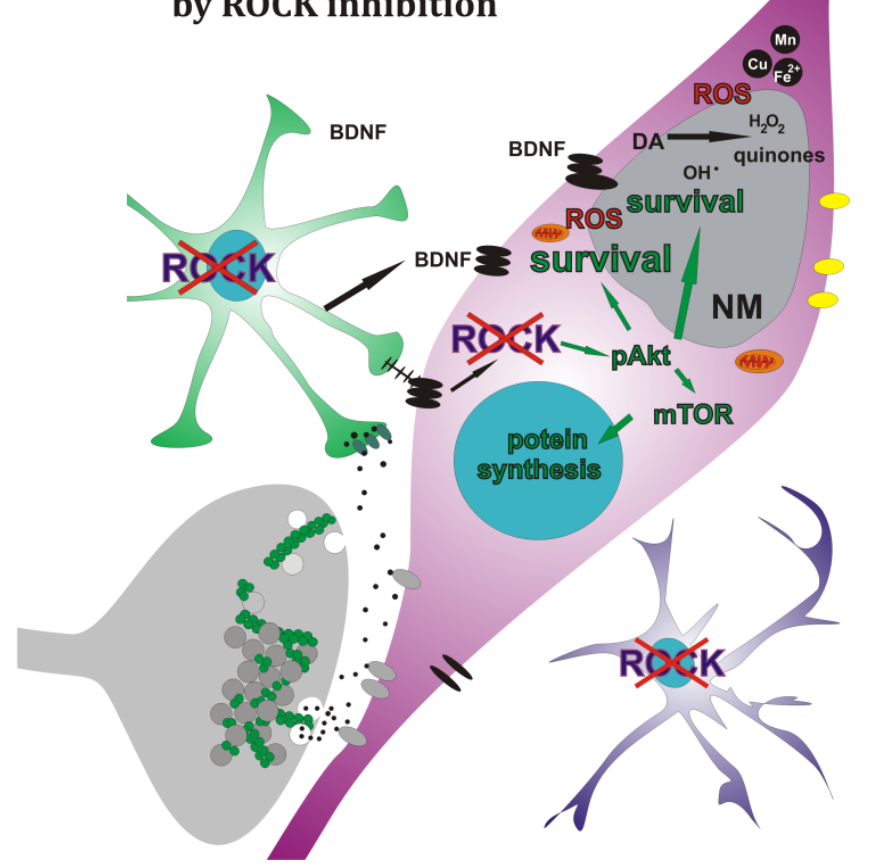

reactive microglia reactive astrocyte

DAergic neuron

glutamatergic presynapse

Nogo/LINGO/p75

receptor complex

HW CSPG

cytokin receptor

actin filament

NMDA

EAAT

- synaptic vesicle

$\because$ glutamate

- Ca channel

30 astrocyte

2 microglia

Figure 4.1: Illustration of pathomechanisms in PD progression and their possible attenuation by pharmacological ROCK inhibition. Reactive astro- and microglia damage the dopaminergic neuron via glutamate excitotoxicity and finally trigger apoptosis (A). Under pharmacological ROCK inhibition, astrocytes could generate a protective phenotype, while microglia could be inhibited in cytoskeleton reorganisation and migration (B). Excitotoxicity could be prevented by reduced vesicle trafficking to the active zone in glutamatergic presynapses and intraneuronal survival pathways could be enhanced by intrinsic neuronal ROCK 
inhibition. $\mathrm{NM}=$ neuromelanin, $\mathrm{DA}=$ dopamine, $\mathrm{NO} \bullet=$ nitric oxide, $\mathrm{OH} \bullet=$ hydroxyl radical, $\mathrm{H}_{2} \mathrm{O}_{2}=$ hydrogen peroxide, $\mathrm{Ca}=$ calcium, $\mathrm{Mn}=$ manganese, $\mathrm{Fe}=$ iron.

\subsection{Conclusion}

All three experiments illustrate the importance of the Rho kinase pathway in the treatment of PD. The decreasing regeneration capacity of neurons during aging together with additive not yet clarified pathological mechanisms lead to the characteristic neuronal loss in the nigrostriatal system. Nevertheless, there is a growing body of evidence for the employment of ROCK inhibitors as promising treatment for PD and we could show that at least in striatal astroglial cells the ROCK expression is altered in PD cases. Additionally, our investigations about the specificity of neuronal ROCK2 downregulation via shRNA and the potential role in transmitter release as well as the knowledge about ROCK inhibition from previous studies in neurodegeneration and glial modulation, support the application of ROCK inhibitors also in clinical trials for the treatment of PD (Figure 4.1-A+B). 


\section{Summary}

The cure of neurodegenerative diseases is one of the main goals in today's neuroscience research. One of the main obstacles is the delayed diagnostics in relation to molecular pathology. In Parkinson's disease (PD), several pathophysiological mechanisms participating in neurodegeneration have been elucidated, e.g. protein aggregation, increased oxidative stress, mitochondrial malfunction etc., but the precise etiological cascade still remains elusive (Dauer \& Przedborski, 2003; Dickson et al., 2009). In order to prevent the progression of neuronal degeneration and to facilitate regeneration the potential of modulating the Rho kinase (ROCK) pathway is gaining recognition.

Here we could show that there seems to be an age-dependent decrease in the expression of the growth associated protein GAP-43, accompanied by astroglia infiltration and declined synaptophysin abundance in certain brain areas, which are affected in PD. These age-related changes are exacerbated in post mortem PD brains, but it is still unknown whether this is the cause or the consequence of neurodegeneration. Furthermore, ROCK protein expression seems to be altered at least in striatal astrocytes, indicating a role of the ROCK signaling cascade in neurodegeneration. The findings of attenuated dopaminergic cell loss after specific shRNA mediated ROCK2 downregulation in neurons of the substantia nigra $(\mathrm{SN})$ in the 6-OHDA mouse model of PD, showed the potential of intrinsic ROCK2 inhibition to promote neuronal survival. Moreover, we here evaluated the role of ROCK inhibition in the context of transmitter regulation. We could show that pharmacological long-term ROCK inhibition is attenuating transmitter release by reducing the readily releasable pool (RRP) of synaptic vesicles in vitro. Thus, additive detrimental effects generated by excitotoxicity from overactive glutamatergic synapses in the progression of PD, could potentially be antagonized by ROCK inhibition in the respective cells. Nevertheless, further investigations are required to evaluate other functions of ROCK inhibition in regard to the modulation of cytoskeletal rearrangements and the effects on other transmitter systems.

Taken together, by affecting cell survival, neuronal growth, glial responses and transmitter release, the modulation of the ROCK pathway is a highly promising target for the future treatment of PD and other neurodegenerative disorders and warrants more in-depth pre-clinical and clinical evaluation. 


\section{References}

Alvarez-Fischer, D. et al., 2008. Characterization of the striatal 6-OHDA model of Parkinson's disease in wild type and alpha-synuclein-deleted mice. Experimental neurology, 210(1), pp.182-93.

Amano, M., Nakayama, M. \& Kaibuchi, K., 2010. Rho-kinase/ROCK: A key regulator of the cytoskeleton and cell polarity. Cytoskeleton (Hoboken, N.J.), 67(9), pp.545-54.

Aron, L. \& Klein, R., 2011. Repairing the parkinsonian brain with neurotrophic factors. Trends in neurosciences, 34(2), pp.88-100.

Arpin, M. et al., 2014. Emerging role for ERM proteins in cell adhesion and migration. Cell Adhesion \& Migration, 5(2), pp.199-206.

Barcia, C. et al., 2012. ROCK/Cdc42-mediated microglial motility and gliapse formation lead to phagocytosis of degenerating dopaminergic neurons in vivo. Scientific reports, 2 , p.809.

Bartlett, J.S., Samulski, R.J. \& McCown, T.J., 1998. Selective and rapid uptake of adenoassociated virus type 2 in brain. Human gene therapy, 9(8), pp.1181-6.

Bergman, H. \& Deuschl, G., 2002. Pathophysiology of Parkinson's disease: from clinical neurology to basic neuroscience and back. Journal of the Movement Disorder Society, 17 Suppl 3, pp.S28-40.

Bermel, C. et al., 2009. Combined inhibition of Cdk5 and ROCK additively increase cell survival, but not the regenerative response in regenerating retinal ganglion cells. Molecular and cellular neurosciences, 42(4), pp.427-37.

Bernheimer, H. et al., 1973. Brain Dopamine and the Syndromes of Parkinson and Huntington. Journal of Neurological Science. 4, pp.145-148.

Blesa, J. et al., 2012. Classic and new animal models of Parkinson's disease. Journal of biomedicine \& biotechnology, 2012, p.845618.

Blits, B. et al., 2010. Adeno-associated viral vector (AAV)-mediated gene transfer in the red nucleus of the adult rat brain: comparative analysis of the transduction properties of seven AAV serotypes and lentiviral vectors. Journal of neuroscience methods, 185(2), pp.257-63.

Blum, D. et al., 2001. Molecular pathways involved in the neurotoxicity of 6-OHDA, dopamine and MPTP: contribution to the apoptotic theory in Parkinson' s disease. Progress in Neurobiology, 65, pp.135-172. 
Braak, H. et al., 2003. Idiopathic Parkinson's disease: possible routes by which vulnerable neuronal types may be subject to neuroinvasion by an unknown pathogen. Journal of neural transmission, 110(5), pp.517-36.

Braak, H. et al., 2003. Staging of brain pathology related to sporadic Parkinson's disease. Neurobiology of Aging, 24(2), pp.197-211.

Braak, H. \& Braak, E., 1991. Neuropathological stageing of Alzheimer-related changes. Acta Neuropathologica. pp.239-259.

Brabeck, C. et al., 2004. Lesional expression of RhoA and RhoB following traumatic brain injury in humans. Journal of neurotrauma, 21(6), pp.697-706.

Brodin, L., Löw, P. \& Shupliakov, O., 2000. Sequential steps in clathrin-mediated synaptic vesicle endocytosis. Current Opinion in Neurobiology, 10(3), pp.312-320.

Brose, N. et al., 1991. Synaptotagmin: A Calcium Sensor on the Synaptic Vesicle Surface. Science, 265.1021-1025.

Bucciantini, M. et al., 2002. Inherent toxicity of aggregates implies a common mechanism for protein misfolding diseases. Nature, 416(6880), pp.507-11.

Burke, R.E. \& O'Malley, K., 2013. Axon degeneration in Parkinson's disease. Experimental neurology, 246, pp.72-83.

Burke, S.N. \& Barnes, C. a, 2006. Neural plasticity in the ageing brain. Nature reviews. Neuroscience, 7(1), pp.30-40.

Cafferty, W.B.J. et al., 2010. MAG and OMgp synergize with Nogo-A to restrict axonal growth and neurological recovery after spinal cord trauma. The Journal of Neuroscience, 30(20), pp.6825-37.

Cannon, J.R. \& Greenamyre, J.T., 2010. Neurotoxic in vivo models of Parkinson's disease recent advances. Progress in brain research, 184, pp.17-33.

Chadi, G. et al., 1993. Protective actions of human recombinant basic fibroblast growth factor on MPTP-lesioned nigrostriatal dopamine neurons after intraventricular infusion. Exp. Brain Research, pp.145-158.

Chandra, S. et al., 2003. A broken alpha -helix in folded alpha -Synuclein. The Journal of biological chemistry, 278(17), pp.15313-8.

Chartier-Harlin, M.-C. et al., 2004. Alpha -synuclein locus duplication as a cause of familial Parkinson's disease. Lancet, 364 pp.1167-1169.

Cheng, J.C., Moore, T.B. \& Sakamoto, K.M., 2003. RNA interference and human disease. Molecular Genetics and Metabolism, 80(1-2), pp.121-128. 
Choi, B.-K. et al., 2013. Large $\alpha$-synuclein oligomers inhibit neuronal SNARE-mediated vesicle docking. Proceedings of the National Academy of Sciences of the United States of America, 110(10), pp.4087-92.

Clarkson, E.D., Zawada, W.M. \& Freed, C.R., 1997. GDNF improves survival and reduces apoptosis in human embryonic dopaminergic neurons in vitro. Cell and Tissue Research, 289(2), pp.207-210.

Cohen, P., 2002. Protein kinases - the major drug targets of the twenty-first century? Nature publishing group 309-315.

Crittenden, J.R. \& Graybiel, A.M., 2011. Basal Ganglia disorders associated with imbalances in the striatal striosome and matrix compartments. Frontiers in neuroanatomy, 5(September), p.59.

Damier, P. et al., 1993. Glutathione peroxidase, glial cells and Parkinson's disease. Neuroscience, 52(1), pp.1-6.

Daniel, J. a et al., 2012. Analysis of synaptic vesicle endocytosis in synaptosomes by highcontent screening. Nature Protocols, 7(8), pp.1439-1455.

Dauer, W. \& Przedborski, S., 2003. Parkinson's Disease: Mechanisms and Models. Neuron, 39, pp.889-909.

Davies, S.P. et al., 2000. Specificity and mechanism of action of some commonly used protein kinase inhibitors. Biochem, 105, pp.95-105.

Dawson, T.M. \& Dawson, V.L., 2003. Molecular pathways of neurodegeneration in Parkinson's disease. Science (New York, N.Y.), 302(5646), pp.819-22.

DeLau, L.M.L. \& Breteler, M.M.B., 2006. Epidemiology of Parkinson's disease. Lancet, 5, pp.525-535.

DeLong, M. \& Wichmann, T., 2007. Circuits and Circuit Disorders of the Basal Ganglia. Neurological review, 64. 20-24.

Denker, A. et al., 2011. From the Cover: A small pool of vesicles maintains synaptic activity in vivo. Proceedings of the National Academy of Sciences, 108(41), pp.17177-17182.

Denker, A. et al., 2011. The reserve pool of synaptic vesicles acts as a buffer for proteins involved in synaptic vesicle recycling. Proceedings of the National Academy of Sciences, 108(41), pp.17183-17188.

Deuschl, G. \& Bergman, H., 2002. Pathophysiology of nonparkinsonian tremors. Movement disorders. 17 Suppl 3, pp.S41-8.

Dexter, D.T. \& Jenner, P., 2013. Parkinson disease: from pathology to molecular disease mechanisms. Free radical biology \& medicine, 62, pp.132-44. 
Dickson, D.W. et al., 2009. Neuropathological assessment of Parkinson's disease: refining the diagnostic criteria. The Lancet. Neurology, 8(12), pp.1150-7.

Dillon, C. \& Goda, Y., 2005. The actin cytoskeleton: integrating form and function at the synapse. Annual review of neuroscience, 28, pp.25-55.

Dong, X., Wang, Y. \& Qin, Z., 2009. Molecular mechanisms of excitotoxicity and their relevance to pathogenesis of neurodegenerative diseases. Acta pharmacologica Sinica, 30(4), pp.379-87.

Dudek, H. et al., 1997. Regulation of Neuronal Survival by the Serine-Threonine Protein Kinase Akt. Science. 275, pp.661-665.

Dudek, S.M. \& Garcia, J.G.N., 2003. Rho family of guanine exchange factors (GEFs) in cellular activation: who's dancing? And with whom? Circulation research, 93(9), pp.794-5.

Dunnett, S.B. \& Björklund, A., 1999. Prospects for new restorative and neuroprotective treatments in Parkinson's disease. Nature, 399.A32-A39.

During, M.J. \& Leone, P., 1995. Adeno-associated virus vectors for gene therapy of neurodegenerative disorders. Clinical neuroscience, 3(5), pp.292-300.

Van Dyck, C. et al., 2002. Age related decline in Dopamine Transporters. Analysis of Striatal Subregions, Nonlinear Effects and hemispheric asymmetries. Geriatr. Psychiatry, 10:1. pp.36-43.

Eichholtz, T. et al., 1993. The bioactive phospholipid lysophosphatidic acid is released from activated platelets. Biochem, 291, pp.677-680.

Fawcett, J.W., 1992. Intrinsic neuronal determinants of regeneration. Trends in Neurosciences, 15(1), pp.5-8.

Fearnley, J.M. \& Lees, A.J., 1991. Ageing and Parkinson's disease: substantia nigra regional selectivity. Brain, pp.2283-2301.

Federoff, H.J. et al., 2003. Parkinson's disease. The life of the dopaminergic neuron. Annals of the New York academy of Science. Vol 991.

Fernández-Alfonso, T., Kwan, R. \& Ryan, T. A., 2006. Synaptic vesicles interchange their membrane proteins with a large surface reservoir during recycling. Neuron, 51(2), pp.179-86.

Fernández-Alfonso, T. \& Ryan, T. A., 2004. The Kinetics of Synaptic Vesicle Pool Depletion at CNS Synaptic Terminals. Neuron, 41(6), pp.943-953.

Finkelstein, D.I. et al., 2000. Axonal sprouting following lesions of the rat substantia nigra. Neuroscience, 97(1), pp.99-112. 
Flotte, T.R. \& Carter, B.J., 1995. Adeno-associated virus vectors for gene therapy. Gene therapy, 2(6), pp.357-62.

Forman, M.S., Trojanowski, J.Q. \& Lee, V.M., 2004. OUR TENTH YEAR Neurodegenerative diseases: a decade of discoveries paves the way for therapeutic breakthroughs. Nature medicine, 10(10), pp.1055-1063.

Frey, D. et al., 2000. Shared and Unique Roles of CAP23 and GAP43 in Actin Regulation, Neurite Outgrowth, and Anatomical Plasticity. Cell Biology, 149(7), pp.1443-1453.

Futami, T., Takakusaki, K. \& Kitai, S.T., 1995. Glutamatergic and cholinergic inputs from the pedunculopontine tegmental nucleus to dopamine neurons in the substantia nigra pars compacta. Neuroscience Research, 21(4), pp.331-342.

Fyrst, H. \& Saba, J.D., 2011. An update on sphingosine-1-phosphate and other sphingolipid mediators. Nat Chem Biol, 6(7), pp.489-497.

Gallo, G. \& Letourneau, P.C., 2004. Regulation of growth cone actin filaments by guidance cues. Journal of neurobiology, 58(1), pp.92-102.

Gehler, S. et al., 2004. p75 neurotrophin receptor signaling regulates growth cone filopodial dynamics through modulating RhoA activity. The Journal of neuroscience, 24(18), pp.4363-72.

Georgievska, B., 2002. Aberrant Sprouting and Downregulation of Tyrosine Hydroxylase in Lesioned Nigrostriatal Dopamine Neurons Induced by Long-Lasting Overexpression of Glial Cell Line Derived Neurotrophic Factor in the Striatum by Lentiviral Gene Transfer. Experimental Neurology, 177(2), pp.461-474.

Gibb, W.R.G. \& Lees, A.J., 1988. Occasional review. The relevance of the Lewy body to the pathogenesis of idiopathic Parkinson's disease. Journal of Neurology, Neurosurgery and Psychiatry, pp.745-752.

Goetzl, E.J., 2007. Diverse pathways for nuclear signaling by G protein-coupled receptors and their ligands. official publication of the Federation of American Societies for Experimental Biology, 21(3), pp.638-42.

Goldberg, J.L. et al., 2004. An oligodendrocyte lineage-specific semaphorin, Sema5A, inhibits axon growth by retinal ganglion cells. The Journal of neuroscience, 24(21), pp.4989-99.

Goldshmit, Y., McLenachan, S. \& Turnley, A., 2006. Roles of Eph receptors and ephrins in the normal and damaged adult CNS. Brain research reviews, 52(2), pp.327-45.

Gonzalez-Forero, D. et al., 2012. Endogenous Rho-Kinase Signaling Maintains Synaptic Strength by Stabilizing the Size of the Readily Releasable Pool of Synaptic Vesicles. Journal of Neuroscience, 32(1), pp.68-84. 
Graham, D.G., 1978. Oxidative pathways for catecholamines in the genesis of neuromelanin and cytotoxic quinones. Molecular pharmacology, 14. pp.633-643.

Günther, R. et al., 2014. The rho kinase inhibitor Y-27632 improves motor performance in male SOD1(G93A) mice. Frontiers in neuroscience, 8, p.304.

Guertin, D. A. \& Sabatini, D.M., 2007. Defining the role of mTOR in cancer. Cancer cell, 12(1), pp.9-22.

Hagg, T. \& Oudega, M., 2006. Degenerative and Spontaneous Regenerative Processes. Jrnl. of Neurotrauma, 23(3), pp.264-280.

Hanisch, U.-K. \& Kettenmann, H., 2007. Microglia: active sensor and versatile effector cells in the normal and pathologic brain. Nature neuroscience, 10(11), pp.1387-94.

Hashimoto, R. et al., 1999. Distribution of Rho-kinase in the bovine brain. Biochemical and biophysical research communications, 263(2), pp.575-9.

Henkel, J.S. et al., 2009. Microglia in ALS: the good, the bad, and the resting. Journal of neuroimmune pharmacology, 4(4), pp.389-98.

Hidalgo-Figueroa, M. et al., 2012. GDNF Is Predominantly Expressed in the Neostriatal Interneuronal Ensemble in Normal Mouse and after Injury of the Nigrostriatal Pathway. Journal of Neurosciences, 32(3), pp.864-872.

Hirsch, E.C. \& Hunot, S., 2009. Neuroinflammation in Parkinson's disease: a target for neuroprotection. Lancet, 8. 382-397.

Hornykiewicz, O., 2001. Chemical neuroanatomy of the basal ganglia - normal and in Parkinson's disease. Journal of Chemical Neuroanatomy, 22(1-2), pp.3-12.

Hsieh, T. et al., 2011. Time-course gait analysis of hemiparkinsonian rats following 6hydroxydopamine lesion. Behavioural Brain Research, 222(1), pp.1-9.

Hunter, R.L. et al., 2007. Inflammation induces mitochondrial dysfunction and dopaminergic neurodegeneration in the nigrostriatal system. Journal of neurochemistry, 100(5), pp.1375-86.

Hutchins, J.B. \& Barger, S.W., 1998. Why neurons die: cell death in the nervous system. The Anatomical record, 253(3), pp.79-90.

Ishizaki, T. et al., 1996. The small GTP-binding protein Rho binds to and activates a $160 \mathrm{kDa}$ Ser/Thr protein kinase homologous to myotonic dystrophy kinase. Molecular Pharmacology, 15(8), pp.1885-1893.

Iwata, S.-I., Nomoto, M. \& Fukuda, T., 2001. Regulation of GAP-43 Protein and mRNA in Nigrostriatal Dopaminergic Neurons After the Partial Destruction of Dopaminergic Terminals With Intrastriatal 6-Hydroxydopamine. Synapse, 22, pp.16-22. 
Kandel, E.R. et al., 2000. Principles of Neuroscience. 4th Edition. The McGraw-Hill Companies. New York, USA.

Katz, B.Y.B. \& Miledi, R., 1967. A study of synaptic transmission in the absence of nerve impulses. J. Physiology, 192. pp.407-436.

Kiely, A.P. et al., 2013. $\alpha$-Synucleinopathy associated with G51D SNCA mutation: a link between Parkinson's disease and multiple system atrophy? Acta neuropathologica, 125(5), pp.753-69.

Kim, S.R. et al., 2011. Dopaminergic Pathway Reconstruction by Akt / Rheb-Induced Axon Regeneration. American Neurological Association, pp.1-11.

Kim, Y.S. \& Joh, T.H., 2006. Microglia, major player in the brain inflammation: their roles in the pathogenesis of Parkinson's disease. Experimental \& molecular medicine, 38(4), pp.333-47.

Kirik, D., Georgievska, B. \& Björklund, A., 2004. Localized striatal delivery of GDNF as a treatment for Parkinson disease. Nature Neurosciences, 7(2), pp.105-110.

Klein, R.L. et al., 1999. Generation of aberrant sprouting in the adult rat brain by GAP-43 somatic gene transfer. Brain Research, 832(1-2), pp.136-144.

Koch, J.C. et al., 2011. Imaging of rat optic nerve axons in vivo. Nature protocols, 6(12), pp.1887-96.

Koch, J.C. et al., 2014. ROCK2 is a major regulator of axonal degeneration, neuronal death and axonal regeneration in the CNS. Cell death \& disease, 5, p.e1225.

Komagome, R., Kimura, K. \& Saito, M., 2000. Postnatal changes in Rho and Rho-related proteins in the mouse brain. Jpn.J Vet. Res. 47(3-4):127-133

Kottis, V. et al., 2002. Oligodendrocyte-myelin glycoprotein (OMgp) is an inhibitor of neurite outgrowth. Journal of Neurochemistry, 82(6), pp.1566-1569.

Lashuel, H.A. et al., 2013. The many faces of $\alpha$-synuclein: from structure and toxicity to therapeutic target. Nature reviews. Neuroscience, 14(1), pp.38-48.

Lau, C.L. et al., 2011. The Rho kinase inhibitor Fasudil up-regulates astrocytic glutamate transport subsequent to actin remodelling in murine cultured astrocytes. British journal of pharmacology, 163(3), pp.533-45.

Lau, C.L. et al., 2012. Transcriptomic profiling of astrocytes treated with the Rho kinase inhibitor fasudil reveals cytoskeletal and pro-survival responses. Journal of cellular physiology, 227(3), pp.1199-211.

Laux, T. et al., 2000. GAP-43, MARCKS, and CAP23 modulate PI(4,5)P2 at plasmalemmal Rafts, and Regulate Cell Cortex Actin Dynamics through a Common Mechanism. Cell Biology, 149(7), pp.1455-1471. 
Lázaro, D.F. et al., 2014. Systematic Comparison of the Effects of Alpha-synuclein Mutations on Its Oligomerization and Aggregation. PLoS genetics, 10(11), p.e1004741.

Li, Z. et al., 2005. Regulation of PTEN by Rho small GTPases. Nature cell biology, 7(4), pp.399-404.

Lingor, P. et al., 2007. Inhibition of Rho kinase (ROCK) increases neurite outgrowth on chondroitin sulphate proteoglycan in vitro and axonal regeneration in the adult optic nerve in vivo. Journal of neurochemistry, 103(1), pp.181-9.

Lingor, P. et al., 2008. ROCK inhibition and CNTF interact on intrinsic signalling pathways and differentially regulate survival and regeneration in retinal ganglion cells. Brain, $131(\mathrm{Pt}$ 1), pp.250-63.

Love, 2005. Glial cell line - derived neurotrophic factor induces neuronal sprouting in human brain. Nature Medicine, 11(7), pp.703-704.

Löw, K. \& Aebischer, P., 2012. Use of viral vectors to create animal models for Parkinson's disease. Neurobiology of disease, 48(2), pp.189-201.

Maekawa, M., 1999. Signaling from Rho to the Actin Cytoskeleton Through Protein Kinases ROCK and LIM-kinase. Science, 285(5429), pp.895-898.

Maragakis, N.J. \& Rothstein, J.D., 2006. Mechanisms of Disease: astrocytes in neurodegenerative disease. Nature clinical practice, 2(12), pp.679-89.

Martin, L.J., 2001. Neuronal cell death in nervous system development, disease, and injury. Journal of Molecular Medicine. pp455-478.

McCarroll, J.A. et al., 2014. TUBB3/BIII-tubulin acts through the PTEN/AKT signaling axis to promote tumorigenesis and anoikis resistance in non-small cell lung cancer. Cancer research.

McCown, T.J. et al., 1996. Differential and persistent expression patterns of CNS gene transfer by an adeno-associated virus (AAV) vector. Brain Research, 713(1-2), pp.99107.

McGeer, P.L. \& McGeer, E.G., 2008. Glial reactions in Parkinson's disease. Movement disorders, 23(4), pp.474-83.

McKeith, I.G. et al., 2005. Diagnosis and management of dementia with Lewy bodies. Third report of the DLB consortium. Neurology, pp.1-10.

Meier, F.E. et al., 2009. Effect of the farnesyl transferase inhibitor lonafarnib on sensitivity of melanoma cells to the multikinase inhibitor sorafenib and on Rheb farnesylation and mTOR signaling. ASCO Meeting Abstracts, 27(15S), p.9077.

Meyer, G. \& Feldman, E.L., 2002. Signaling mechanisms that regulate actin-based motility processes in the nervous system. Journal of Neurochemistry, 83(3), pp.490-503. 
Mi, S. et al., 2004. LINGO-1 is a component of the Nogo-66 receptor/p75 signaling complex. Nature neuroscience, 7(3), pp.221-8.

Miller, G.W. et al., 1999. Immunochemical analysis of vesicular monoamine transporter (VMAT2) protein in Parkinson's disease. Experimental neurology, 156(1), pp.138-48.

Mirza, B. et al., 2000. The absence of reactive astrocytosis is indicative of a unique inflammatory process in parkinson's disease. Neuroscience, 95(2), pp.425-432.

Mitsumoto, Y. et al., 1998. Spontaneous regeneration of nigrostriatal dopaminergic neurons in MPTP-treated C57BL/6 mice. Biochemical and biophysical research communications, 248(3), pp.660-3.

Monnier, P.P. et al., 2003. The Rho/ROCK pathway mediates neurite growth-inhibitory activity associated with the chondroitin sulfate proteoglycans of the CNS glial scar. Molecular and Cellular Neuroscience, 22(3), pp.319-330.

Morales, M., Colicos, M.A. \& Goda, Y., 2000. Actin-Dependent Regulation of Neurotransmitter Release at Central Synapses. Neuron, 27(3), pp.539-550.

Morrison, J.H. \& Hof, P.R., 1997. Life and Death of Neurons in the Aging Brain. Science, 278. 412-419.

Mueller, B.K., Mack, H. \& Teusch, N., 2005. Rho kinase, a promising drug target for neurological disorders. Nature reviews, 4(5), pp.387-98.

Nakajima, K. \& Kohsaka, S., 2001. Microglia: Activation and Their Significance in the Central Nervous System. Journal of Biochemistry, 130(2), pp.169-175.

Neve, R.L. et al., 1988. Growth-associated protein GAP-43 is expressed selectively in associative regions of the adult human brain. Proc. Natl. acad. Sci, 85(May), pp.36383642.

Obeso, J. a et al., 2010. Missing pieces in the Parkinson's disease puzzle. Nature medicine, 16(6), pp.653-61.

Obeso, J.A. et al., 2002. The Basal Ganglia and Disorders of Movement: Pathophysiological Mechanisms. News Physiol Sci., pp.51-55.

Okamura, N. et al., 2010. In vivo measurement of vesicular monoamine transporter type 2 density in Parkinson disease with ${ }^{18} \mathrm{~F}-\mathrm{AV}-133$. Journal of nuclear medicine, 51(2), pp.223-8.

Olanow, C.W. \& Tatton, W.G., 1999. Etiology and pathogenesis of Parkinson's disease. Annual review of neuroscience, 22, pp.123-44.

Ouchi, Y. et al., 2005. Microglial activation and dopamine terminal loss in early Parkinson's disease. Annals of neurology, 57(2), pp.168-75. 
Paddison, P.J. et al., 2002. Short hairpin RNAs (shRNAs) induce sequence-specific silencing in mammalian cells. Genes \& development, 16(8), pp.948-58.

Pakkenberg, A.K.S.B., 2004. Histological changes of the dopaminergic nigrostriatal system in aging. Cell tissue Res., pp.81-92.

Parent, A. \& Hazrati, L., 1995. Functional anatomy of the basal ganglia. 1. The cortico-basal ganglia-thalamo-cortical loop. Brain Research Reviews, 20, pp.91-127.

Park, J. et al., 2011. Rho-associated kinase connects a cell cycle-controlling anchorage signal to the mammalian target of rapamycin pathway. The Journal of biological chemistry, 286(26), pp.23132-41.

Parkinson, J., 1817. An Essay on the Shaking Palsy. Whittingham and Roland. Reprint 2007. London, UK

Parkkinen, L. et al., 2011. Disentangling the relationship between lewy bodies and nigral neuronal loss in Parkinson's disease. Journal of Parkinson's disease, 1(3), pp.277-86.

Paxinos, G. \& Watson, C., 2001.The mouse brain atlas. $3^{\text {rd }}$ Edition. Elsevier.

Pereira, J.B. et al., 2012. Assessment of cortical degeneration in patients with Parkinson's disease by voxel-based morphometry, cortical folding, and cortical thickness. Human brain mapping, 33(11), pp.2521-34.

Peterson, W.M. et al., 2000. Ciliary Neurotrophic Factor and Stress Stimuli Activate the JakSTAT Pathway in Retinal Neurons and Glia. Neurosciences, 20(11), pp.4081-4090.

Pillon, B. et al., 1989. Does cognitive impairment in Parkinson' s disease result from nondopaminergic lesions? Journal of Neurology, Neurosurgery and Psychiatry, pp.201-206.

Pimashkin, A. et al., 2011. Spiking signatures of spontaneous activity bursts in hippocampal cultures. Frontiers in computational neuroscience, 5, p.46.

Porter, A.G. \& Jänicke, R.U., 1999. Emerging roles of caspase-3 in apoptosis. Cell death and Differentiation, pp.99-104.

Proukakis, C. et al., 2013. A novel alpha-synuclein missense mutation in Parkinson's disease. Neurology, pp.1062-1064.

Prusiner, S.B., 2001. SHATTUCK LECTURE - Neurodegenerative diseases and prions. New England Journal of Medicine, 344(20), pp.1516-1526.

Purves, D. et al., 2004. Neuroscience, 3rd Edition, Sinquer Associates Inc., Massachusetts, USA

Raad, M. et al., 2012. Neuroproteomics approach and neurosystems biology analysis: ROCK inhibitors as promising therapeutic targets in neurodegeneration and neurotrauma. Electrophoresis, 33(24), pp.3659-68. 
Rêdowicz, M.J., 2002. Myosins and pathology: genetics and biology. Acta Biochemica Polonica, 49(4), pp.789-804.

Reynolds, A. et al., 2004. Rational siRNA design for RNA interference. Nature biotechnology, 22(3), pp.326-30.

Ridley, A.J., 2006. Rho GTPases and actin dynamics in membrane protrusions and vesicle trafficking. Trends in cell biology, 16(10), pp.522-9.

Rizzoli, S.O. \& Betz, W.J., 2004. The structural organization of the readily releasable pool of synaptic vesicles. Science, 303(5666), pp.2037-9.

Rochet, J. et al., 2004. Interactions Among $\alpha$-Synuclein, Dopamine, and Biomembranes. Journal of Molecular Neurosciences, 23, pp.23-33.

Rodriguez, M.C., Obeso, J. a. \& Olanow, C.W., 1998. Subthalamic nucleus-mediated excitotoxicity in Parkinson's disease: A target for neuroprotection. Ann. Neurology, 44: 174-188.

Rosenmund, C., Stevens, C.F. \& Zellula, A.G., 1996. Definition of the Readily Releasable Pool of Vesicles at Hippocampal Synapses. Neuron, 16, pp.1197-1207.

Ross, C. a \& Poirier, M. a, 2004. Protein aggregation and neurodegenerative disease. Nature medicine, 10, pp.S10-17.

Ryan, T. a \& Smith, S.J., 1995. Vesicle pool mobilization during action potential firing at hippocampal synapses. Neuron, 14(5), pp.983-989.

Saal, K.-A. et al., 2015. AAV.shRNA-mediated downregulation of ROCK2 attenuates degeneration of dopaminergic neurons in toxin-induced models of Parkinson's disease in vitro and in vivo. Neurobiology of disease, 73, pp.150-62.

Sakaba, T. \& Neher, E., 2003. Involvement of Actin Polymerization in Vesicle Recruitment at the Calyx of Held Synapse. Journal of Neurosciences, 23(3), pp.837-846.

Schmidt, J.T., 2004. Activity-driven sharpening of the retinotectal projection: the search for retrograde synaptic signaling pathways. Journal of neurobiology, 59(1), pp.114-33.

Schwarting, R.K.W. \& Huston, J.P., 1996. The unilateral 6-hydroxydopamine lesion model in behavioral brain research. Analysis olf functional deficits, recovery and treatments. Progress in Neurobiology, 50. 275-331.

Seasholtz, T.M., Majumdar, M. \& Brown, J.H., 1999. Rho as a Mediator of G Protein-Coupled Receptor Signaling. Molecular Pharmacology, 956, pp.949-956.

Seibyl, J.P. et al., 1995. Decreased Single-Photon Emission Computed Tomographc $\{1231$ ) pCIT Striatal Uptake Correlates with Symptom Severity in Parkinson's Disease. American Neurological Association, pp.589-598. 
Shevtsova, Z. et al., 2005. Promoters and serotypes: targeting of adeno-associated virus vectors for gene transfer in the rat central nervous system in vitro and in vivo. Experimental physiology, 90(1), pp.53-9.

Shi, J. \& Wei, L., 2008. Rho kinase in the regulation of cell death and survival. Arch Immunol. Ther Exp., 55(2), pp.61-75.

Shibuya, M. et al., 1992. Effect of AT877 on cerebral vasospasm after aneurysmal subarachnoid hemorrhage. J. Neurosurg , 76, pp.571-577.

Shimokawa, H. et al., 2002. Anti-anginal Effect of Fasudil, a Rho-Kinase Inhibitor, in Patients With Stable Effort Angina: A Multicenter Study. Journal of Cardiovascular Pharmacology, 40(5), pp.751-761.

Sidhu, A. et al., 2004. The role of alpha-synuclein in both neuroprotection and neurodegeneration. Annals of the New York Academy of Sciences, 1035, pp.250-270.

Silver, J. \& Miller, J.H., 2004. Regeneration beyond the glial scar. Nature reviews, 5(2), pp.146-56.

Singleton, A. et al., 2004. Association between cardiac denervation and parkinsonism caused by alpha-synuclein gene triplication. Brain, 127(Pt 4), pp.768-72.

Smith, Y. et al., 2012. Parkinson's disease therapeutics: new developments and challenges since the introduction of levodopa. Neuropsychopharmacology, 37(1), pp.213-46.

Spillantini, M.G. et al., 1997. Alpha-synuclein in Lewy bodies. Nature, 388(6645), pp.839-40.

Spillantini, M.G. et al., 1998. Filamentous alpha-synuclein inclusions link multiple system atrophy with Parkinson's disease and dementia with Lewy bodies. Neuroscience letters, 251(3), pp.205-8.

Stambolic, V. et al., 1998. Negative Regulation of PKB/Akt-Dependent Cell Survival by the Tumor Suppressor PTEN. Cell, 95(1), pp.29-39.

Stefani, A. et al., 2007. Bilateral deep brain stimulation of the pedunculopontine and subthalamic nuclei in severe Parkinson's disease. Brain, 130(Pt 6), pp.1596-607.

Stott, S.R.W. \& Barker, R. a, 2014. Time course of dopamine neuron loss and glial response in the 6-OHDA striatal mouse model of Parkinson's disease. The European journal of neuroscience, 39(6), pp.1042-56.

Su, X. et al., 2009. Synuclein activates microglia in a model of Parkinsons disease. Neurobiology of Aging, 29(11), pp.1690-1701.

Tang, A., Campbell, W. \& Nithipatikom, K., 2013. ROCk1 feedback regulation of the upstream small GTPase RhoA. Cell Signal., 24(7), pp.1375-1380. 
Tatenhorst, L. et al., 2014. Rho Kinase Inhibition by Fasudil in the Striatal 6Hydroxydopamine Lesion Mouse Model of Parkinson Disease. Journal of neuropathology and experimental neurology, 73(8), pp.770-779.

Tenenbaum, L., 2004. Recombinant AAV-mediated gene delivery to the central nervous system. Journal of gene Medicine, pp.212-222.

Tenenbaum, L., Lehtonen, E. \& Monahan, P., 2003. Evaluation of Risks Related to the Use of Adeno-Associated Virus-Based Vectors. Current Gene Therapy, 3(6), pp.545-565.

Tomac, A. et al., 1995. Protection and repair of the nigrostriatal dopaminergic system by GDNF in vivo. Nature, 373, pp.335-9.

Tompkins, M.M. \& Hill, W.D., 1997. Contribution of somal Lewy bodies to neuronal death. Brain Research, 775, pp.24-29.

Tönges, L. et al., 2012. Inhibition of rho kinase enhances survival of dopaminergic neurons and attenuates axonal loss in a mouse model of Parkinson's disease. Brain, 135, pp.3355-70.

Tönges, L. et al., 2014. Rho kinase inhibition modulates microglia activation and improves survival in a model of amyotrophic lateral sclerosis. Glia, 62(2), pp.217-232.

Tönges, L. et al., 2011. ROCKing Regeneration: Rho Kinase Inhibition as Molecular Target for Neurorestoration. Frontiers in molecular neuroscience, 4, p.39.

Totsukawa, G. et al., 2000. Distinct Roles of Rock (Rho-Kinase) and MLCK in Spatial Regulation of MLC Phosphorylation for Assembly of Stress Fibers and Focal Adhesions in 3t3 Fibroblasts. The Journal of Cell Biology, 150(4), pp.797-806.

Ungerstedt, U., 1971. Postsynaptic Supersensitivity after 6-Hydroxy-dopamine Induced Degeneration of the Nigro-striatal Dopamine System. Acta Physiologica Scandinavica, 82(S367), pp.69-93.

Vandeputte, C. et al., 2010. Automated quantitative gait analysis in animal models of movement disorders. BMC Neurosciences. 11:92.

Verma, P. et al., 2005. Axonal protein synthesis and degradation are necessary for efficient growth cone regeneration. The Journal of neuroscience, 25(2), pp.331-42.

Villar-Cheda, B. et al., 2012. Involvement of microglial RhoA/Rho-kinase pathway activation in the dopaminergic neuron death. Role of angiotensin via angiotensin type 1 receptors. Neurobiology of disease, 47(2), pp.268-79.

Volkow, N.D. et al., 1990. Dopamine Transporters Decrease with Age. Journal of Nuclear Medicine. Vol 37. 4:554-559. 
Walsh, S., Finn, D.P. \& Dowd, E., 2011. Time-course of nigrostriatal neurodegeneration and neuroinflammation in the 6-hydroxydopamine-induced axonal and terminal lesion models of Parkinson's disease in the rat. Neuroscience, 175, pp.251-261.

Wilhelm, B.G., Groemer, T.W. \& Rizzoli, S.O., 2010. The same synaptic vesicles drive active and spontaneous release. Nature neuroscience, 13(12), pp.1454-6.

Willig, K.I. et al., 2006. STED microscopy reveals that synaptotagmin remains clustered after synaptic vesicle exocytosis. Nature, 440(7086), pp.935-9.

Winkler, C., Lee, C.S. \& Bjo, A., 1996. Short-Term GDNF Treatment Provides Long-Term Rescue of Lesioned Nigral Dopaminergic Neurons in a Rat Model of Parkinson's Disease. Journal of Neuroscience, 16(22), pp.7206-7215.

Yan, M.H., Wang, X. \& Zhu, X., 2013. Mitochondrial defects and oxidative stress in Alzheimer disease and Parkinson disease. Free radical biology \& medicine, 62, pp.90-101.

Yiu, G. \& Zhigang, H., 2009. Glial inhibition of CNS axon regeneration. Nat Rev Neurosci, 7(8), pp.617-627.

Yu, J.-Z. et al., 2010. Therapeutic potential of experimental autoimmune encephalomyelitis by Fasudil, a Rho kinase inhibitor. Journal of neuroscience research, 88(8), pp.1664-72.

Zecca, L. et al., 2003. Neuromelanin of the substantia nigra: a neuronal black hole with protective and toxic characteristics. Trends in neurosciences, 26(11), pp.578-80.

Zhao, W. et al., 2011. Extracellular mutanz SOD1 induced microglial-mediated motoneuron injury. Glia, 58(2), pp.231-243.

Zhou, Z. et al., 2011. Rho GTPase regulation of $\alpha$-synuclein and VMAT2: Implications for pathogenesis of Parkinson's disease. Molecular and Cellular Neuroscience, 48(1), pp.2937. 


\subsection{Curriculum Vitae}

\section{Personal details}

Name

Kim-Ann Saal

Adress

Rosdorfer Weg 19

37073 Göttingen

Email

kim-ann.saal@med.uni-goettingen.de

Date and place of birth

25th of September 1983 in Bad Harzburg

Nationality

German

\section{Education}

August 2001 until June 2004

Werner-von-Siemens-Gymnasium, Bad Harzburg

June 2004

Abitur

October 2005 until August 2008

Study of biologie (Bachelor) at the University of Bremen

- Bachelor thesis at the Brain research Institute, Department of Behavioral Physiology and Developmental Neurobiology (AG Prof. Dr. Ursula Dicke/ Prof. Dr. Dr. Gerhard Roth) - subject: "Influence of attention and motivation on the prey capturing behavior of the salamander Plethodon shermani"

August 2008

Examination: Bachelor of Science

October 2008 until September 2010

Study of Neurosciences (Master) at the University of Bremen

- Master thesis at the University Medicine Göttingen, Department of Neurology (Prof. Dr. Paul Lingor/ Prof. Dr. Mathias Bähr) subject: "Effect of the Rho-kinase inhibitor Y-27632 on survival and regeneration of primary motoneurons in vitro"

September 2010

January 2011 until

$30^{\text {th }}$ of January 2015
Examination: Master of Science

PhD-Studies at the Georg-August University Göttingen (University Medicine, Department of Neurology) -Subject: "Modulating the ROCK pathway in models of Parkinson's disease" 


\subsection{Publications}

Saal KA, Koch JC, Tatenhorst L, Szegő EM, Ribas VT, Michel U, Bähr M, Tönges L, Lingor P.: AAV.shRNA-mediated downregulation of ROCK2 attenuates degeneration of dopaminergic neurons in toxin-induced models of Parkinson's disease in vitro and in vivo. Neurobiol. Dis. 2014, Oct 2.; 73: 150-162.

Tönges L, Frank T, Tatenhorst L, Saal KA, Koch JC, Szego ÉM, Bähr M, Weishaupt JH, Lingor P.: Inhibition of rho kinase enhances survival of dopaminergic neurons and attenuates axonal loss in a mouse model of Parkinson's disease. Brain. 2012, 135: 3355-70.

Günther R, Saal KA, Suhr M, Scheer D, Koch JC, Bähr M, Lingor P, Tönges L.: The rho kinase inhibitor Y-27632 improves motor performance in male SOD1 (G93A) mice. Front. Neurosci. 2014, Oct. 7; 8(304): 1-9.

Tönges L, Szegö EM, Hause P, Saal KA, Tatenhorst L, Koch JC, D Hedouville Z, Dambeck V, Kügler S, Dohm CP, Bähr M, Lingor P.: Alpha-synuclein mutations impair axonal regeneration in models of Parkinson's disease. Front Aging Neurosci. 2014, Sep. 10; 6(239): 1-10.

Tatenhorst L, Tönges L, Saal KA, Koch JC, Szegő ÉM, Bähr M, Lingor P.: Rho kinase inhibition by fasudil in the striatal 6-hydroxydopamine lesion mouse model of Parkinson disease. J Neuropathol Exp Neurol. 2014, Aug.73 (8):770-9.

Tönges L, Günther R, Suhr M, Jansen J, Balck A, Saal KA, Barski E, Nientied T, Götz AA, Koch JC, Mueller BK, Weishaupt JH, Sereda MW, Hanisch UK, Bähr M, Lingor P.: Rho kinase inhibition modulates microglia activation and improves survival in a model of amyotrophic lateral sclerosis. Glia. 2014, Feb. 62(2):217-32. 


\subsection{Acknowledgements}

Finally it is my pleasure to thank all people, who supported me during the time of my PhD work!

Especially I would like to thank:

Prof. Dr. Paul Lingor for supervising and guiding me during my work;

Prof. Dr. Mathias Bähr for providing me the chance to work in his laboratory and for making this work possible;

Prof. Dr. Silvio Rizzoli for the cooperation and discussions, as well as the members of his laboratory, especially Sven Truckenbrodt and Katharina Kröhnert for all their help;

Prof. Dr. Martin Göpfert for being part of my Thesis Committee and his feedback on my projects during the years;

All members of the Waldweg Lab, especially Lisa Barski and Vivian Dambeck for their excellent technical and amicable support, as well as Anna E. Roser, Lars Tatenhorst, Lars Tönges and all others who supported me during my work and created a pleasant atmosphere in the lab;

My dear parents and sister Katja, who supported me in all ways they could do;

My dear Matze for loving me;

and all my dear friends, who always believed in me and encouraged me also in hard times, as well as drawing my attention also to other things that should be important in life of a PhD student :) 